\title{
BCJ relations from a new symmetry of gauge-theory amplitudes
}

\author{
Robert W. Brown ${ }^{a}$ and Stephen G. Naculich ${ }^{b, c}$ \\ ${ }^{a}$ Department of Physics, Case Western Reserve University, \\ Cleveland, $\mathrm{OH} 44106$, U.S.A. \\ ${ }^{b}$ Department of Physics, Bowdoin College, \\ Brunswick, ME 04011, U.S.A. \\ ${ }^{c}$ Michigan Center for Theoretical Physics (MCTP), \\ Department of Physics, University of Michigan, \\ Ann Arbor, MI 48109, U.S.A. \\ E-mail: rwb@case.edu, naculich@bowdoin.edu
}

ABSTRACT: We introduce a new set of symmetries obeyed by tree-level gauge-theory amplitudes involving at least one gluon. The symmetry acts as a momentum-dependent shift on the color factors of the amplitude. Using the radiation vertex expansion, we prove the invariance under this color-factor shift of the $n$-gluon amplitude, as well as amplitudes involving massless or massive particles in an arbitrary representation of the gauge group with spin zero, one-half, or one. The Bern-Carrasco-Johansson relations are a direct consequence of this symmetry.

We also introduce the cubic vertex expansion of an amplitude, and use it to derive a generalized-gauge-invariant constraint on the kinematic numerators of the amplitude. We show that the amplitudes of the bi-adjoint scalar theory are invariant under the color-factor symmetry, and use this to derive the null eigenvectors of the propagator matrix.

We generalize the color-factor shift to loop level, and prove the invariance under this shift of one-loop $n$-gluon amplitudes in any theory that admits a color-kinematic-dual representation of numerators. We show that the one-loop color-factor symmetry implies known relations among the integrands of one-loop color-ordered amplitudes.

Keywords: Duality in Gauge Field Theories, Gauge Symmetry, Scattering Amplitudes, Space-Time Symmetries

ArXiv EPrint: 1608.04387 


\section{Contents}

1 Introduction 2

2 Color-factor symmetry and its consequences $\quad 6$

$\begin{array}{lll}2.1 & \text { Color-factor shift for } n \text {-gluon amplitudes } & 7\end{array}$

2.2 Fundamental BCJ relations from the color-factor symmetry 9

$\begin{array}{lll}2.3 & \text { Cubic vertex expansion } & 10\end{array}$

2.4 Constraint on kinematic numerators from the color-factor symmetry 11

$\begin{array}{ll}2.5 & \text { Kinematic numerator shift symmetry } \\ & 12\end{array}$

3 Proof of color-factor symmetry for four-gluon amplitudes $\quad 13$

$\begin{array}{ll}3.1 & \text { Attaching a gluon to a leg } \\ 3.2 & 14\end{array}$

$\begin{array}{ll}3.2 & \text { Attaching a gluon to a vertex } \\ & 15\end{array}$

$\begin{array}{lll}3.3 & \text { Kinematic numerators of the four-gluon amplitude } & 15\end{array}$

$\begin{array}{ll}3.4 \text { Kinematic Jacobi relation } & 16\end{array}$

4 Proof of color-factor symmetry for $n$-gluon amplitudes $\quad 18$

$\begin{array}{lll}4.1 & \text { Attaching a gluon to an external leg } & 18\end{array}$

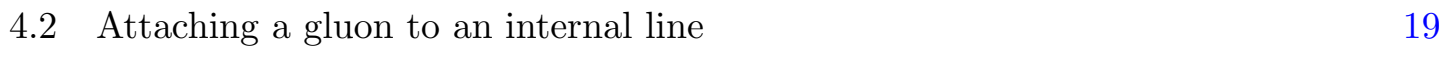

$\begin{array}{ll}4.3 & \text { Radiation vertex expansion } \\ & 20\end{array}$

4.4 Invariance of the radiation vertex expansion under color-factor symmetry 21

5 Color-factor symmetry for more general amplitudes $\quad 23$

6 Proof of color-factor symmetry for the $\mathcal{A}_{4}\left(\bar{\psi}_{1}, \psi_{2}, g_{3}, g_{4}\right)$ amplitude $\quad 26$

6.1 Kinematic numerators for spin-one-half fundamentals 26

6.2 Kinematic numerators for spin-zero fundamentals 27

$\begin{array}{lll}6.3 & \text { Kinematic numerators for spin-one fundamentals } & 29\end{array}$

7 Proof of color-factor symmetry for more general amplitudes $\quad 30$

$\begin{array}{lll}7.1 & \text { Vertices involving spin-one-half fundamentals } & 30\end{array}$

$\begin{array}{lll}7.2 & \text { Vertices involving spin-zero fundamentals } & 32\end{array}$

$\begin{array}{lll}7.3 & \text { Vertices involving spin-one fundamentals } & 36\end{array}$

$\begin{array}{lll}8 & \text { Null eigenvectors of the propagator matrix } & 37\end{array}$

$9 \quad$ Loop-level amplitudes $\quad 39$

9.1 Cubic vertex expansion for loop-level amplitudes 40

9.2 Color-factor symmetry at one-loop level 42

10 Discussion and conclusions $\quad 45$ 


\section{Introduction}

In 2008, Bern, Carrasco, and Johansson discovered a novel set of linear relations satisfied by tree-level color-ordered amplitudes in gauge theories [1]. They arrived at these relations by writing the tree-level $n$-gluon amplitude as a sum over $(2 n-5)$ !! diagrams assembled from cubic vertices

$$
\mathcal{A}_{n}=\sum_{i} \frac{c_{i} n_{i}}{d_{i}}
$$

where the color factor $c_{i}$ associated with the diagram is composed of group theory structure constants $f_{\mathrm{abc}}$, the denominator $d_{i}$ consists of the product of the inverse propagators associated with the diagram, and the kinematic numerator $n_{i}$ depends on the momenta and polarizations of the gluons. All contributions from diagrams with quartic vertices are redistributed among the cubic diagrams. By virtue of the Jacobi identity $f_{\text {abe }} f_{\text {cde }}+f_{\text {ace }} f_{\text {dbe }}+f_{\text {ade }} f_{\text {bce }}=0$ satisfied by the structure constants, the color factors $c_{i}$ obey a set of Jacobi relations of the form

$$
c_{i}+c_{j}+c_{k}=0 .
$$

Because of these linear dependences, the kinematic numerators $n_{i}$ are not uniquely defined, but can undergo generalized gauge transformations $n_{i} \rightarrow n_{i}+\delta n_{i}$ which leave eq. (1.1) unchanged [2,3]. The authors of ref. [1] conjectured that there exists a generalized gauge in which the kinematic numerators satisfy the same algebraic relations as the color factors; in particular, they can be made to satisfy kinematic Jacobi relations

$$
n_{i}+n_{j}+n_{k}=0 .
$$

From this assumption of color-kinematic duality, they demonstrated the existence of new relations (subsequently known as BCJ relations) satisfied by the color-ordered amplitudes $A(1, \cdots, n)$. These relations can be derived from the fundamental BCJ relation (and permutations thereof) $[4-6]$

$$
\sum_{b=3}^{n}\left(\sum_{c=1}^{b-1} k_{2} \cdot k_{c}\right) A(1,3, \cdots, b-1,2, b, \cdots, n)=0
$$

where $k_{a}$ are the (outgoing) momenta of the gluons. Besides color-kinematic duality, these relations rely on the properties of the propagator matrix [7], constructed from the inverse denominators $1 / d_{i}$ (see section 8 for a precise definition). Specifically, as a consequence of momentum conservation, this $(n-2) ! \times(n-2)$ ! matrix has rank $(n-3)$ !, and consequently possesses a set of $(n-3)(n-3)$ ! eigenvectors with eigenvalue zero.

The BCJ relations (1.4) were subsequently proven using string-theory techniques $[4,8]$ and BCFW on-shell recursion [5, 9], providing evidence for the conjecture of tree-level 
color-kinematic duality. Bern et al. conjectured that color-kinematic duality also applies to the integrands of loop-level amplitudes [1,2]; while not proven, this conjecture has been tested for $\mathcal{N}=4$ supersymmetric Yang-Mills theory through four loops [10-18], and for pure Yang-Mills theory through two loops [19, 20]. Another exciting aspect of the story is that gauge-theory kinematic numerators obeying color-kinematic duality can be used to construct gravitational amplitudes via the double copy procedure [1-3]. A recent review of all of these developments may be found in ref. [21].

Despite the fact that the BCJ relations for $n$-gluon amplitudes have been definitively established, interest in tree-level kinematic numerators continues, not least because the numerators that are naturally generated by Feynman rules $^{1}$ generally do not obey the relations (1.3) except in the case of four-point amplitudes [23, 24]. Many approaches have been developed to obtain kinematic numerators that obey color-kinematic duality directly from a Lagrangian approach [3, 25-34].

In this paper, we introduce a new set of symmetries obeyed by tree-level gauge-theory amplitudes, associated with each external gluon in the amplitude. ${ }^{2}$ These symmetries act on the color factors $c_{i}$ of the amplitude, shifting them by momentum-dependent quantities. Since color factors do not carry any momentum dependence, this is a purely formal operation; we prove, however, that the tree-level $n$-gluon amplitude is invariant under these shifts by writing it in an alternative form known as the radiation vertex expansion [35].

We then show that the BCJ relations (1.4) follow as an immediate consequence of the color-factor symmetry of the $n$-gluon amplitude. Although BCJ relations have been previously established, our results reveal a more direct connection to the symmetries of the Lagrangian formulation of gauge theory and its Feynman rules (i.e., gauge and Poincaré invariance) and provide a basis for generalizations.

Let us describe this symmetry in a bit more detail, reserving a full description for section 2. Given a tree-level $n$-gluon color factor $c_{i}$, the choice of one of the external gluon legs $a$ divides the diagram in two at its point of attachment. Let $S_{a, i}$ denote the subset of the remaining legs on one side of this point; it does not matter which side we choose. The shift of the color factor $c_{i}$ associated with gluon $a$ must satisfy

$$
\delta_{a} c_{i} \propto \sum_{c \in S_{a, i}} k_{a} \cdot k_{c} .
$$

Choosing to sum over the complement of $S_{a, i}$ gives the same result (up to sign) due to momentum conservation. The constants of proportionality in eq. (1.5) are then constrained by requiring that the shifted color factors respect all the Jacobi relations satisfied by $c_{i}$ for any values of the momenta.

Consider the case where $a$ is one of the legs involved in the Jacobi identity (see figure 1). Imagine that each of the three graphs in figure 1 is embedded in a larger tree diagram, the same for each. Denote the color factors associated with each diagram by $c_{(r)}$, where $r=1,2,3$. For example, the color factor $c_{(1)}$ for the figure on the left contains

\footnotetext{
${ }^{1}$ String theory can generate numerators that respect color-kinematic duality [22].

${ }^{2}$ For bi-adjoint scalar theories, there is a symmetry for each external massless adjoint scalar in the amplitude.
} 

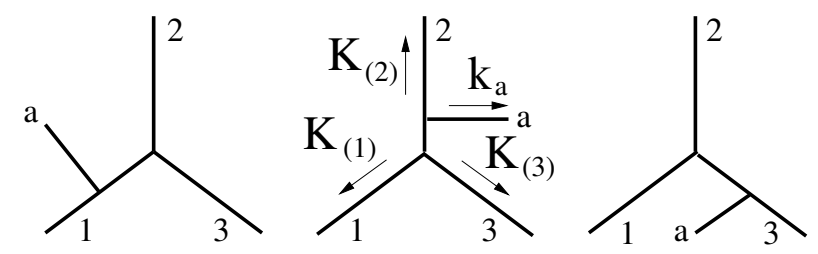

Figure 1. Attaching a gluon to the legs of a cubic vertex. These form parts of the color factors $c_{(1)}, c_{(2)}$, and $c_{(3)}$, respectively.

$\cdots f_{\mathrm{a}_{1} \mathrm{a}_{a} \mathrm{~b}} f_{\mathrm{ba}_{2} \mathrm{~b}_{3}} \cdots$, where the labels on $f_{\mathrm{abc}}$ follow the diagram in clockwise order. Flipping $a$ to the other side of a leg changes the sign of the color factor due to the antisymmetry of $f_{\text {abc }}$. As a result of the Jacobi identity, the color factors obey $\sum_{r=1}^{3} c_{(r)}=0$. By eq. (1.5), the shifts of these color factors are

$$
\delta_{a} c_{(r)}=\alpha_{(r)} k_{a} \cdot K_{(r)}
$$

where $K_{(r)}$ is the momentum flowing out of each leg. Requiring $\sum_{r=1}^{3} \delta_{a} c_{(r)}=0$ implies that $\alpha_{(r)}$ is independent of $r$, as a result of momentum conservation and masslessness of the gluon. A more detailed description of the color-factor shifts is given in section 2 .

The symmetry we have introduced has roots in the radiation symmetry [36] that underlies the general radiation zero theorem $[35,37,38]$. In theories with local gauge couplings and spins $\leq 1$, all single-photon tree amplitudes vanish if the ratios $Q_{c} / k_{a} \cdot k_{c}$ are all equal, ${ }^{3}$ where $k_{a}$ is the photon momentum, and $c$ labels external particles with momentum $k_{c}$ and charge $Q_{c}$. These spin-independent zeros have spin-dependent counterparts where $Q_{c}$ are replaced by numerators $J_{c}$, closely related to the kinematic numerators $n_{i}$ in eq. (1.1). The underlying radiation symmetry refers to invariance under $Q_{c} \rightarrow Q_{c}+\alpha k_{a} \cdot k_{c}$ as well as $J_{c} \rightarrow J_{c}+\beta k_{a} \cdot k_{c}$ for arbitrary $\alpha$ and $\beta$. The extension to nonabelian "charges" has also been considered and the details behind a nonabelian radiation vertex expansion discussed $[35,36]$. The general color-factor symmetry introduced here, however, incorporates crucial nonabelian constraints (Jacobi relations) on $\alpha$, which lead to a complete set of BCJ relations, and have not heretofore been developed. Nevertheless, since the color-factor symmetry relies on the presence of massless gauge bosons, we may regard it as a generalized radiation symmetry.

We also introduce in this paper the cubic vertex expansion of an $n$-point amplitude $\mathcal{A}_{n}$ with respect to one of the gluons $a$. Consider the set of cubic diagrams $I$ that contribute to the $(n-1)$-point amplitude of all the particles in $\mathcal{A}_{n}$ except for gluon $a$. We show that, for any $a$, the amplitude $\mathcal{A}_{n}$ can be written as a triple sum over the legs $r$ of the vertices $v$ of the cubic diagrams $I$ :

$$
\mathcal{A}_{n}=\sum_{I} \sum_{v} \frac{1}{\prod_{s=1}^{3} d_{(a, I, v, s)}} \sum_{r=1}^{3} \frac{c_{(a, I, v, r)} n_{(a, I, v, r)}}{2 k_{a} \cdot K_{(a, I, v, r)}} .
$$

Here $d_{(a, I, v, r)}$ is the product of inverse propagators that branch off from leg $r$ of vertex $v$ of diagram $I, c_{(a, I, v, r)}$ is the color factor of the $n$-point diagram obtained by attaching

\footnotetext{
${ }^{3} \mathrm{~A}$ universal ratio is restrictive and few photon amplitudes have zeros in the physical phase space.
} 
gluon $a$ to leg $r$ of vertex $v$ of diagram $I$ (exactly as in figure 1), and $n_{(a, I, v, r)}$ is the associated $n$-point kinematic numerator. The shift of $c_{(a, I, v, r)}$ associated with gluon $a$ is $\delta_{a} c_{(a, I, v, r)}=\alpha_{(a, I, v)} k_{a} \cdot K_{(a, I, v, r)}$, where, as explained above, $\alpha_{(a, I, v)}$ is independent of $r$. Since the alternative radiation vertex expansion shows that the amplitude $\mathcal{A}_{n}$ is invariant under the color-factor shift, we may conclude from the cubic vertex expansion of $\mathcal{A}_{n}$ that

$$
\sum_{I} \sum_{v} \frac{\alpha_{(a, I, v)}}{\prod_{s=1}^{3} d_{(a, I, v, s)}} \sum_{r=1}^{3} n_{(a, I, v, r)}=0 .
$$

Note that this constraint on the kinematic numerators, less stringent than the kinematic Jacobi relations (which state that $\sum_{r=1}^{3} n_{(a, I, v, r)}=0$ for each vertex), is nonetheless sufficient to imply the BCJ relations (1.4). Moreover, unlike the kinematic Jacobi relations, the condition (1.8) is invariant under generalized gauge transformations. A constraint of precisely the form (1.8) was derived in refs. [39, 40] for the five-gluon amplitude using the monodromy properties of string theory amplitudes.

We show in this paper that more general gauge-theory amplitudes, with both gluons and massless or massive particles in an arbitrary representation of the gauge group and with arbitrary spin $\leq 1$, are also invariant under the color-factor symmetry. Consequently, the kinematic numerators of these amplitudes obey a constraint analogous to eq. (1.8). We further show that color-factor symmetry implies BCJ relations for the color-ordered amplitudes of a class of $n$-point amplitudes involving $n-2$ gluons and a pair of particles in an arbitrary representation of the gauge group and arbitrary spin, as previously conjectured in refs. [41, 42].

BCJ relations for the primitive amplitudes of a more general class of amplitudes containing gluons and an arbitrary number of pairs of differently flavored fundamentals (based on a proper decomposition developed by Melia [43-45] and Johansson and Ochirov [42]) were conjectured by Johansson and Ochirov [42], and subsequently proven using BCFW on-shell recursion by de la Cruz, Kniss, and Weinzierl [46]. In a sequel to this paper [47], we prove that these BCJ relations also follow as a direct consequence of the color-factor symmetry.

The amplitudes of the theory of massless bi-adjoint scalars with cubic interactions [48] also exhibit invariance under color-factor symmetry, as we show using the cubic vertex expansion. In this case, the color-factor shifts are associated with each massless adjoint scalar in the amplitude. As a consequence, we demonstrate the reduced rank of the propagator matrix for the $n$-gluon gauge-theory amplitude by deriving the set of its null eigenvectors.

Finally, we generalize the cubic vertex expansion and color-factor symmetry to looplevel amplitudes containing at least one external gluon. We exhibit an independent set of shifts that act on the color factors of one-loop $n$-gluon amplitudes and which depend on the loop momentum as well as external momenta. These one-loop amplitudes are invariant under color-factor shifts in theories that admit a color-kinematic-dual representation of numerators. The color-factor symmetry also implies certain relations among the integrands of one-loop color-ordered amplitudes that were previously uncovered in refs. [49-51].

The contents of this paper are as follows. In section 2 we define the color-factor shift for the $n$-gluon amplitude and derive the BCJ relations as a consequence of the invariance of 
the amplitude under this shift. We also introduce the cubic vertex expansion, and use it to derive a generalized-gauge-invariant constraint on the kinematic numerators of the $n$-gluon amplitude. We introduce an analogous set of shifts of the kinematic numerators, and show that they correspond to a generalized gauge transformation. In section 3 , we prove the invariance of the four-gluon amplitude under the color-factor symmetry, and in section 4 , we extend this to the $n$-gluon amplitude by using the radiation vertex expansion. In section 5 , we define the color-factor shift for more general amplitudes, and derive the BCJ relations for the class of amplitudes containing $n-2$ gluons and a pair of particles in an arbitrary representation $\mathcal{R}$. In section 6 , we prove the invariance of the four-point amplitude with two gluons and a pair of massive particles of arbitrary spin $\leq 1$ and representation $\mathcal{R}$ under the color-factor symmetry, and in section 7 , we extend this to a general $n$-point amplitude containing gluons and other particles. In section 8 , we prove the invariance of the amplitudes of the bi-adjoint scalar theory under the color factor symmetry, and derive the null eigenvectors of the propagator matrix. In section 9 , we generalize the cubic vertex expansion and color-factor symmetry to loop-level amplitudes, and derive a constraint on the integrands of one-loop color-ordered amplitudes. Section 10 contains a discussion and conclusions. In appendix A, we write the shifts for all the color factors of the five-gluon amplitude, and derive the explicit constraint on the kinematic numerators that follow from the color-factor symmetry.

\section{Color-factor symmetry and its consequences}

We begin this section by introducing the color-factor symmetry in the simplest context, the tree-level four-gluon amplitude

$$
\mathcal{A}_{4}=\frac{c_{s} n_{s}}{s}+\frac{c_{t} n_{t}}{t}+\frac{c_{u} n_{u}}{u}
$$

where

$$
c_{s}=f_{\mathrm{a}_{1} \mathrm{a}_{2} \mathrm{~b}} f_{\mathrm{ba}_{3} \mathrm{~b}_{4}}, \quad c_{t}=f_{\mathrm{a}_{1} \mathrm{a}_{4} \mathrm{~b}} f_{\mathrm{ba}_{2} \mathrm{~b}_{3}}, \quad c_{u}=f_{\mathrm{a}_{1} \mathrm{a}_{3} \mathrm{~b}} f_{\mathrm{ba}_{4} \mathrm{~b}_{1}}
$$

and $s, t$, and $u$ are Mandelstam variables. We define the four-point color-factor shift to act $\operatorname{as}^{4}$

$$
c_{s} \rightarrow c_{s}+\alpha s, \quad c_{t} \rightarrow c_{t}+\alpha t, \quad c_{u} \rightarrow c_{u}+\alpha u
$$

where $\alpha$ is arbitrary. Eq. (2.3) preserves the Jacobi relation $c_{s}+c_{t}+c_{u}=0$ by virtue of momentum conservation $s+t+u=0$.

The statement that eq. (2.1) is invariant under eq. (2.3) implies the kinematic Jacobi relation

$$
n_{s}+n_{t}+n_{u}=0
$$

It is well-known $[23,24]$ that eq. $(2.4)$ is satisfied in the case of the four-gluon amplitude, and we will show this explicitly in section 3 . This serves as proof of the invariance of the four-gluon amplitude under the color-factor shift.

\footnotetext{
${ }^{4}$ In the case of the four-gluon amplitude, the shifts associated with various legs are all the same.
} 


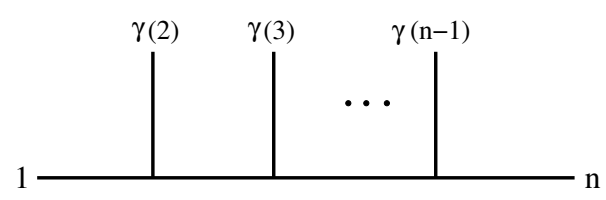

Figure 2. Diagram for the half-ladder color factor $\mathbf{c}_{1 \gamma(2) \cdots \gamma(n-1) n}$.

Recall that the kinematic numerators $n_{i}$ are not uniquely defined by eq. (2.1) because a generalized gauge transformation

$$
n_{s} \rightarrow n_{s}+\beta s, \quad n_{t} \rightarrow n_{t}+\beta t, \quad n_{u} \rightarrow n_{u}+\beta u
$$

(with $\beta$ arbitrary) leaves eq. (2.1) unchanged by virtue of the Jacobi identity $c_{s}+c_{t}+$ $c_{u}=0$. In the case of the four-gluon amplitude, however, the sum $n_{s}+n_{t}+n_{u}$ is welldefined: it is invariant under the generalized gauge transformation (2.5) due to momentum conservation.

The four-gluon amplitude can be written in terms of color-ordered amplitudes as

$$
\mathcal{A}_{4}=c_{s} A(1,2,3,4)-c_{u} A(1,3,2,4) .
$$

Invariance of eq. (2.6) under the shift (2.3) immediately implies

$$
\delta \mathcal{A}_{4}=s A(1,2,3,4)-u A(1,3,2,4)=0
$$

which is the four-gluon BCJ relation [1].

\subsection{Color-factor shift for $n$-gluon amplitudes}

Next we turn to tree-level $n$-gluon amplitudes with $n>4$, which may be written as a sum over diagrams composed of cubic vertices (referred to as cubic diagrams) [1]

$$
\mathcal{A}_{n}=\sum_{i} \frac{c_{i} n_{i}}{d_{i}}
$$

Associated with each cubic diagram $i$ is a color factor $c_{i}$ obtained by sewing together structure constants $f_{\mathrm{abc}}$. Among these color factors $c_{i}$ we may identify the subset of halfladder color factors $\mathbf{c}_{\alpha}$ defined by (see figure 2)

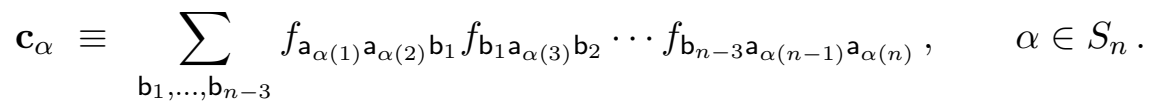

The color factors $c_{i}$ are not independent but obey a set of Jacobi relations. Using the procedure outlined in ref. [52], the Jacobi identity $f_{\text {abe }} f_{\text {cde }}+f_{\text {ace }} f_{\text {dbe }}+f_{\text {ade }} f_{\text {bce }}=0$ may be repeatedly applied to reduce each $c_{i}$ to a linear combination of half-ladder color factors

$$
c_{i}=\sum_{\gamma \in S_{n-2}} M_{i, 1 \gamma n} \mathbf{c}_{1 \gamma n}, \quad \mathbf{c}_{1 \gamma n} \equiv \mathbf{c}_{1 \gamma(2) \cdots \gamma(n-1) n}
$$

where $\gamma$ denotes a permutation of $\{2, \cdots, n-1\}$. The $(n-2)$ ! half-ladders $\mathbf{c}_{1 \gamma n}$ form an independent set. Alternatively, $M_{i, 1 \gamma n}$ may be computed by rewriting $c_{i}$ using $f_{\mathrm{abc}}=$ 

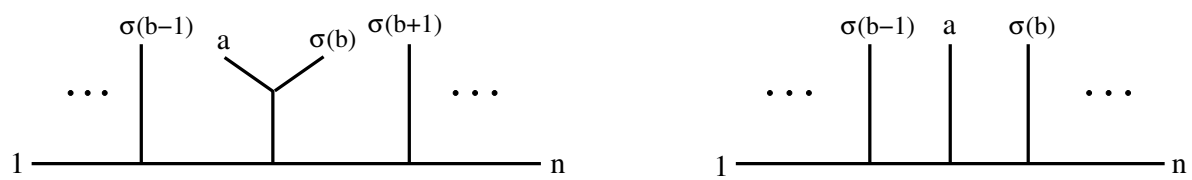

Figure 3. Diagrams with color factors $c_{1 \cdots \sigma(b-1)[a \sigma(b)] \sigma(b+1) \cdots n}$ and $\mathbf{c}_{1 \cdots \sigma(b-1) a \sigma(b) \cdots n}$.

$\operatorname{Tr}\left(\left[T^{\mathrm{a}}, T^{\mathrm{b}}\right] T^{\mathrm{c}}\right)$, reducing the resulting expression to a linear combination of single traces, and then identifying the coefficient of $\operatorname{Tr}\left(T^{\mathrm{a}_{1}} T^{\mathrm{a}_{\gamma(2)}} \cdots T^{\mathrm{a}_{\gamma(n-1)}} T^{\mathrm{a}_{n}}\right)$ (see e.g., ref. [53]).

We now define a set of momentum-dependent shifts, associated with each external gluon $a$ in the amplitude, that act on the color factors $c_{i}$. The action of the shift $\delta_{a} c_{i}$ associated with gluon $a$ is constrained by two requirements: (I) that it preserve all the Jacobi relations satisfied by $c_{i}$, and (II) that it satisfy

$$
\delta_{a} c_{i} \propto \sum_{c \in S_{a, i}} k_{a} \cdot k_{c}
$$

where $S_{a, i}$ denotes the subset of the external particles on one side ${ }^{5}$ of the point at which $a$ is attached to $c_{i}$. In particular, if $c_{i}$ is a color factor in which gluon $a$ is attached to an external leg $b$, the shift is proportional to $k_{a} \cdot k_{b}$, which is an inverse propagator in the associated Feynman diagram. More generally, eq. (2.11) is related (see eq. (2.20)) to the propagators in the Feynman diagram associated with $c_{i}$.

Consider the subset of $n$-point color factors obtained from a given $(n-1)$-point cubic diagram $I$ by attaching gluon $a$ to it in all possible ways. One of these color factors has gluon $a$ attached to external leg 1 of the $(n-1)$-point diagram $;{ }^{6}$ define its shift to be $\alpha_{I} k_{a} \cdot k_{1}$. One may easily verify (using the argument in the introduction) that the conditions (I) and (II) above uniquely fix the coefficients of the shifts of all the other color factors in this subset. The coefficients $\alpha_{I}$ for different $(n-1)$-point diagrams are then constrained by Jacobi relations among their color factors.

We now demonstrate that there is an $(n-3)$ !-parameter family of color-factor shifts associated with each gluon $a$ in the $n$-gluon amplitude. First choose $a \in\{2, \cdots, n-1\}$, and consider the subset of half-ladder color factors $\mathbf{c}_{1 a \sigma n}$, where $\sigma \in S_{n-3}$ denotes a permutation of $\{2, \cdots, n-1\} \backslash\{a\}$. We define the color-factor shift associated with gluon $a$ to act on these half ladders as

$$
\delta_{a} \mathbf{c}_{1 a \sigma(2) \cdots \sigma(n-1) n}=\alpha_{a, \sigma} k_{a} \cdot k_{1}
$$

where $\alpha_{a, \sigma}$ are a set of $(n-3)$ ! arbitrary, independent constants (or functions) for each $a$. Let $c_{1 \cdots \sigma(b-1)[a \sigma(b)] \sigma(b+1) \cdots n}$ denote the color factor shown in figure 3 ; its shift is proportional to $k_{a} \cdot k_{\sigma(b)}$. This together with eq. (2.12) and the Jacobi relation

$$
c_{1 \sigma(2) \cdots \sigma(b-1)[a \sigma(b)] \sigma(b+1) \cdots \sigma(n-1) n}=\mathbf{c}_{1 \sigma(2) \cdots \sigma(b-1) a \sigma(b) \cdots \sigma(n-1) n}-\mathbf{c}_{1 \sigma(2) \cdots \sigma(b) a \sigma(b+1) \cdots \sigma(n-1) n}
$$

\footnotetext{
${ }^{5}$ Either side gives the same result up to sign due to momentum conservation.

${ }^{6}$ Unless $a=1$.
} 
implies that $\delta_{a}$ acts on the independent half-ladder color factors $\mathbf{c}_{1 \gamma n}$ as $^{7}$

$$
\delta_{a} \mathbf{c}_{1 \sigma(2) \cdots \sigma(b-1) a \sigma(b) \cdots \sigma(n-1) n}=\alpha_{a, \sigma}\left(k_{a} \cdot k_{1}+\sum_{c=2}^{b-1} k_{a} \cdot k_{\sigma(c)}\right), \quad a, b \in\{2, \cdots, n-1\}, \quad b \neq a
$$

consistent with eq. (2.11). The action on the remaining color factors is given by

$$
\delta_{a} c_{i}=\sum_{\gamma \in S_{n-2}} M_{i, 1 \gamma n} \delta_{a} \mathbf{c}_{1 \gamma n}
$$

which is also consistent with eq. (2.11), as may be shown using the procedure described in ref. [52].

The color-factor shifts associated with the gluons $\{2, \cdots, n-1\}$ are not all independent. In particular, the $(n-3)$ !-parameter family of shifts associated with gluon $n-1$ are linear combinations of shifts associated with $a \in\{2, \cdots, n-2\}$ as a result of momentum conservation. $^{8}$ We may also define $(n-3)$ !-parameter families of shifts associated with gluons 1 and $n$. These are also not independent of the others. Thus the dimension of the (abelian) group of color-factor shifts is $(n-3)(n-3)$ !.

\subsection{Fundamental BCJ relations from the color-factor symmetry}

By using eq. (2.10), the tree-level $n$-gluon amplitude (2.8) may be rewritten in the Del Duca-Dixon-Maltoni half-ladder decomposition [52, 54]

$$
\mathcal{A}_{n}=\sum_{\gamma \in S_{n-2}} \mathbf{c}_{1 \gamma n} A(1, \gamma(2), \cdots, \gamma(n-1), n)
$$

where the coefficients

$$
A(1, \gamma(2), \cdots, \gamma(n-1), n)=\sum_{i} \frac{M_{i, 1 \gamma n} n_{i}}{d_{i}}
$$

are color-ordered amplitudes belonging to the Kleiss-Kuijf basis [55]. In the previous subsection, we defined the action of the color-factor symmetry associated with a given gluon $a$. The variation of eq. (2.16) under the shift associated with $a=2$ gives

$$
\delta_{2} \mathcal{A}_{n}=\sum_{\sigma \in S_{n-3}} \alpha_{2, \sigma} \sum_{b=3}^{n}\left(k_{1} \cdot k_{2}+\sum_{c=3}^{b-1} k_{2} \cdot k_{\sigma(c)}\right) A(1, \sigma(3), \cdots, \sigma(b-1), 2, \sigma(b), \cdots, \sigma(n-1), n) .
$$

In sections 3 and 4 , we prove that $\mathcal{A}_{n}$ is invariant under this shift. Since $\alpha_{2, \sigma}$ are arbitrary and independent, this implies that

$$
\sum_{b=3}^{n}\left(k_{1} \cdot k_{2}+\sum_{c=3}^{b-1} k_{2} \cdot k_{\sigma(c)}\right) A(1, \sigma(3), \cdots, \sigma(b-1), 2, \sigma(b), \cdots, \sigma(n-1), n)=0
$$

\footnotetext{
${ }^{7}$ In the case $a=2$, replace $\sigma(2)$ with $\sigma(3)$, and the sum over $c$ should begin with 3 . In the case $a=n-1$, replace $\sigma(n-1)$ with $\sigma(n-2)$.

${ }^{8}$ We have verified this numerically through $n=9$, but we know the result must be true for all $n$ because, as we will see in section 8 , the color-factor shifts correspond to null eigenvectors of the propagator matrix. Since the $(n-2) ! \times(n-2)$ ! propagator matrix has rank $(n-3)$ ! [48] there are at most $(n-3)(n-3)$ ! independent null eigenvectors.
} 
which is the fundamental BCJ relation (1.4). All other permutations of this relation can be obtained using the invariance of the amplitude under the color-factor shifts associated with gluons 3 through $n-1$.

It is known [1] that the BCJ relations reduce the number of independent color-ordered amplitudes from $(n-2)$ ! to $(n-3)$ !. Not surprisingly, the difference between these, $(n-$ $3)(n-3)$ !, is the dimension of the group of color-factor shifts that leave the amplitude invariant.

\subsection{Cubic vertex expansion}

In order to examine the implications of the color-factor symmetry for the kinematic numerators $n_{i}$ appearing in the cubic decomposition (2.8), we introduce in this section the cubic vertex expansion of the amplitude with respect to one of the gluons. This expansion is similar to, but distinct from, the radiation vertex expansion [35] that will be used in sections 4 and 7 to prove the invariance of $n$-point gauge-theory amplitudes under color-factor shifts.

The cubic decomposition (2.8) is a sum over the $(2 n-5)$ !! cubic diagrams of an $n$-gluon amplitude, but for any $a \in\{1, \cdots, n\}$ it can be viewed as a sum over the $(2 n-7) !$ ! cubic diagrams of an $(n-1)$-point function with external legs $\{1, \cdots, n\} \backslash\{a\}$, to each of which gluon $a$ is attached in $2 n-5$ different ways. Let us label these $(n-1)$-point cubic diagrams by $I$ and their denominators by $d_{(a, I)}$.

Each $(n-1)$-point cubic diagram $I$ has $n-3$ vertices, the set of which we denote by $V_{(a, I)}$. For each vertex $v \in V_{(a, I)}$, we can break $d_{(a, I)}$ into three factors $\prod_{r=1}^{3} d_{(a, I, v, r)}$, where $d_{(a, I, v, r)}$ is the product of propagators that branch off from leg $r$ of the vertex. If leg $r$ is an external leg of the diagram, then $d_{(a, I, v, r)}=1$.

We can attach gluon $a$ either to one of the $n-1$ external legs or to one of the $n-4$ internal lines of $I$, yielding altogether $2 n-5$ of the terms in the sum (2.8). Let $K$ be the momentum running through one of the internal lines of $I$. Attaching gluon $a$ to this line will replace the factor $K^{2}$ in $d_{(a, I)}$ with $K^{2}\left(K+k_{a}\right)^{2}$. We split the inverse denominator into two terms using the identity

$$
\frac{1}{K^{2}\left(K+k_{a}\right)^{2}}=\frac{1}{K^{2}\left(2 k_{a} \cdot K\right)}+\frac{1}{\left(-2 k_{a} \cdot K\right)\left(K+k_{a}\right)^{2}}
$$

and we associate each of the terms on the right hand side of the equation with one of the two vertices to which the internal line is connected. Thus, with this doubling of internal line terms, we now have a total of $(n-1)+2(n-4)=3(n-3)$ terms for each $I$; namely, one term for each of the legs of each of the $n-3$ vertices of $I$. We label this term by $(a, I, v, r)$, and write the cubic vertex expansion of the $n$-gluon amplitude with respect to gluon $a$ as

$$
\mathcal{A}_{n}=\sum_{I} \sum_{v \in V_{(a, I)}} \frac{1}{\prod_{s=1}^{3} d_{(a, I, v, s)}} \sum_{r=1}^{3} \frac{c_{(a, I, v, r)} n_{(a, I, v, r)}}{2 k_{a} \cdot K_{(a, I, v, r)}}
$$

where $c_{(a, I, v, r)}$ are the color factors $c_{(r)}$ in figure 1 associated with each vertex $(a, I, v)$, $n_{(a, I, v, r)}$ are the associated kinematic numerators, and $K_{(a, I, v, r)}$ denotes the momentum flowing out of leg $r$. The $c_{(a, I, v, r)}$ and $n_{(a, I, v, r)}$ are equal to the $c_{i}$ and $n_{i}$ in eq. (2.8) up to 
signs (such that $\left.c_{(a, I, v, r)} n_{(a, I, v, r)}=c_{i} n_{i}\right)$. An explicit example of the cubic vertex expansion for the five-gluon amplitude is given in appendix $\mathrm{A}$.

The \pm freedom in the definition of $c_{(a, I, v, r)}$ is used to make the relative signs in the Jacobi relation positive:

$$
\sum_{r=1}^{3} c_{(a, I, v, r)}=0 .
$$

The denominators in each triple also sum to zero

$$
\sum_{r=1}^{3} k_{a} \cdot K_{(a, I, v, r)}=0
$$

by momentum conservation $k_{a}+\sum_{r=1}^{3} K_{(a, I, v, r)}=0$ and the masslessness of the gluon $k_{a}^{2}=0$. A priori, however, there is no reason for the kinematic numerators $n_{(a, I, v, r)}$ associated with each vertex to sum to zero. We will see in the next subsection, however, that the color-factor symmetry of the amplitude leads to a constraint on the sum of kinematic numerators.

\subsection{Constraint on kinematic numerators from the color-factor symmetry}

Having introduced the cubic vertex expansion of the $n$-gluon amplitude with respect to gluon $a$, we now consider the effect of a color-factor shift $\delta_{a}$ on the amplitude. The shift associated with gluon $a$ acts on the color factors appearing in the cubic vertex expansion $(2.21)$ as

$$
\delta_{a} c_{(a, I, v, r)}=\alpha_{(a, I, v)} k_{a} \cdot K_{(a, I, v, r)}
$$

where $\alpha_{(a, I, v)}$ is a linear combination of $\alpha_{a, \sigma}$ uniquely determined by the Jacobi relations. These shifts respect eq. (2.22) by virtue of eq. (2.23). The variation of eq. (2.21) under this shift gives

$$
\delta_{a} \mathcal{A}_{n}=\frac{1}{2} \sum_{I} \sum_{v \in V_{(a, I)}} \frac{\alpha_{(a, I, v)}}{\prod_{s=1}^{3} d_{(a, I, v, s)}} \sum_{r=1}^{3} n_{(a, I, v, r)} .
$$

We prove that $\delta_{a} \mathcal{A}_{n}=0$ in sections 3 and 4 ; hence the color-factor symmetry implies the following constraint on the kinematic numerators

$$
\sum_{I} \sum_{v \in V_{(a, I)}} \frac{\alpha_{(a, I, v)}}{\prod_{s=1}^{3} d_{(a, I, v, s)}} \sum_{r=1}^{3} n_{(a, I, v, r)}=0 .
$$

Now if the constants $\alpha_{(a, I, v)}$ were all independent, then we could conclude from this argument that $\sum_{r=1}^{3} n_{(a, I, v, r)}=0$, i.e. that the kinematic numerators necessarily obey Jacobi relations. This would be in conflict with the well-known fact that the kinematic numerators obtained from Feynman rules in general do not satisfy color-kinematic duality ${ }^{9}{ }^{\text {indeed }}$ this is not a valid inference from eq. (2.26) because the $\alpha_{(a, I, v)}$ are not independent. The set of $\alpha_{(a, I, v)}$ for all the vertices of a given diagram $I$ are equal (up to signs) because any

\footnotetext{
${ }^{9}$ Only in the case $n=4$, where there is only one term in the sum (2.26), may we conclude that $n_{s}+n_{t}+n_{u}=0$.
} 
two adjacent vertices share a common color factor (see the example discussed in the appendix). In fact, with an appropriate choice of signs for $c_{(a, I, v, r)}$, the $\alpha_{(a, I, v)}$ may be made independent of $v$. The $\alpha_{(a, I)}$ for different diagrams $I$ are further constrained by the Jacobi relations among the color factors of $I$.

While eq. (2.26) does not imply that the numerators satisfy the kinematic Jacobi relations, it does impose a set of (generalized-gauge-invariant) conditions that the colorkinematic violations $\Delta_{i j k}=n_{i}+n_{j}+n_{k}$ must satisfy. We wish to emphasize that, while the kinematic Jacobi relations $n_{i}+n_{j}+n_{k}=0$ are not invariant under generalized gauge transformations $^{10}$ (hence the actual claim of color-kinematic duality is that there exists a generalized gauge in which they hold true), the conditions (2.26) are invariant under generalized gauge transformations. The argument for this is simple. A generalized gauge transformation is a transformation $n_{i} \rightarrow n_{i}^{\prime}$ that leaves the amplitude (2.8) unchanged. Hence by starting with $\mathcal{A}_{n}=\sum_{i}\left(c_{i} n_{i}^{\prime} / d_{i}\right)$ and following the steps above (since the condition $\delta_{a} \mathcal{A}_{n}=0$ is also gauge invariant), we obtain the same result (2.26) except with $n_{(a, I, v, r)}$ replaced with $n_{(a, I, v, r)}^{\prime}$.

To obtain a more explicit form of eq. (2.26), we would need to identify all the linear dependences among the $\alpha_{(a, I, v)}$ required by the color Jacobi relations. Previously, we observed that the number of independent color-factor shifts was $(n-3)(n-3)$ !, parametrized by constants $\alpha_{a, \sigma}$, where $a=2, \cdots, n-2$ and $\sigma \in S_{n-3}$ denotes a permutation of $\{2, \cdots, n-$ $1\} \backslash\{a\}$. If we were to write $\alpha_{(a, I, v)}$ in terms of these independent constants, eq. (2.26) would yield $(n-3)(n-3)$ ! independent constraints on the $\Delta_{i j k}$. In appendix A, we carry out this procedure for the five-gluon amplitude.

While the BCJ relations (2.19) were originally derived as a consequence of the assumption of color-kinematic duality, it was known $[39,40]$ from early on that they are equivalent to a set of weaker conditions on the numerators. The conditions for five-gluon numerators were derived in refs. $[39,40]$ as a consequence of the monodromy properties of string-theory amplitudes. These conditions are equivalent to eq. (2.26), as we show in appendix A. In this section, we have demonstrated that both eq. (2.26) and the BCJ relations (2.19) are a consequence of the invariance of the amplitude under the color-factor symmetry.

\subsection{Kinematic numerator shift symmetry}

We have considered the effect on the amplitude of a shift of the color factors. One may ask what effect an analogous shift of the kinematic numerators would have. ${ }^{11}$ We show in this section that such a shift is simply a generalized gauge transformation.

Let us define the kinematic shift associated with leg $a$, where $a \in\{2, \cdots, n-1\}$, on the half-ladder numerator $\mathbf{n}_{1 \gamma n}$ to be

$$
\delta_{a} \mathbf{n}_{1 \sigma(2) \cdots \sigma(b-1) a \sigma(b) \cdots \sigma(n-1) n}=\beta_{a, \sigma}\left(k_{1} \cdot k_{a}+\sum_{c=2}^{b-1} k_{a} \cdot k_{\sigma(c)}\right), \quad a, b \in\{2, \cdots, n-1\}, \quad b \neq a
$$

\footnotetext{
${ }^{10}$ Again except in the case of four-gluon amplitudes.

${ }^{11}$ These can be considered a generalization of the shifts of $J$ considered in refs. [36, 38].
} 
where $\sigma \in S_{n-3}$ denotes a permutation of $\{2, \cdots, n-1\} \backslash\{a\}$, and $\beta_{a, \sigma}$ are a set of arbitrary constants (or functions). The action on all other numerators $n_{i}$ is then defined by

$$
\delta_{a} n_{i}=\sum_{\gamma \in S_{n-2}} M_{i, 1 \gamma n} \delta_{a} \mathbf{n}_{1 \gamma n}
$$

Note that we have not assumed that the $n_{i}$ obey the Jacobi relations (1.3). However, the numerator shifts defined by eq. (2.27) and (2.28) will satisfy

$$
\delta_{a}\left(n_{i}+n_{j}+n_{k}\right)=0
$$

so if the $n_{i}$ do satisfy kinematic Jacobi relations, the shifted numerators will continue to do so, and if they do not, then the neither will the shifted numerators.

Now consider the cubic vertex expansion of the $n$-gluon amplitude

$$
\mathcal{A}_{n}=\sum_{I} \sum_{v \in V_{(a, I)}} \frac{1}{\prod_{s=1}^{3} d_{(a, I, v, s)}} \sum_{r=1}^{3} \frac{c_{(a, I, v, r)} n_{(a, I, v, r)}}{2 k_{a} \cdot K_{(a, I, v, r)}} .
$$

The kinematic shift with respect to gluon $a$ acts on the numerators appearing in this expression as

$$
\delta_{a} n_{(a, I, v, r)}=\beta_{(a, I, v)} k_{a} \cdot K_{(a, I, v, r)}
$$

and therefore on the amplitude itself as

$$
\delta_{a} \mathcal{A}_{n}=\frac{1}{2} \sum_{I} \sum_{v \in V_{(a, I)}} \frac{\beta_{(a, I, v)}}{\prod_{s=1}^{3} d_{(a, I, v, s)}} \sum_{r=1}^{3} c_{(a, I, v, r)} .
$$

This vanishes courtesy of eq. (2.22); hence the $n$-gluon amplitude is invariant under the shift of kinematic numerators. This is precisely the definition of a generalized gauge transformation $[2,3]$.

\section{Proof of color-factor symmetry for four-gluon amplitudes}

In this section we prove that the tree-level four-gluon amplitude is invariant under the color-factor symmetry. In doing so, we develop some results that will be necessary for our more general proof of the invariance of the $n$-gluon amplitude in the next section.

The four-gluon amplitude can be constructed from a three-gluon vertex by attaching a fourth gluon to a propagator emanating from each of the legs of the vertex or to the vertex itself. This yields

$$
\mathcal{A}_{4}=\sum_{r=1}^{3} \frac{c_{(r)} n_{(r)}}{\left(k_{r}+k_{4}\right)^{2}}
$$

where

$$
c_{(1)}=f_{\mathrm{a}_{1} \mathrm{a}_{4} \mathrm{~b}} f_{\mathrm{ba}_{2} \mathrm{a}_{3}}, \quad c_{(2)}=f_{\mathrm{a}_{2} \mathrm{a}_{4} \mathrm{~b}} f_{\mathrm{ba}_{3} \mathrm{a}_{1}}, \quad c_{(3)}=f_{\mathrm{a}_{3} \mathrm{a}_{4} \mathrm{~b}} f_{\mathrm{ba}_{1} \mathrm{a}_{2}} .
$$


The kinematic numerator $n_{(r)}$ receives contributions from the diagram in which gluon 4 is attached to leg $r$ as well as from the four-gluon vertex. The color-factor symmetry (associated with gluon 4) acts on the color factors (3.2) and the four-gluon amplitude (3.1) as

$$
\delta_{4} c_{(r)}=\alpha_{4} k_{4} \cdot k_{r} \quad \Longrightarrow \quad \delta_{4} \mathcal{A}_{4}=\frac{1}{2} \alpha_{4} \sum_{r=1}^{3} n_{(r)} .
$$

Thus, by showing that $\sum_{r=1}^{3} n_{(r)}=0$, we will establish that $\delta_{4} \mathcal{A}_{4}=0$. This we now proceed to do.

\subsection{Attaching a gluon to a leg}

The three-gluon vertex is ${ }^{12}$

$$
-\frac{i g}{\sqrt{2}} f_{\mathrm{a}_{1} \mathrm{a}_{2} \mathrm{a}_{3}} V^{\mu_{1} \mu_{2} \mu_{3}}\left(k_{1}, k_{2}, k_{3}\right)
$$

where

$$
V^{\mu_{1} \mu_{2} \mu_{3}}\left(k_{1}, k_{2}, k_{3}\right)=\eta^{\mu_{1} \mu_{2}}\left(k_{2}-k_{1}\right)^{\mu_{3}}+\eta^{\mu_{2} \mu_{3}}\left(k_{3}-k_{2}\right)^{\mu_{1}}+\eta^{\mu_{3} \mu_{1}}\left(k_{1}-k_{3}\right)^{\mu_{2}}
$$

and $k_{a}$ are outgoing momenta. In Feynman gauge, the gluon propagator is $-i \eta_{\mu \nu} \delta_{\mathrm{ab}} / k^{2}$. Attaching gluon 4 to leg 1 yields the expression

$$
\begin{aligned}
& \frac{i g^{2}}{2} \frac{f_{\mathrm{a}_{1} \mathrm{a}_{4} \mathrm{~b}} f_{\mathrm{ba}_{2} \mathrm{a}_{3}}}{\left(k_{1}+k_{4}\right)^{2}} V^{\mu_{1} \mu_{4} \nu}\left(k_{1}, k_{4},-k_{1}-k_{4}\right) V_{\nu}^{\mu_{2} \mu_{3}}\left(k_{1}+k_{4}, k_{2}, k_{3}\right) \\
& =\frac{i g^{2}}{2} \frac{c_{(1)}}{\left(k_{1}+k_{4}\right)^{2}}\left[\eta^{\mu_{1} \nu} k_{4}^{\mu_{4}}-\eta^{\mu_{4} \nu} k_{1}^{\mu_{1}}-\eta^{\mu_{1} \mu_{4}}\left(k_{1}+k_{4}\right)^{\nu}\right. \\
& \left.+2 \eta^{\mu_{1} \nu} k_{1}^{\mu_{4}}+2\left(\eta^{\mu_{1} \mu_{4}} k_{4}^{\nu}-\eta^{\mu_{4} \nu} k_{4}^{\mu_{1}}\right)\right] V_{\nu}^{\mu_{2} \mu_{3}}\left(k_{1}+k_{4}, k_{2}, k_{3}\right) .
\end{aligned}
$$

The contribution of this diagram to the four-gluon amplitude (3.1) is obtained by contracting with $\prod_{a=1}^{4} \varepsilon_{a \mu_{a}}$ and dividing by $i$. The first and second terms in the square brackets vanish using $\varepsilon_{a} \cdot k_{a}=0$. The third term vanishes due to

$$
\left(k_{1}+k_{4}\right)^{\nu} V_{\nu}^{\mu_{2} \mu_{3}}\left(k_{1}+k_{4}, k_{2}, k_{3}\right) \varepsilon_{2 \mu_{2}} \varepsilon_{3 \mu_{3}}=0
$$

using $k_{2}^{2}=k_{3}^{2}=0$. The contribution of the remaining terms of eq. (3.6) to the kinematic numerator $n_{(1)}$ can be written

$$
\left.n_{(1)}\right|_{\text {leg }}=g^{2} \varepsilon_{1 \mu_{1}}\left[\varepsilon_{4} \cdot k_{1} \delta_{\nu}^{\mu_{1}}-i \varepsilon_{4 \alpha} k_{4 \beta}\left(S_{1}^{\alpha \beta}\right)_{\nu}^{\mu_{1}}\right] V^{\nu \mu_{2} \mu_{3}}\left(k_{1}+k_{4}, k_{2}, k_{3}\right) \varepsilon_{2 \mu_{2}} \varepsilon_{3 \mu_{3}}
$$

where

$$
\left(S_{r}^{\alpha \beta}\right)^{\mu_{r}}=i\left(\eta^{\alpha \mu_{r}} \delta^{\beta}{ }_{\nu}-\eta^{\beta \mu_{r}} \delta_{\nu}^{\alpha}\right)
$$

are the spin-one angular momentum matrices acting on gluon $r$. These satisfy the Lorentz algebra commutation relations

$$
\left[S_{r}^{\alpha \beta}, S_{r}^{\gamma \delta}\right]=-i\left[\eta^{\alpha \gamma} S_{r}^{\beta \delta}-\eta^{\alpha \delta} S_{r}^{\beta \gamma}-\eta^{\beta \gamma} S_{r}^{\alpha \delta}+\eta^{\beta \delta} S_{r}^{\alpha \gamma}\right]
$$

\footnotetext{
${ }^{12}$ Our structure constants are normalized by $f_{\mathrm{abc}}=\operatorname{Tr}\left(\left[T^{\mathrm{a}}, T^{\mathrm{b}}\right] T^{\mathrm{c}}\right)$ with $\operatorname{Tr}\left(T^{\mathrm{a}} T^{\mathrm{b}}\right)=\delta^{\mathrm{ab}}$, so that $\left[T^{\mathrm{a}}, T^{\mathrm{b}}\right]=$ $f_{\mathrm{abc}} T^{\mathrm{c}}$. This differs from the standard textbook convention by a factor of $i \sqrt{2}$. We use $\eta_{00}=1$ in this paper.
} 


\subsection{Attaching a gluon to a vertex}

Attaching gluon 4 directly to the three-gluon vertex, we obtain the four-gluon vertex

$$
\begin{aligned}
V_{\mathrm{a}_{1} \mathrm{a}_{2} \mathrm{a}_{3} \mathrm{a}_{4}}^{\mu_{1} \mu_{2} \mu_{3} \mu_{4}}=\frac{i g^{2}}{2}[ & f_{\mathrm{a}_{1} \mathrm{a}_{4} \mathrm{~b}} f_{\mathrm{ba}_{2} \mathrm{a}_{3}}\left(\eta^{\mu_{1} \mu_{2}} \eta^{\mu_{3} \mu_{4}}-\eta^{\mu_{1} \mu_{3}} \eta^{\mu_{2} \mu_{4}}\right) \\
& +f_{\mathrm{a}_{2} \mathrm{a}_{4} \mathrm{~b}} f_{\mathrm{ba}_{3} \mathrm{a}_{1}}\left(\eta^{\mu_{2} \mu_{3}} \eta^{\mu_{1} \mu_{4}}-\eta^{\mu_{1} \mu_{2}} \eta^{\mu_{3} \mu_{4}}\right) \\
& \left.+f_{\mathrm{a}_{3} \mathrm{a}_{4} \mathrm{~b}} f_{\mathrm{ba}_{1} \mathrm{a}_{2}}\left(\eta^{\mu_{1} \mu_{3}} \eta^{\mu_{2} \mu_{4}}-\eta^{\mu_{2} \mu_{3}} \eta^{\mu_{1} \mu_{4}}\right)\right]
\end{aligned}
$$

Using eq. (3.5), this can be recast as

$$
V_{\mathrm{a}_{1} \mathrm{a}_{2} \mathrm{a}_{3} \mathrm{a}_{4}}^{\mu_{1} \mu_{2} \mu_{4} \mu_{4}}=-\frac{i g^{2}}{2}\left(c_{(1)} \frac{\partial}{\partial k_{1 \mu_{4}}}+c_{(2)} \frac{\partial}{\partial k_{2 \mu_{4}}}+c_{(3)} \frac{\partial}{\partial k_{3 \mu_{4}}}\right) V^{\mu_{1} \mu_{2} \mu_{3}}\left(k_{1}, k_{2}, k_{3}\right) .
$$

The contribution of this vertex to the four-gluon amplitude is obtained by contracting with $\prod_{a=1}^{4} \varepsilon_{a \mu_{a}}$ and dividing by $i$. This contribution is then parceled out among the three terms in eq. (3.1); the contribution to $n_{(1)}$ is

$$
\left.n_{(1)}\right|_{\text {vertex }}=-g^{2} k_{4} \cdot k_{1} \varepsilon_{4 \mu_{4}} \frac{\partial}{\partial k_{1 \mu_{4}}} V^{\mu_{1} \mu_{2} \mu_{3}}\left(k_{1}, k_{2}, k_{3}\right) \varepsilon_{1 \mu_{1}} \varepsilon_{2 \mu_{2}} \varepsilon_{3 \mu_{3}} .
$$

The reader may be concerned about the use of eq. (3.12) for the following reason. The three-gluon vertex (3.5) can be rewritten using momentum conservation $\sum_{a=1}^{3} k_{a}=0$. For example, we can eliminate $k_{3}$ from eq. (3.5), writing it as

$$
V^{\mu_{1} \mu_{2} \mu_{3}}\left(k_{1}, k_{2}, k_{3}\right)=\eta^{\mu_{1} \mu_{2}}\left(k_{2}-k_{1}\right)^{\mu_{3}}+\eta^{\mu_{2} \mu_{3}}\left(-k_{1}-2 k_{2}\right)^{\mu_{1}}+\eta^{\mu_{3} \mu_{1}}\left(2 k_{1}+k_{2}\right)^{\mu_{2}} .
$$

The partial derivatives $\left(\partial / \partial k_{a \mu_{4}}\right) V\left(k_{1}, k_{2}, k_{3}\right)$ obtained from eq. (3.14) differ from those obtained from eq. (3.5), so that eq. (3.12) gives

$$
\begin{aligned}
V_{\mathrm{a}_{1} \mathrm{a}_{2} \mathrm{a}_{3} \mathrm{a}_{4}}^{\mu_{3} \mu_{4} \mu_{4}}=\frac{i g^{2}}{2}[ & f_{\mathrm{a}_{1} \mathrm{a}_{4} \mathrm{~b}} f_{\mathrm{ba}_{2} \mathrm{a}_{3}}\left(\eta^{\mu_{1} \mu_{2}} \eta^{\mu_{3} \mu_{4}}+\eta^{\mu_{2} \mu_{3}} \eta^{\mu_{1} \mu_{4}}-2 \eta^{\mu_{1} \mu_{3}} \eta^{\mu_{2} \mu_{4}}\right) \\
& \left.+f_{\mathrm{a}_{2} \mathrm{a}_{4} \mathrm{~b}} f_{\mathrm{ba}_{3} \mathrm{a}_{1}}\left(2 \eta^{\mu_{2} \mu_{3}} \eta^{\mu_{1} \mu_{4}}-\eta^{\mu_{1} \mu_{2}} \eta^{\mu_{3} \mu_{4}}-\eta^{\mu_{1} \mu_{3}} \eta^{\mu_{2} \mu_{4}}\right)\right] .
\end{aligned}
$$

Nonetheless eq. (3.15) is equal to eq. (3.11) courtesy of the Jacobi relation $\sum_{r=1}^{3} c_{(r)}=$ 0. Eq. (3.15) simply corresponds to a different way of parceling the four-gluon vertex among the color factors $c_{(r)}$, and the expressions $n_{(r)}$ will differ by a generalized gauge transformation (2.5). The amplitude (3.1) of course remains unchanged.

\subsection{Kinematic numerators of the four-gluon amplitude}

Using the fact that the three-gluon vertex (3.5) is linear in momenta, we rewrite eq. (3.8) using

$$
V^{\nu \mu_{2} \mu_{3}}\left(k_{1}+k_{4}, k_{2}, k_{3}\right)=\left(1+k_{4 \gamma} \frac{\partial}{\partial k_{1 \gamma}}\right) V^{\nu \mu_{2} \mu_{3}}\left(k_{1}, k_{2}, k_{3}\right)
$$


and then combine eq. (3.8) and (3.13) to obtain

$$
\begin{aligned}
n_{(1)}= & \left.n_{(1)}\right|_{\text {leg }}+\left.n_{(1)}\right|_{\text {vertex }} \\
= & g^{2} \varepsilon_{1 \mu_{1}}\left[\varepsilon_{4} \cdot k_{1} \delta_{\nu}^{\mu_{1}}-i \varepsilon_{4 \alpha} k_{4 \beta}\left(S_{1}^{\alpha \beta}\right)^{\mu_{1}}-i \varepsilon_{4 \alpha} k_{4 \beta} L_{1}^{\alpha \beta} \delta_{\nu}^{\mu_{1}}\right] V^{\nu \mu_{2} \mu_{3}}\left(k_{1}, k_{2}, k_{3}\right) \varepsilon_{2 \mu_{2}} \varepsilon_{3 \mu_{3}} \\
& +g^{2} \varepsilon_{1 \mu_{1}}\left[-i \varepsilon_{4 \alpha} k_{4 \beta}\left(S_{1}^{\alpha \beta}\right)_{\nu}^{\mu_{1}}\right]\left(k_{4 \gamma} \frac{\partial}{\partial k_{1 \gamma}}\right) V^{\nu \mu_{2} \mu_{3}}\left(k_{1}, k_{2}, k_{3}\right) \varepsilon_{2 \mu_{2}} \varepsilon_{3 \mu_{3}}
\end{aligned}
$$

where the orbital angular momentum operators are defined as

$$
L_{r}^{\alpha \beta}=i\left(k_{r}^{\alpha} \frac{\partial}{\partial k_{r \beta}}-k_{r}^{\beta} \frac{\partial}{\partial k_{r \alpha}}\right) .
$$

These satisfy the Lorentz algebra commutation relations

$$
\left[L_{r}^{\alpha \beta}, L_{r}^{\gamma \delta}\right]=-i\left[\eta^{\alpha \gamma} L_{r}^{\beta \delta}-\eta^{\alpha \delta} L_{r}^{\beta \gamma}-\eta^{\beta \gamma} L_{r}^{\alpha \delta}+\eta^{\beta \delta} L_{r}^{\alpha \gamma}\right]
$$

Similar expressions are obtained for $n_{(2)}$ and $n_{(3)}$. Finally, defining the total angular momentum operator

$$
\left(J_{r}^{\alpha \beta}\right)_{\nu}^{\mu_{r}}=L_{r}^{\alpha \beta} \delta_{\nu}^{\mu_{r}}+\left(S_{r}^{\alpha \beta}\right)_{\nu}^{\mu_{r}}
$$

we can write all the kinematic numerators as

$$
n_{(r)}=g^{2}\left[\varepsilon_{4} \cdot k_{r}-i \varepsilon_{4 \alpha} k_{4 \beta} J_{r}^{\alpha \beta}-i \varepsilon_{4 \alpha} k_{4 \beta} k_{4 \gamma} S_{r}^{\alpha \beta} \frac{\partial}{\partial k_{r \gamma}}\right] V\left(k_{1}, k_{2}, k_{3}\right)
$$

where we have suppressed the polarization vectors $\varepsilon_{1}, \varepsilon_{2}$, and $\varepsilon_{3}$. The subscripts on $J_{r}$ and $S_{r}$ indicate on which polarization indices these operators act.

Note that under a gauge transformation $\varepsilon_{4} \rightarrow \varepsilon_{4}+\lambda k_{4}$ of gluon 4 , the last two terms in eq. (3.21) vanish by virtue of the antisymmetry of $J_{r}^{\alpha \beta}$ and $S_{r}^{\alpha \beta}$, and the first term gives $n_{(r)} \rightarrow n_{(r)}+\lambda g^{2} k_{4} \cdot k_{r}$ in accord with eq. (2.5).

We observe that the operators $L_{r}^{\alpha \beta}$ and $S_{r}^{\alpha \beta}$ are the same as those appearing in the Burnett-Kroll form [56] of the subleading terms of the Low soft-photon theorem [57] as applied to gluons [58-62]. (See refs. [63-65] for recent derivations of the soft-gluon theorem from gauge invariance.) The first two terms in eq. (3.21) correspond precisely to the leading and subleading terms in the $k_{4} \rightarrow 0$ expansion of the four-gluon amplitude; the third term is higher order in the soft momentum. We emphasize, however, that eq. (3.21), and the vertex expansion that we will derive in the next section, are exact, and not dependent on taking a soft limit.

\subsection{Kinematic Jacobi relation}

We will now show that the sum of the four-gluon kinematic numerators (3.21) vanishes. This result, which has been known at least since 1980 [23, 24] inspired the conjecture of color-kinematic duality [1]. We demonstrate it in a way that will facilitate the proof of color-factor symmetry of the $n$-gluon amplitude. 
Using eq. (3.21), we see that

$$
\begin{aligned}
\sum_{r=1}^{3} n_{(r)}= & g^{2}\left(\sum_{r=1}^{3} \varepsilon_{4} \cdot k_{r}\right) V\left(k_{1}, k_{2}, k_{3}\right) \\
& -i g^{2} \varepsilon_{4 \alpha} k_{4 \beta}\left(\sum_{r=1}^{3} J_{r}^{\alpha \beta}\right) V\left(k_{1}, k_{2}, k_{3}\right) \\
& -i g^{2} \varepsilon_{4 \alpha} k_{4 \beta} k_{4 \gamma} \sum_{r=1}^{3} S_{r}^{\alpha \beta} \frac{\partial}{\partial k_{r \gamma}} V\left(k_{1}, k_{2}, k_{3}\right) .
\end{aligned}
$$

The first sum on the right-hand side of eq. (3.22) vanishes by momentum conservation $\sum_{r=1}^{4} k_{r}=0$ together with $\varepsilon_{4} \cdot k_{4}=0$. The second sum on the right hand side of eq. (3.22), which may be written more explicitly as

$$
\left(J_{1}^{\alpha \beta}\right)_{\nu}^{\mu_{1}} V^{\nu \mu_{2} \mu_{3}}\left(k_{1}, k_{2}, k_{3}\right)+\left(J_{2}^{\alpha \beta}\right)_{\nu}^{\mu_{2}} V^{\mu_{1} \nu \mu_{3}}\left(k_{1}, k_{2}, k_{3}\right)+\left(J_{3}^{\alpha \beta}\right)_{\nu}^{\mu_{3}} V^{\mu_{1} \mu_{2} \nu}\left(k_{1}, k_{2}, k_{3}\right)
$$

is the first-order Lorentz transformation of the three-gluon vertex. This vanishes, as may be verified by explicit computation, because $V^{\mu_{1} \mu_{2} \mu_{3}}\left(k_{1}, k_{2}, k_{3}\right)$ is a Lorentz tensor. Alternatively, we can define the spin-one angular momentum operator to act on polarization indices $[63,64]$

$$
S_{r}^{\alpha \beta}=i\left(\varepsilon_{r}^{\alpha} \frac{\partial}{\partial \varepsilon_{r \beta}}-\varepsilon_{r}^{\beta} \frac{\partial}{\partial \varepsilon_{r \alpha}}\right)
$$

in which case

$$
\left(\sum_{r=1}^{3} J_{r}^{\alpha \beta}\right) V\left(k_{1}, k_{2}, k_{3}\right)=i \sum_{r=1}^{3}\left(k_{r}^{\alpha} \frac{\partial}{\partial k_{r \beta}}-k_{r}^{\beta} \frac{\partial}{\partial k_{r \alpha}}+\varepsilon_{r}^{\alpha} \frac{\partial}{\partial \varepsilon_{r \beta}}-\varepsilon_{r}^{\beta} \frac{\partial}{\partial \varepsilon_{r \alpha}}\right) V\left(k_{1}, k_{2}, k_{3}\right)
$$

where

$$
V\left(k_{1}, k_{2}, k_{3}\right)=\varepsilon_{1} \cdot \varepsilon_{2} \varepsilon_{3} \cdot\left(k_{2}-k_{1}\right)+\varepsilon_{2} \cdot \varepsilon_{3} \varepsilon_{1} \cdot\left(k_{3}-k_{2}\right)+\varepsilon_{3} \cdot \varepsilon_{1} \varepsilon_{2} \cdot\left(k_{1}-k_{3}\right) .
$$

Eq. (3.25) vanishes because $V\left(k_{1}, k_{2}, k_{3}\right)$ is a Lorentz-invariant function of $k_{r}$ and $\varepsilon_{r}$. The third sum in eq. (3.22) is proportional to

$$
\begin{aligned}
& \left(S_{1}^{\alpha \beta}\right)^{\mu_{1}} \frac{\partial}{\partial k_{1 \gamma}} V^{\nu \mu_{2} \mu_{3}}\left(k_{1}, k_{2}, k_{3}\right)+\left(S_{2}^{\alpha \beta}\right)^{\mu_{2}} \frac{\partial}{\partial k_{2 \gamma}} V^{\mu_{1} \nu \mu_{3}}\left(k_{1}, k_{2}, k_{3}\right)+\left(S_{3}^{\alpha \beta}\right)^{\mu_{3}}{ }_{\nu} \frac{\partial}{\partial k_{3 \gamma}} V^{\mu_{1} \mu_{2} \nu}\left(k_{1}, k_{2}, k_{3}\right) \\
& =2 i\left(-\eta^{\alpha \mu_{1}} \eta^{\beta \mu_{2}} \eta^{\gamma \mu_{3}}+\eta^{\alpha \mu_{1}} \eta^{\gamma \mu_{2}} \eta^{\beta \mu_{3}}\right)+(\text { cyclic permutations of 123). }
\end{aligned}
$$

This expression is antisymmetric in $\beta$ and $\gamma$. Since it multiplies $k_{4 \beta} k_{4 \gamma}$ in eq. (3.22), the whole expression vanishes. The cancellations that we have just exhibited were originally used in ref. [35] to prove the radiation zero theorem.

We have shown that the sum of kinematic numerators vanishes (the kinematic Jacobi relation), and thus have demonstrated the invariance of the four-gluon amplitude $\delta_{4} \mathcal{A}_{4}=0$ under the color-factor shift symmetry. We would like to emphasize that in proving the vanishing of eq. (3.23) and (3.27) we did not use that $k_{r}$ were on-shell, nor did we use $\varepsilon_{r} \cdot k_{r}=0$ for $r=1,2,3$. Thus we will be able to use these results in section 4 for an off-shell three-gluon vertex $V^{\mu_{1} \mu_{2} \mu_{3}}\left(k_{1}, k_{2}, k_{3}\right)$. 


\section{Proof of color-factor symmetry for $n$-gluon amplitudes}

We now turn to the proof that the tree-level $n$-gluon amplitude is invariant under a colorfactor shift associated with any of the gluons. To do so, we employ a decomposition of the amplitude known as the radiation vertex expansion [35]. This is a recursive approach which constructs an $n$-point amplitude by attaching a massless vector boson to all possible $(n-1)$-point diagrams.

Consider the set of all tree-level $(n-1)$-gluon diagrams with external legs $\{1, \cdots, n\} \backslash$ $\{a\}$ constructed using Feynman rules. Label these diagrams by $I^{\prime}$. Please note that this set differs from the set of $(n-1)$-point diagrams appearing in the cubic vertex expansion (cf. section 2) because it includes not only cubic diagrams but also diagrams with four-gluon vertices. (In the cubic decomposition (2.8), all diagrams containing quartic vertices are redistributed among the cubic diagrams.)

We will construct all possible $n$-gluon diagrams by attaching gluon $a$ to each $(n-1)$ gluon diagram $I^{\prime}$ in all possible ways. This includes: (1) attaching gluon $a$ to an external leg, (2) attaching gluon $a$ to an internal line, or (3) attaching gluon $a$ to one of the threegluon vertices of $I^{\prime}$ to make a four-gluon vertex. By rearranging terms and discarding pieces that vanish by Ward identities, we obtain simple expressions for the contribution to the $n$-gluon amplitude from each vertex of diagram $I^{\prime}$. We then show that each such contribution is invariant under the color-factor shift associated with gluon $a$.

\subsection{Attaching a gluon to an external leg}

First we single out one of the external legs, $b$, of $I^{\prime}$, denoting the resulting expression as

$$
\varepsilon_{b \mu_{b}} B_{\mathrm{a}_{b}}^{\mu_{b}}\left(k_{b}, \cdots\right)
$$

where $k_{b}$ and $\varepsilon_{b}$ are the momentum and polarization vector of gluon $b$, and $\cdots$ denotes momenta belonging to gluons $\{1, \cdots, n\} \backslash\{a, b\}$. Attaching gluon $a$ to external leg $b$ and using eq. (3.5) we obtain

$$
\begin{aligned}
&-\frac{g}{\sqrt{2}} \frac{f_{\mathrm{a}_{b} \mathrm{a}_{\mathrm{b}} \mathrm{b}}}{\left(k_{a}+k_{b}\right)^{2}} V^{\mu_{b} \mu_{a} \nu}\left(k_{b}, k_{a},-k_{a}-k_{b}\right) B_{\mathrm{b} \nu}\left(k_{a}+k_{b}, \cdots\right) \\
&=-\frac{g}{\sqrt{2}} \frac{f_{\mathrm{a}_{b} \mathrm{a}_{a} \mathrm{~b}}}{\left(k_{a}+k_{b}\right)^{2}}\left[\eta^{\mu_{b} \nu} k_{a}^{\mu_{a}}-\eta^{\mu_{a} \nu} k_{b}^{\mu_{b}}-\eta^{\mu_{b} \mu_{a}}\left(k_{a}+k_{b}\right)^{\nu}\right. \\
&\left.\quad+2 \eta^{\mu_{b} \nu} k_{b}^{\mu_{a}}+2\left(\eta^{\mu_{b} \mu_{a}} k_{a}^{\nu}-\eta^{\mu_{a} \nu} k_{a}^{\mu_{b}}\right)\right] B_{\mathrm{b} \nu}\left(k_{a}+k_{b}, \cdots\right) .
\end{aligned}
$$

Contracting this with $\varepsilon_{b \mu_{b}} \varepsilon_{a \mu_{a}}$ eliminates the first two terms in the square brackets. The third term is proportional to

$$
\left(k_{a}+k_{b}\right)^{\nu} B_{\mathrm{b} \nu}\left(k_{a}+k_{b}, \cdots\right) .
$$

This term does not vanish by itself as it did in the case of the four-gluon amplitude, but when we obtain the total $n$-gluon amplitude by including all $(n-1)$-point diagrams $I^{\prime}$, the sum of such terms vanishes due to gauge invariance (Ward identity). Therefore we are 
left with the last two terms in square brackets, which yield the two terms in the following expression

$$
-\sqrt{2} g \frac{f_{\mathrm{a}_{\mathrm{a} a} \mathrm{~b}}}{\left(k_{a}+k_{b}\right)^{2}} \varepsilon_{b \mu_{b}}\left[\varepsilon_{a} \cdot k_{b} \eta^{\mu_{b} \nu}+\left(\varepsilon_{a}^{\mu_{b}} k_{a}^{\nu}-\varepsilon_{a}^{\nu} k_{a}^{\mu_{b}}\right)\right] B_{\mathrm{b} \nu}\left(k_{a}+k_{b}, \cdots\right) .
$$

We set this expression aside for now.

\subsection{Attaching a gluon to an internal line}

Next we single out one of the internal lines of $I^{\prime}$, which divides the diagram into two subdiagrams $B$ and $C$, and splits the external legs $\{1, \cdots, n\} \backslash\{a\}$ into two complementary sets $S_{a, B}$ and $S_{a, C}$. The contribution of the diagram can thus be written as

$$
B_{\mathrm{b}}^{\mu}(-K, \cdots) \frac{\left(-i \eta_{\mu \nu}\right) \delta_{\mathrm{bc}}}{K^{2}} C_{\mathrm{c}}^{\nu}(K, \cdots)
$$

where $K=\sum_{d \in S_{a, B}} k_{d}$ is the momentum running through the line, and the $\cdots$ in $B$ and $C$ denote momenta belonging to $S_{a, B}$ and $S_{a, C}$ respectively. Attaching gluon $a$ to the line connecting the two subgraphs yields

$$
\frac{i g}{\sqrt{2}} B_{\mathrm{b} \mu}(-K, \cdots) \frac{f_{\mathrm{bca}_{a}} V^{\mu \nu \mu_{a}}\left(K,-K-k_{a}, k_{a}\right)}{K^{2}\left(K+k_{a}\right)^{2}} C_{\mathrm{c} \nu}\left(K+k_{a}, \cdots\right) .
$$

Writing the three-gluon vertex (3.5) as

$V^{\mu \nu \mu_{a}}\left(K,-K-k_{a}, k_{a}\right)=-\eta^{\mu \nu} k_{a}^{\mu_{a}}+\eta^{\mu_{a} \nu} K^{\mu}+\eta^{\mu \mu_{a}}\left(K+k_{a}\right)^{\nu}-2 \eta^{\mu \nu} K^{\mu_{a}}-2\left(\eta^{\mu \mu_{a}} k_{a}^{\nu}-\eta^{\mu_{a} \nu} k_{a}^{\mu}\right)$

we see that the first term vanishes when contracted with $\varepsilon_{a \mu_{a}}$. The second and third terms give terms proportional to

$$
K^{\mu} B_{\mathrm{b} \mu}(-K, \cdots), \quad\left(K+k_{a}\right)^{\nu} C_{\mathrm{c} \nu}\left(K+k_{a}, \cdots\right) .
$$

Again these terms do not vanish by themselves, but when we include all $(n-1)$-gluon diagrams $I^{\prime}, B_{\mathrm{b} \mu}$ will be replaced by the sum over all diagrams containing external legs $S_{a, B}$ plus one additional off-shell line, and similarly for $C_{\mathrm{c} \nu}$, and these expressions will vanish by gauge invariance (Ward identity). We are thus left with

$$
-\sqrt{2} i g B_{\mathrm{b} \mu}(-K, \cdots) \frac{f_{\mathrm{bca} a}\left[\eta^{\mu \nu} \varepsilon_{a} \cdot K+\left(\varepsilon_{a}^{\mu} k_{a}^{\nu}-\varepsilon_{a}^{\nu} k_{a}^{\mu}\right)\right]}{K^{2}\left(K+k_{a}\right)^{2}} C_{\mathrm{c} \nu}\left(K+k_{a}, \cdots\right) .
$$

Now we use the identity (2.20) to rewrite this as

$$
\begin{aligned}
& B_{\mathrm{b} \mu}(-K, \cdots) \frac{-i}{K^{2}}\left\{\sqrt{2} g \frac{f_{\mathrm{bca}_{a}}}{2 k_{a} \cdot K}\left[\eta^{\mu \nu} \varepsilon_{a} \cdot K+\left(\varepsilon_{a}^{\mu} k_{a}^{\nu}-\varepsilon_{a}^{\nu} k_{a}^{\mu}\right)\right] C_{\mathrm{c} \nu}\left(K+k_{a}, \cdots\right)\right\} \\
& +\left\{-\sqrt{2} g \frac{f_{\mathrm{bca}_{a}}}{2 k_{a} \cdot K} B_{\mathrm{b} \mu}(\cdots,-K)\left[\eta^{\mu \nu} \varepsilon_{a} \cdot K+\left(\varepsilon_{a}^{\mu} k_{a}^{\nu}-\varepsilon_{a}^{\nu} k_{a}^{\mu}\right)\right]\right\} \frac{-i}{\left(K+k_{a}\right)^{2}} C_{\mathrm{c} \nu}\left(K+k_{a}, \cdots\right) .
\end{aligned}
$$

We associate each of the terms in this equation with one of the two vertices to which the line is attached. 


\subsection{Radiation vertex expansion}

In the previous subsections, we showed that attaching gluon $a$ to an $(n-1)$-gluon diagram yields one term (4.4) for each external leg and two terms (4.10) for each internal line, or in other words, one term for each leg of each vertex of $I^{\prime}$. We can therefore reorganize these terms into a sum over the legs of the vertices of each of the $(n-1)$-gluon diagrams $I^{\prime}$. This is the radiation vertex expansion [35].

First we choose one of the three-gluon vertices $v$ of $I^{\prime}$ (if it has any). Such a vertex divides the external legs into three non-overlapping subsets $S_{\left(a, I^{\prime}, v, r\right)}, r=1,2,3$ such that $\bigcup_{r=1}^{3} S_{\left(a, I^{\prime}, v, r\right)}=\{1, \cdots, n\} \backslash\{a\}$. The contribution of diagram $I^{\prime}$ to the $(n-1)$-gluon amplitude can be expressed

$$
-\frac{i g}{\sqrt{2}} f_{\mathrm{c}_{1} \mathrm{c}_{2} \mathrm{c}_{3}} V^{\mu_{1} \mu_{2} \mu_{3}}\left(K_{1}, K_{2}, K_{3}\right) \prod_{r=1}^{3} A_{\mathrm{c}_{r} \mu_{r}}^{(r)}\left(-K_{r}, \cdots\right)
$$

where $K_{r}=\sum_{d \in S_{\left(a, I^{\prime}, v, r\right)}} k_{d}$ is the momentum flowing out of each leg of the vertex, and $\cdots$ in $A^{(r)}$ denotes momenta belonging to $S_{\left(a, I^{\prime}, v, r\right)}$. If any of the legs is external, then $A_{c_{r} \mu_{r}}^{(r)}$ is just $\delta_{\mathrm{bc}_{r}} \varepsilon_{b \mu_{b}}$.

We now attach gluon $a$ to each of the legs of this three-gluon vertex, either to an external leg or to an internal line. From eq. (4.4) and (4.10) this yields

$$
\begin{aligned}
i g^{2} \prod_{r=1}^{3} A_{c_{r} \mu_{r}}^{(r)}\left(-K_{r}, \cdots\right) & \left(\frac{f_{\mathrm{bc}_{1} \mathrm{a}_{a}} f_{\mathrm{bc}_{2} \mathrm{c}_{3}}}{2 k_{a} \cdot K_{1}}\left[\eta^{\mu_{1} \nu} \varepsilon_{a} \cdot K_{1}+\left(\varepsilon_{a}^{\mu_{1}} k_{a}^{\nu}-\varepsilon_{a}^{\nu} k_{a}^{\mu_{1}}\right)\right] V_{\nu}{ }_{2}^{\mu_{2} \mu_{3}}\left(K_{1}+k_{a}, K_{2}, K_{3}\right)\right. \\
& +\frac{f_{\mathrm{bc}_{2} \mathrm{a}_{a}} f_{\mathrm{bc}_{3} \mathrm{c}_{1}}}{2 k_{a} \cdot K_{2}}\left[\eta^{\mu_{2} \nu} \varepsilon_{a} \cdot K_{2}+\left(\varepsilon_{a}^{\mu_{2}} k_{a}^{\nu}-\varepsilon_{a}^{\nu} k_{a}^{\mu_{2}}\right)\right] V_{\nu}^{\mu_{1}}{ }_{\nu}^{\mu_{3}}\left(K_{1}, K_{2}+k_{a}, K_{3}\right) \\
& \left.+\frac{f_{\mathrm{bc}_{3} \mathrm{a}_{a}} f_{\mathrm{bc}_{1} \mathrm{c}_{2}}}{2 k_{a} \cdot K_{3}}\left[\eta^{\mu_{3} \nu} \varepsilon_{a} \cdot K_{3}+\left(\varepsilon_{a}^{\mu_{3}} k_{a}^{\nu}-\varepsilon_{a}^{\nu} k_{a}^{\mu_{3}}\right)\right] V_{\nu}^{\mu_{1} \mu_{2}}\left(K_{1}, K_{2}, K_{3}+k_{a}\right)\right) .
\end{aligned}
$$

We can also attach gluon $a$ directly to the three-gluon vertex itself. Using eq. (3.12), this yields

$$
\begin{aligned}
-\frac{i g^{2}}{2} \prod_{r=1}^{3} A_{\mathrm{c}_{r} \mu_{r}}^{(r)}\left(-K_{r}, \cdots\right)( & f_{\mathrm{bc}_{1} \mathrm{a}_{a}} f_{\mathrm{bc}_{2} \mathrm{c}_{3}} \varepsilon_{a \mu_{a}} \frac{\partial}{\partial K_{1 \mu_{a}}} V^{\mu_{1} \mu_{2} \mu_{3}}\left(K_{1}, K_{2}, K_{3}\right) \\
& +f_{\mathrm{bc}_{2} \mathrm{a}_{a}} f_{\mathrm{bc}_{3} \mathrm{c}_{1}} \varepsilon_{a \mu_{a}} \frac{\partial}{\partial K_{2 \mu_{a}}} V^{\mu_{1} \mu_{2} \mu_{3}}\left(K_{1}, K_{2}, K_{3}\right) \\
& \left.+f_{\mathrm{bc}_{3} \mathrm{a}_{a} f_{\mathrm{bc}_{1} \mathrm{c}_{2}}} \varepsilon_{a \mu_{a}} \frac{\partial}{\partial K_{3 \mu_{a}}} V^{\mu_{1} \mu_{2} \mu_{3}}\left(K_{1}, K_{2}, K_{3}\right)\right) .
\end{aligned}
$$

We now use eq. (3.16) in eq. (4.12), and combine eq. (4.12) and (4.13) as we did in section 3. Leaving the indices on $V^{\mu_{1} \mu_{2} \mu_{3}}\left(K_{1}, K_{2}, K_{3}\right)$ implicit, we obtain the contribution of the three-gluon vertex to the radiation vertex expansion

$$
\begin{aligned}
i g^{2} \prod_{r=1}^{3} A_{c_{r}}^{(r)}\left(-K_{r}, \cdots\right) & \left(\frac{f_{\mathrm{bc}_{1} \mathrm{a}_{a}} f_{\mathrm{bc}_{2} \mathrm{c}_{3}}}{2 k_{a} \cdot K_{1}}\left[\varepsilon_{a} \cdot K_{1}-i \varepsilon_{a \alpha} k_{a \beta} J_{1}^{\alpha \beta}-i \varepsilon_{a \alpha} k_{a \beta} k_{a \gamma} S_{1}^{\alpha \beta} \frac{\partial}{\partial K_{1 \gamma}}\right] V\left(K_{1}, K_{2}, K_{3}\right)\right. \\
& +\frac{f_{\mathrm{bc}_{2} \mathrm{a}_{a}} f_{\mathrm{bc}_{3} \mathrm{c}_{1}}}{2 k_{a} \cdot K_{2}}\left[\varepsilon_{a} \cdot K_{2}-i \varepsilon_{a \alpha} k_{a \beta} J_{2}^{\alpha \beta}-i \varepsilon_{a \alpha} k_{a \beta} k_{a \gamma} S_{2}^{\alpha \beta} \frac{\partial}{\partial K_{2 \gamma}}\right] V\left(K_{1}, K_{2}, K_{3}\right) \\
& \left.+\frac{f_{\mathrm{bc}_{3} \mathrm{a}_{a}} f_{\mathrm{bc}_{1} \mathrm{c}_{2}}}{2 k_{a} \cdot K_{3}}\left[\varepsilon_{a} \cdot K_{3}-i \varepsilon_{a \alpha} k_{a \beta} J_{3}^{\alpha \beta}-i \varepsilon_{a \alpha} k_{a \beta} k_{a \gamma} S_{3}^{\alpha \beta} \frac{\partial}{\partial K_{3 \gamma}}\right] V\left(K_{1}, K_{2}, K_{3}\right)\right)
\end{aligned}
$$


where the subscripts on $J_{r}$ and $S_{r}$ indicate on which indices of $V^{\mu_{1} \mu_{2} \mu_{3}}\left(K_{1}, K_{2}, K_{3}\right)$ these operators act.

Next we choose one of the four-gluon vertices $v$ of $I^{\prime}$ (if it has any). Such a vertex divides the external legs into four non-overlapping subsets $S_{\left(a, I^{\prime}, v, r\right)}, r=1, \cdots, 4$ such that $\bigcup_{r=1}^{4} S_{\left(a, I^{\prime}, v, r\right)}=\{1, \cdots, n\} \backslash\{a\}$. The contribution of the diagram $I^{\prime}$ can be expressed as

$$
V_{\mathrm{c}_{1} \mathrm{c}_{2} \mathrm{c}_{3} \mathrm{c}_{4}}^{\mu_{1} \mu_{2} \mu_{3}} \prod_{r=1}^{4} A_{\mathrm{c}_{r} \mu_{r}}^{(r)}\left(-K_{r}, \cdots\right) .
$$

We now attach gluon $a$ to each of the legs of this four-gluon vertex. From eq. (4.4) and (4.10) we obtain

$$
\begin{aligned}
-\sqrt{2} g \prod_{r=1}^{4} A_{\mathrm{c}_{r} \mu_{r}}^{(r)}\left(-K_{r}, \cdots\right)( & \frac{f_{\mathrm{bc}_{1} \mathrm{a}_{a}}}{2 k_{a} \cdot K_{1}}\left[\eta^{\mu_{1} \nu} \varepsilon_{a} \cdot K_{1}+\left(\varepsilon_{a}^{\mu_{1}} k_{a}^{\nu}-\varepsilon_{a}^{\nu} k_{a}^{\mu_{1}}\right)\right] \eta_{\nu \lambda} V_{\mathrm{bc}_{2} \mathrm{c}_{3} \mathrm{c}_{4}}^{\lambda \mu_{2} \mu_{3} \mu_{4}} \\
& +\frac{f_{\mathrm{bc}_{2} \mathrm{a}_{a}}}{2 k_{a} \cdot K_{2}}\left[\eta^{\mu_{2} \nu} \varepsilon_{a} \cdot K_{2}+\left(\varepsilon_{a}^{\mu_{2}} k_{a}^{\nu}-\varepsilon_{a}^{\nu} k_{a}^{\mu_{2}}\right)\right] \eta_{\nu \lambda} V_{\mathrm{c}_{1} \mathrm{bc}_{3} \mathrm{c}_{4}}^{\mu_{1} \lambda \mu_{3} \mu_{4}} \\
& +\frac{f_{\mathrm{bc}_{3} \mathrm{a}_{a}}}{2 k_{a} \cdot K_{3}}\left[\eta^{\mu_{3} \nu} \varepsilon_{a} \cdot K_{3}+\left(\varepsilon_{a}^{\mu_{3}} k_{a}^{\nu}-\varepsilon_{a}^{\nu} k_{a}^{\mu_{3}}\right)\right] \eta_{\nu \lambda} V_{\mathrm{c}_{1} \mathrm{c}_{2} \mathrm{~b}_{4}}^{\mu_{1} \mu_{2} \lambda \mu_{4}} \\
& \left.+\frac{f_{\mathrm{bc}_{4} \mathrm{a}_{a}}}{2 k_{a} \cdot K_{4}}\left[\eta^{\mu_{4} \nu} \varepsilon_{a} \cdot K_{4}+\left(\varepsilon_{a}^{\mu_{4}} k_{a}^{\nu}-\varepsilon_{a}^{\nu} k_{a}^{\mu_{4}}\right)\right] \eta_{\nu \lambda} V_{\mathrm{c}_{1} \mathrm{c}_{2} \mathrm{c}_{3} \mathrm{~b}}^{\mu_{1} \mu_{2} \mu_{3} \lambda}\right) .
\end{aligned}
$$

One cannot attach gluon $a$ to the four-gluon vertex itself since there are no five-gluon vertices. Thus the contribution to the radiation vertex expansion from the four-gluon vertex is

$$
\begin{aligned}
-\sqrt{2} g \prod_{r=1}^{4} A_{\mathrm{c}_{r} \mu_{r}}^{(r)}\left(-K_{r}, \cdots\right)( & \frac{f_{\mathrm{bc}_{1} \mathrm{a}_{a}}}{2 k_{a} \cdot K_{1}}\left[\delta_{\nu}^{\mu_{1}} \varepsilon_{a} \cdot K_{1}-i \varepsilon_{a \alpha} k_{a \beta}\left(S_{1}^{\alpha \beta}\right)^{\mu_{1}}\right] V_{\mathrm{bc}_{2} \mathrm{c}_{3} \mathrm{c}_{4}}^{\nu \mu_{2} \mu_{3} \mu_{4}} \\
& +\frac{f_{\mathrm{bc}_{2} \mathrm{a}_{a}}}{2 k_{a} \cdot K_{2}}\left[\delta^{\mu_{2}} \varepsilon_{a} \cdot K_{2}-i \varepsilon_{a \alpha} k_{a \beta}\left(S_{2}^{\alpha \beta}\right)^{\mu_{2}}{ }_{\nu}\right] V_{\mathrm{c}_{1} \mathrm{~b}_{3} \mathrm{c}_{4}}^{\mu_{1} \mu_{\mu_{4}}} \\
& +\frac{f_{\mathrm{bc}_{3} \mathrm{a}_{a}}}{2 k_{a} \cdot K_{3}}\left[\delta_{\nu}^{\mu_{3}} \varepsilon_{a} \cdot K_{3}-i \varepsilon_{a \alpha} k_{a \beta}\left(S_{3}^{\alpha \beta}\right)^{\mu_{3}}{ }_{\nu}\right] V_{\mathrm{c}_{1} \mathrm{c}_{2} \mathrm{bc}_{4}}^{\mu_{1} \mu_{2} \nu \mu_{4}} \\
& \left.+\frac{f_{\mathrm{bc}_{4} \mathrm{a}_{a}}}{2 k_{a} \cdot K_{4}}\left[\delta_{\nu}^{\mu_{4}} \varepsilon_{a} \cdot K_{4}-i \varepsilon_{a \alpha} k_{a \beta}\left(S_{4}^{\alpha \beta}\right)^{\mu_{4}}\right] V_{\mathrm{c}_{1} \mathrm{c}_{2} \mathrm{c}_{3} \mathrm{~b}}^{\mu_{1} \mu_{3} \mu_{3}}\right) .
\end{aligned}
$$

To summarize this section, we have expressed an $n$-gluon amplitude as a sum over the vertices of all of the $(n-1)$-gluon diagrams $I^{\prime}$, comprising a term (4.14) for each three-gluon vertex of $I^{\prime}$ and a term (4.17) for each four-gluon vertex of $I^{\prime}$.

\subsection{Invariance of the radiation vertex expansion under color-factor symmetry}

Computing the variation of the radiation vertex expansion of the amplitude under a colorfactor shift is somewhat more delicate than calculating the variation of the cubic vertex expansion of the amplitude (as we did in section 2) because each factor $A_{c_{r} \mu_{r}}^{(r)}$ in eq. (4.14) and (4.17) can contain more than one color factor $c_{i}$ due to the possible presence of fourgluon vertices.

First let us consider the contribution (4.14) of a three-gluon vertex to the radiation vertex expansion, and for the moment let us assume that the subdiagrams corresponding 
to $A_{c_{r} \mu_{r}}^{(r)}$ contain only three-gluon vertices. Designate by $c_{\left(a, I^{\prime}, v, r\right)}$ with $r=1,2,3$ the color factor associated with each line of eq. (4.14). Thus, for example, $c_{\left(a, I^{\prime}, v, 1\right)}$ is the product of $f_{\mathrm{bc}_{1} \mathrm{a}_{a}} f_{\mathrm{bc}_{2} \mathrm{c}_{3}}$ and the structure constants from all the three-gluon vertices in $\prod_{r=1}^{3} A_{\mathrm{c}_{r} \mu_{r}}^{(r)}$. These color factors manifestly satisfy $\sum_{r=1}^{3} c_{\left(a, I^{\prime}, v, r\right)}=0$. The variation of $c_{\left(a, I^{\prime}, v, r\right)}$ under the color-factor shift associated with gluon $a$ is

$$
\delta_{a} c_{\left(a, I^{\prime}, v, r\right)}=\alpha_{\left(a, I^{\prime}, v\right)} k_{a} \cdot K_{r}
$$

which preserves $\sum_{r=1}^{3} c_{\left(a, I^{\prime}, v, r\right)}=0$. The variation of eq. (4.14) under the color-factor shift is therefore proportional to

$$
\left[\left(\sum_{r=1}^{3} \varepsilon_{a} \cdot K_{r}\right)-i \varepsilon_{a \alpha} k_{a \beta}\left(\sum_{r=1}^{3} J_{r}^{\alpha \beta}\right)-i \varepsilon_{a \alpha} k_{a \beta} k_{a \gamma}\left(\sum_{r=1}^{3} S_{r}^{\alpha \beta} \frac{\partial}{\partial K_{r \gamma}}\right)\right] V\left(K_{1}, K_{2}, K_{3}\right) .
$$

In section 3, we demonstrated that each of the three terms in eq. (4.19) vanishes. If the diagrams corresponding to $A_{\mathcal{c}_{r} \mu_{r}}^{(r)}$ contain four-gluon vertices, we can use eq. (3.11) to expand these expressions and then use the argument above to show that each separate contribution will vanish under the color-factor shift. Therefore the contribution of the three-gluon vertices (4.14) to the radiation vertex expansion is invariant under the colorfactor shift associated with gluon $a$.

Second let us consider the contribution (4.17) of a four-gluon vertex to the radiation vertex expansion. Again we begin by assuming that the diagrams corresponding to $A_{c_{r} \mu_{r}}^{(r)}$ contain only three-gluon vertices. We now expand the four-gluon vertices $V_{\mathrm{C}_{1} \mathrm{C}_{2} \mathrm{C}_{3} \mathrm{C}_{4}}^{\mu_{1} \mu_{2} \mu_{3} \mu_{4}}$ in eq. (4.17) into several terms, one of which is

$$
\begin{aligned}
& -\sqrt{2} g \prod_{r=1}^{4} A_{\mathrm{c}_{r} \mu_{r}}^{(r)}\left(-K_{r}, \cdots\right) \\
& \times\left(\frac{f_{\mathrm{bc}_{1} \mathrm{a}_{a}} f_{\mathrm{bc}_{2} \mathrm{~d}} f_{\mathrm{dc}_{3} \mathrm{c}_{4}}}{2 k_{a} \cdot K_{1}}\left[\delta_{\nu}^{\mu_{1}} \varepsilon_{a} \cdot K_{1}-i \varepsilon_{a \alpha} k_{a \beta}\left(S_{1}^{\alpha \beta}\right)^{\mu_{1}}\right]\left(\eta^{\nu \mu_{3}} \eta^{\mu_{2} \mu_{4}}-\eta^{\nu \mu_{4}} \eta^{\mu_{2} \mu_{3}}\right)\right. \\
& \quad+\frac{f_{\mathrm{bc}_{2} \mathrm{a}_{a}} f_{\mathrm{c}_{\mathrm{b}} \mathrm{bd}} f_{\mathrm{dc}_{3} \mathrm{c}_{4}}}{2 k_{a} \cdot K_{2}}\left[\delta_{\nu}^{\mu_{2}} \varepsilon_{a} \cdot K_{2}-i \varepsilon_{a \alpha} k_{a \beta}\left(S_{2}^{\alpha \beta}\right)^{\mu_{2}}{ }_{\nu}\right]\left(\eta^{\mu_{1} \mu_{3}} \eta^{\nu \mu_{4}}-\eta^{\mu_{1} \mu_{4}} \eta^{\nu \mu_{3}}\right) \\
& \quad+\frac{f_{\mathrm{bc}_{3} \mathrm{a}_{a}} f_{b \mathrm{c}_{4} \mathrm{~d}} f_{\mathrm{dc}_{1} \mathrm{c}_{2}}}{2 k_{a} \cdot K_{3}}\left[\delta_{\nu}^{\mu_{3}} \varepsilon_{a} \cdot K_{3}-i \varepsilon_{a \alpha} k_{a \beta}\left(S_{3}^{\alpha \beta}\right)_{\nu}^{\mu_{3}}\right]\left(\eta^{\mu_{1} \nu} \eta^{\mu_{2} \mu_{4}}-\eta^{\mu_{1} \mu_{4}} \eta^{\mu_{2} \nu}\right) \\
& \quad+\frac{\left.f_{\mathrm{bc}_{4} \mathrm{a}_{a}{ }_{\mathrm{c}_{3} \mathrm{bd}} f_{\mathrm{dc}_{1} \mathrm{c}_{2}}}^{2 k_{a} \cdot K_{4}}\left[\delta^{\mu_{4}} \varepsilon_{a} \cdot K_{4}-i \varepsilon_{a \alpha} k_{a \beta}\left(S_{4}^{\alpha \beta}\right)_{\nu}^{\mu_{4}}\right]\left(\eta^{\mu_{1} \mu_{3}} \eta^{\mu_{2} \nu}-\eta^{\mu_{1} \nu} \eta^{\mu_{2} \mu_{3}}\right)\right) .}{}
\end{aligned}
$$

Designate by $c_{\left(a, I^{\prime}, v, r\right)}$ with $r=1, \cdots 4$ the color factor associated with each line of eq. (4.20), including the structure constants from all the three-gluon vertices in $\prod_{r=1}^{4} A_{c_{r} \mu_{r}}^{(r)}$. These color factors satisfy $\sum_{r=1}^{4} c_{\left(a, I^{\prime}, v, r\right)}=0$ by virtue of

$$
f_{\mathrm{bc}_{1} \mathrm{a}_{a}} f_{b c_{2} \mathrm{~d}} f_{\mathrm{dc}_{3} \mathrm{c}_{4}}+f_{\mathrm{bc}_{2} \mathrm{a}_{a}} f_{\mathrm{c}_{1} \mathrm{bd}} f_{\mathrm{dc}_{3} \mathrm{c}_{4}}+f_{\mathrm{bc}_{3} \mathrm{a}_{a}} f_{b_{c_{4} \mathrm{~d}}} f_{\mathrm{dc}_{1} \mathrm{c}_{2}}+f_{\mathrm{bc}_{4} \mathrm{a}_{a}} f_{\mathrm{c}_{3} \mathrm{bd}} f_{\mathrm{dc}_{1} \mathrm{c}_{2}}=0 .
$$

The variation of $c_{\left(a, I^{\prime}, v, r\right)}$ under the color-factor shift associated with gluon $a$ is

$$
\delta_{a} c_{\left(a, I^{\prime}, v, r\right)}=\alpha_{\left(a, I^{\prime}, v\right)} k_{a} \cdot K_{r} .
$$


The variation of eq. (4.20) under the color-factor shift therefore contains two sums. The first

$$
\left(\sum_{r=1}^{4} \varepsilon_{a} \cdot K_{r}\right)\left(\eta^{\mu_{1} \mu_{3}} \eta^{\mu_{2} \mu_{4}}-\eta^{\mu_{1} \mu_{4}} \eta^{\mu_{2} \mu_{3}}\right)
$$

vanishes by momentum conservation, $k_{a}+\sum_{r=1}^{4} K_{r}=0$, together with $\varepsilon_{a} \cdot k_{a}=0$. The second

$$
\begin{aligned}
& \left(S_{1}^{\alpha \beta}\right)_{\nu}^{\mu_{1}}\left(\eta^{\nu \mu_{3}} \eta^{\mu_{2} \mu_{4}}-\eta^{\nu \mu_{4}} \eta^{\mu_{2} \mu_{3}}\right)+\left(S_{2}^{\alpha \beta}\right)_{\nu}^{\mu_{2}}\left(\eta^{\mu_{1} \mu_{3}} \eta^{\nu \mu_{4}}-\eta^{\mu_{1} \mu_{4}} \eta^{\nu \mu_{3}}\right) \\
& +\left(S_{3}^{\alpha \beta}\right)_{\nu}^{\mu_{3}}\left(\eta^{\mu_{1} \nu} \eta^{\mu_{2} \mu_{4}}-\eta^{\mu_{1} \mu_{4}} \eta^{\mu_{2} \nu}\right)+\left(S_{4}^{\alpha \beta}\right)^{\mu_{4}}\left(\eta^{\mu_{1} \mu_{3}} \eta^{\mu_{2} \nu}-\eta^{\mu_{1} \nu} \eta^{\mu_{2} \mu_{3}}\right)
\end{aligned}
$$

is the first-order Lorentz transformation of the tensor $\eta^{\mu_{1} \mu_{3}} \eta^{\mu_{2} \mu_{4}}-\eta^{\mu_{1} \mu_{4}} \eta^{\mu_{2} \mu_{3}}$, which vanishes. The variation under the color-factor shift of the other two terms from the expansion of $V_{\mathrm{C}_{1} \mathrm{C}_{2} \mathrm{C}_{3} \mathrm{C}_{4}}^{\mu_{1} \mu_{2} \mu_{4}}$ similarly vanishes. Furthermore, the same argument applies when the diagrams corresponding to $A_{c_{r} \mu_{r}}^{(r)}$ contain four-gluon vertices, by expanding these expressions using eq. (3.11). Therefore the contribution of the four-gluon vertices (4.17) to the radiation vertex expansion is invariant under the color-factor shift associated with gluon $a$.

In fine, we have shown that each contribution to the radiation vertex expansion is invariant under the color-factor shift associated with gluon $a$, and therefore the entire $n$-gluon amplitude is invariant under this shift. QED

\section{Color-factor symmetry for more general amplitudes}

In sections 3 and 4 , we proved the color-factor symmetry of $n$-gluon amplitudes, from which follow the BCJ relations for color-ordered amplitudes. Color-factor symmetry is a property of a much larger class of tree-level gauge-theory amplitudes, namely those containing at least one gluon together with massless or massive particles in arbitrary representations of the gauge group with arbitrary spin $\leq 1$, with the usual gauge-theory couplings. We will establish the invariance of this larger class of gauge-theory amplitudes under a color-factor shift in sections 6 and 7.

Consider a tree-level $n$-point gauge-theory amplitude $\mathcal{A}_{n}$ with gluons as well as particles $\psi$ and $\bar{\psi}$, either massless or massive, with spin zero, one-half, or one, in an arbitrary representation of the gauge group. For convenience, throughout the next three sections we refer to $\psi$ (and $\bar{\psi}$ ) as fundamentals (and antifundamentals), but they can be in any representation. This amplitude has the cubic decomposition

$$
\mathcal{A}_{n}=\sum_{i} \frac{c_{i}^{\prime} n_{i}^{\prime}}{d_{i}^{\prime}}
$$

where we decorate the color factors, kinematic numerators, and denominators with primes to distinguish them from the analogous quantities for $n$-gluon amplitudes. The denominator $d_{i}^{\prime}$ now consists of the product of inverse propagators for both massless and massive particles. The color factor $c_{i}^{\prime}$ associated with each cubic diagram is obtained by sewing together $g g g$ vertices $f_{\mathrm{abc}}$ and $\bar{\psi} g \psi$ vertices $\left(T^{\mathrm{a}}\right)^{\mathrm{i}}{ }_{\mathrm{j}}$ where $T^{\mathrm{a}}$ denote the generators in the appropriate representation. Contributions from Feynman diagrams with quartic vertices (either gggg 
or $\bar{\psi} g g \psi$ in the case of a scalar or vector $\psi$ ) are parceled out among the cubic diagrams. The number of cubic diagrams in the sum (5.1) will generally be fewer than for the $n$-gluon amplitude, as some will be excluded for violating fermion number or flavor symmetry.

Just as in the case of the $n$-gluon amplitude, the amplitude $\mathcal{A}_{n}$ can be written in a cubic vertex expansion with respect to gluon $a$ :

$$
\mathcal{A}_{n}=\sum_{I} \sum_{v} \frac{1}{\prod_{s=1}^{3} d_{(a, I, v, s)}^{\prime}} \sum_{r=1}^{3} \frac{c_{(a, I, v, r)}^{\prime} n_{(a, I, v, r)}^{\prime}}{2 k_{a} \cdot K_{(a, I, v, r)}} .
$$

The only difference between the derivation of this expression and that for the $n$-gluon amplitude given in section 2 is that we must use the modified identity

$$
\frac{1}{\left[K^{2}-m^{2}\right]\left[\left(K+k_{a}\right)^{2}-m^{2}\right]}=\frac{1}{\left[K^{2}-m^{2}\right]\left(2 k_{a} \cdot K\right)}+\frac{1}{\left(-2 k_{a} \cdot K\right)\left[\left(K+k_{a}\right)^{2}-m^{2}\right]}
$$

when gluon $a$ is attached to a propagator of a massive field.

As usual, the color-factor shift associated with gluon $a$ is defined by two requirements: (I) that it satisfy all the algebraic symmetries (e.g., Jacobi relations) obeyed by the color factors $c_{i}^{\prime}$, and (II) that it satisfy

$$
\delta_{a} c_{i}^{\prime} \propto \sum_{c \in S_{a, i}} k_{a} \cdot k_{c}
$$

where $S_{a, i}$ denotes the subset of the external particles on one side of the point at which $a$ is attached to $c_{i}^{\prime}$. These together imply that

$$
\delta_{a} c_{(a, I, v, r)}^{\prime}=\alpha_{(a, I, v)} k_{a} \cdot K_{(a, I, v, r)} .
$$

Therefore the invariance of the amplitude under the color-factor shift implies the constraint

$$
\sum_{I} \sum_{v} \frac{\alpha_{(a, I, v)}}{\prod_{s=1}^{3} d_{(a, I, v, s)}^{\prime}} \sum_{r=1}^{3} n_{(a, I, v, r)}^{\prime}=0
$$

on the sums of kinematic numerators appearing in the cubic decomposition.

As in the case of $n$-gluon amplitudes, color-factor symmetry can be used to derive BCJ relations among the color-ordered amplitudes associated with $\mathcal{A}_{n}$. BCJ relations for $n$-point amplitudes with gluons and a single pair of massive fundamentals were conjectured in ref. [41] and more generally for amplitudes containing an arbitrary number of pairs of fundamentals in ref. [42], based on the assumption of color-kinematic duality. A proof of these BCJ relations using BCFW on-shell recursion was given in ref. [46]. In order to derive these relations, however, it is necessary to write the amplitude in a proper decomposition [45], i.e., in terms of an independent set of color factors and generalizedgauge-invariant primitive amplitudes. For general amplitudes, this is a subtle problem, which was recently solved for the case of multiple pairs of distinct-flavor fundamentals by Melia [43-45] and Johansson and Ochirov [42]. In a sequel to this paper [47], we review their solution, and then derive the BCJ relations using the color-factor symmetry. 
There is one class of amplitudes, however, for which the story is practically identical to the $n$-gluon case, namely, $n$-point amplitudes with $n-2$ gluons and a single pair of fundamentals. For that case, an independent set of $(n-2)$ ! color factors is given by the half-ladders

$$
\mathbf{c}_{1 \gamma n}^{\prime} \equiv\left(T^{\mathrm{a}_{\gamma(2)}} T^{\mathrm{a}_{\gamma(3)}} \cdots T^{\mathrm{a}_{\gamma(n-1)}}\right)^{\mathrm{i}_{1}}{ }_{\mathrm{i}_{n}} .
$$

All other color factors $c_{i}^{\prime}$ can be reduced to half ladders

$$
c_{i}^{\prime}=\sum_{\gamma \in S_{n-2}} M_{i, 1 \gamma n} \mathbf{c}_{1 \gamma n}^{\prime}
$$

by repeatedly applying $f_{\mathrm{abc}}\left(T^{\mathrm{c}}\right)^{\mathrm{i}}{ }_{\mathrm{j}}=\left[T^{\mathrm{a}}, T^{\mathrm{b}}\right]_{\mathrm{j}}^{\mathrm{i}}$, similar to the case of $n$-gluon amplitudes [52]. The coefficients $M_{i, 1 \gamma n}$ are precisely the same as in the $n$-gluon case. The $n$-point amplitude can then be written in a proper decomposition [66, 67]

$$
\mathcal{A}_{n}\left(\bar{\psi}_{1}, g_{2}, g_{3}, \cdots, g_{n-1}, \psi_{n}\right)=\sum_{\gamma \in S_{n-2}} \mathbf{c}_{1 \gamma n}^{\prime} A^{\prime}(1, \gamma(2), \cdots, \gamma(n-1), n)
$$

where the primitive amplitudes are given by

$$
A^{\prime}(1, \gamma(2), \cdots, \gamma(n-1), n)=\sum_{i} \frac{M_{i, 1 \gamma n} n_{i}^{\prime}}{d_{i}^{\prime}}
$$

We define an (n-3)!-parameter family of shifts associated with each gluon $a \in\{2, \cdots, n-1\}$ via

$$
\begin{aligned}
\delta_{a} \mathbf{c}_{1 \sigma(2) \cdots \sigma(b-1) a \sigma(b) \cdots \sigma(n-1) n}^{\prime} & =\alpha_{a, \sigma}\left(k_{a} \cdot k_{1}+\sum_{c=2}^{b-1} k_{a} \cdot k_{\sigma(c)}\right), \quad a, b \in\{2, \cdots, n-1\}, \quad b \neq a \\
\delta_{a} c_{i}^{\prime} & =\sum_{\gamma \in S_{n-2}} M_{i, 1 \gamma n} \delta_{a} \mathbf{c}_{1 \gamma n}^{\prime}
\end{aligned}
$$

where $\gamma$ is a permutation of $\{2, \cdots, n-1\}, \sigma$ is a permutation of $\{2, \cdots, n-1\} \backslash\{a\}$, and $\alpha_{a, \sigma}$ is a set of $(n-3)$ ! arbitrary constants for each $a$. As in the case of the $n$-gluon amplitude, the dimension of the (abelian) group of color-factor shifts is $(n-3)(n-3)$ !. We show in sections 6 and 7 that the amplitude $\mathcal{A}_{n}\left(\bar{\psi}_{1}, g_{2}, g_{3}, \cdots, g_{n-1}, \psi_{n}\right)$ is invariant under the color-factors shifts (5.11). As a consequence, the color-ordered amplitudes defined in eq. (5.9) obey BCJ relations that have the same form (when expressed in terms of invariants $k_{a} \cdot k_{b}$, where $k_{a}$ is the momentum of a gluon) as those for the $n$-gluon amplitude, namely

$$
\sum_{b=3}^{n}\left(k_{1} \cdot k_{2}+\sum_{c=3}^{b-1} k_{2} \cdot k_{\sigma(c)}\right) A^{\prime}(1, \sigma(3), \cdots, \sigma(b-1), 2, \sigma(b), \cdots, \sigma(n-1), n)=0
$$

together with all permutations of this equation with 2 replaced by $a$, as conjectured in refs. [41, 42]. 


\section{Proof of color-factor symmetry for the $\mathcal{A}_{4}\left(\bar{\psi}_{1}, \psi_{2}, g_{3}, g_{4}\right)$ amplitude}

In this section, we prove that the tree-level four-point amplitude with two gluons and two massive particles in an arbitrary representation of the gauge group (which for convenience we refer to as fundamentals) with spin zero, one-half, or one is invariant under the colorfactor symmetry. We will use these results for the proof of the invariance of the more general $n$-point amplitude in section 7 .

The four-point amplitude $\mathcal{A}_{4}\left(\bar{\psi}_{1}, \psi_{2}, g_{3}, g_{4}\right)$ can be constructed from the $\bar{\psi} \psi g$ vertex by attaching a gluon to a propagator emanating from each of the external legs of the vertex, or (in the case of a spin-zero or spin-one fundamental) to the vertex itself. This yields

$$
\mathcal{A}_{4}\left(\bar{\psi}_{1}, \psi_{2}, g_{3}, g_{4}\right)=\sum_{r=1}^{3} \frac{c_{(r)}^{\prime} n_{(r)}^{\prime}}{2 k_{4} \cdot k_{r}}
$$

where the color factors

$$
c_{(1)}^{\prime}=-\left(T^{\mathrm{a}_{4}} T^{\mathrm{a}_{3}}\right)^{\mathrm{i}_{1}}{ }_{\mathrm{i}_{2}}, \quad c_{(2)}^{\prime}=\left(T^{\mathrm{a}_{3}} T^{\mathrm{a}_{4}}\right)^{\mathrm{i}_{1}}{ }_{\mathrm{i}_{2}}, \quad c_{(3)}^{\prime}=f_{\mathrm{a}_{4} \mathrm{a}_{3} \mathrm{~b}}\left(T^{\mathrm{b}}\right)^{\mathrm{i}_{1}}{ }_{\mathrm{i}_{2}}
$$

obey $\sum_{r=1}^{3} c_{(r)}^{\prime}=0$ using $\left[T^{\mathrm{a}}, T^{\mathrm{b}}\right]=f_{\mathrm{abc}} T^{\mathrm{c}}$. The color-factor symmetry (associated with gluon 4) acts on the color factors (6.2) and the four-point amplitude (6.1) as

$$
\delta_{4} c_{(r)}^{\prime}=\alpha_{4} k_{4} \cdot k_{r} \quad \Longrightarrow \quad \delta_{4} \mathcal{A}_{4}=\frac{1}{2} \alpha_{4} \sum_{r=1}^{3} n_{(r)}^{\prime}
$$

We will establish that $\delta_{4} \mathcal{A}_{4}=0$ by showing that $\sum_{r=1}^{3} n_{(r)}^{\prime}=0$, a result that has long been known [23, 24]. We will do this separately for spin zero, spin one-half, and spin one fundamentals.

\subsection{Kinematic numerators for spin-one-half fundamentals}

We begin with the case of a spin-one-half fundamental, which is simpler due to the absence of a $\bar{\psi} \psi g g$ vertex. The $\bar{\psi} \psi g$ vertex $^{13}$ and Dirac propagator are

$$
\frac{i g}{\sqrt{2}}\left(T^{\mathrm{a}_{3}}\right)^{\mathrm{i}_{1}}{ }_{\mathrm{i}_{2}} \gamma^{\mu_{3}}, \quad \frac{i \delta^{\mathrm{i}}{ }_{\mathrm{j}}}{\not k-m} .
$$

Attaching gluon 4 to (fermion) leg 1 yields the expression

$$
-\frac{i g^{2}}{2} \frac{\left(T^{\mathrm{a}_{4}} T^{\mathrm{a}_{3}}\right)^{\mathrm{i}_{1}} \mathrm{i}_{2}}{\left(k_{1}+k_{4}\right)^{2}-m^{2}} \gamma^{\mu_{4}}\left(\not k_{1}+\not k_{4}+m\right) \gamma^{\mu_{3}}=\frac{i g^{2}}{2} \frac{c_{(1)}^{\prime}}{2 k_{4} \cdot k_{1}}\left[\left(-\not k_{1}+m\right) \gamma^{\mu_{4}}+2 k_{1}^{\mu_{4}}+\frac{1}{2}\left[\gamma^{\mu_{4}}, \not k_{4}\right]\right] \gamma^{\mu_{3}} \text {. }
$$

The contribution of this diagram to eq. (6.1) is obtained by sandwiching eq. (6.5) between $\bar{u}\left(k_{1}\right)$ and $u\left(-k_{2}\right)$, contracting with $\varepsilon_{3 \mu_{3}} \varepsilon_{4 \mu_{4}}$, and dividing by $i$. The first term in the square brackets vanishes using $\bar{u}\left(k_{1}\right)\left(-\not k_{1}+m\right)=0$, leaving

$$
n_{(1)}^{\prime}=g^{2} \bar{u}\left(k_{1}\right)\left[\varepsilon_{4} \cdot k_{1}-i \varepsilon_{4 \alpha} k_{4 \beta} \Sigma^{\alpha \beta}\right] \oiint_{3} u\left(-k_{2}\right), \quad \Sigma^{\alpha \beta} \equiv \frac{i}{4}\left[\gamma^{\alpha}, \gamma^{\beta}\right]
$$

\footnotetext{
${ }^{13}$ Recall that $\operatorname{Tr}\left(T^{\mathrm{a}} T^{\mathrm{b}}\right)=\delta^{\mathrm{ab}}$.
} 
where the spin-one-half angular momentum matrices $\Sigma^{\alpha \beta}$ satisfy the Lorentz algebra commutation relations

$$
\left[\Sigma^{\alpha \beta}, \Sigma^{\gamma \delta}\right]=-i\left[\eta^{\alpha \gamma} \Sigma^{\beta \delta}-\eta^{\alpha \delta} \Sigma^{\beta \gamma}-\eta^{\beta \gamma} \Sigma^{\alpha \delta}+\eta^{\beta \delta} \Sigma^{\alpha \gamma}\right]
$$

Similarly, attaching gluon 4 to (fermion) leg 2 yields

$$
-\frac{i g^{2}}{2} \frac{\left(T^{\mathrm{a}_{3}} T^{\mathrm{a}_{4}}\right)^{\mathrm{i}_{1}}{ }_{\mathrm{i}_{2}}}{\left(k_{2}+k_{4}\right)^{2}-m^{2}} \gamma^{\mu_{3}}\left(-\not k_{2}-\not k_{4}+m\right) \gamma^{\mu_{4}}=-\frac{i g^{2}}{2} \frac{c_{(2)}^{\prime}}{2 k_{4} \cdot k_{2}} \gamma^{\mu_{3}}\left[\gamma^{\mu_{4}}\left(k_{2}+m\right)-2 k_{2}^{\mu_{4}}+\frac{1}{2}\left[\gamma^{\mu_{4}}, \not k_{4}\right]\right] \text {. }
$$

Using $\left(\not k_{2}+m\right) u\left(-k_{2}\right)=0$, we obtain

$$
n_{(2)}^{\prime}=g^{2} \bar{u}\left(k_{1}\right) \ddagger_{3}\left[\varepsilon_{4} \cdot k_{2}+i \varepsilon_{4 \alpha} k_{4 \beta} \Sigma^{\alpha \beta}\right] u\left(-k_{2}\right) .
$$

Finally, attaching gluon 4 to (gluon) leg 3 yields

$$
n_{(3)}^{\prime}=g^{2} \varepsilon_{3 \mu_{3}} \bar{u}\left(k_{1}\right)\left[\varepsilon_{4} \cdot k_{3} \delta_{\nu}^{\mu_{3}}-i \varepsilon_{4 \alpha} k_{4 \beta}\left(S_{3}^{\alpha \beta}\right)_{\nu}^{\mu_{3}}\right] \gamma^{\nu} u\left(-k_{2}\right) .
$$

The sum of the kinematic numerators is thus

$$
\begin{aligned}
\sum_{r=1}^{3} n_{(r)}^{\prime}= & g^{2} \bar{u}\left(k_{1}\right) \ddagger_{3} u\left(-k_{2}\right)\left(\sum_{r=1}^{3} \varepsilon_{4} \cdot k_{r}\right) \\
& -i g^{2} \varepsilon_{4 \alpha} k_{4 \beta} \varepsilon_{3 \mu_{3}} \bar{u}\left(k_{1}\right)\left(\Sigma^{\alpha \beta} \gamma^{\mu_{3}}-\gamma^{\mu_{3}} \Sigma^{\alpha \beta}+\left(S_{3}^{\alpha \beta}\right)^{\mu_{3}} \gamma^{\nu}\right) u\left(-k_{2}\right) .
\end{aligned}
$$

The first sum on the right-hand side of this equation vanishes by momentum conservation $\sum_{r=1}^{4} k_{r}=0$ together with $\varepsilon_{4} \cdot k_{4}=0$. The second sum vanishes because

$$
\Sigma^{\alpha \beta} \gamma^{\mu_{3}}-\gamma^{\mu_{3}} \Sigma^{\alpha \beta}+\left(S_{3}^{\alpha \beta}\right)_{\nu}^{\mu_{3}} \gamma^{\nu}
$$

is the first-order Lorentz transformation of $\gamma^{\mu}$ (acting on both spinor indices as well as the vector index) and hence vanishes. Thus the sum of kinematic numerators for the amplitude $\mathcal{A}_{4}\left(\bar{\psi}_{1}, \psi_{2}, g_{3}, g_{4}\right)$ is zero for spin-one-half fundamentals.

\subsection{Kinematic numerators for spin-zero fundamentals}

Next we turn to the case of spin-zero fundamentals. The $\bar{\psi} \psi g$ vertex is

$$
\frac{i g}{\sqrt{2}}\left(T^{\mathrm{a}_{3}}\right)^{\mathrm{i}_{1}}{ }_{\mathrm{i}_{2}} V^{\mu_{3}}\left(k_{1}, k_{2}, k_{3}\right), \quad V^{\mu_{3}}\left(k_{1}, k_{2}, k_{3}\right)=\left(k_{1}-k_{2}\right)^{\mu_{3}}
$$

where $k_{a}$ are outgoing momenta. The scalar propagator is $i \delta^{\mathrm{i}}{ }_{\mathrm{j}} /\left(k^{2}-m^{2}\right)$. Thus attaching gluon 4 to (scalar) leg 1 yields the expression

$$
\begin{gathered}
-\frac{i g^{2}}{2} \frac{\left(T^{\mathrm{a}_{4}} T^{\mathrm{a}_{3}}\right)^{\mathrm{i}_{1}}{ }_{\mathrm{i}_{2}}}{\left(k_{1}+k_{4}\right)^{2}-m^{2}} V^{\mu_{4}}\left(k_{1},-k_{1}-k_{4}, k_{4}\right) V^{\mu_{3}}\left(k_{1}+k_{4}, k_{2}, k_{3}\right) \\
\quad=\frac{i g^{2}}{2} \frac{c^{\prime}}{2 k_{4} \cdot k_{1}}\left[k_{4}^{\mu_{4}}+2 k_{1}^{\mu_{4}}\right] V^{\mu_{3}}\left(k_{1}+k_{4}, k_{2}, k_{3}\right) .
\end{gathered}
$$


The contribution of this diagram to eq. (6.1) is obtained by contracting with $\varepsilon_{3 \mu_{3}} \varepsilon_{4 \mu_{4}}$ and dividing by $i$. The first term in the square brackets vanishes using $\varepsilon_{4} \cdot k_{4}=0$, leaving

$$
\left.n_{(1)}^{\prime}\right|_{\text {leg }}=g^{2} \varepsilon_{4} \cdot k_{1} \varepsilon_{3 \mu_{3}} V^{\mu_{3}}\left(k_{1}+k_{4}, k_{2}, k_{3}\right) .
$$

Similarly, attaching gluon 4 to (scalar) leg 2 yields

$$
\left.n_{(2)}^{\prime}\right|_{\text {leg }}=g^{2} \varepsilon_{4} \cdot k_{2} \varepsilon_{3 \mu_{3}} V^{\mu_{3}}\left(k_{1}, k_{2}+k_{4}, k_{3}\right) .
$$

Attaching gluon 4 to (gluon) leg 3 yields

$$
\left.n_{(3)}^{\prime}\right|_{\operatorname{leg}}=g^{2} \varepsilon_{3 \mu_{3}}\left[\varepsilon_{4} \cdot k_{3} \delta_{\nu}^{\mu_{3}}-i \varepsilon_{4 \alpha} k_{4 \beta}\left(S_{3}^{\alpha \beta}\right)^{\mu_{3}}\right] V^{\nu}\left(k_{1}, k_{2}, k_{3}+k_{4}\right) .
$$

Attaching gluon 4 directly to the $\bar{\psi} \psi g$ vertex, we obtain the $\bar{\psi} \psi g g$ vertex

$$
\frac{i g^{2}}{2} \eta^{\mu_{3} \mu_{4}}\left(\left\{T^{\mathrm{a}_{3}}, T^{\mathrm{a}_{4}}\right\}\right)^{\mathrm{i}_{1}}{ }_{\mathrm{i}_{2}}
$$

which can be written as

$$
-\frac{i g^{2}}{2}\left(c_{(1)}^{\prime} \frac{\partial}{\partial k_{1 \mu_{4}}}+c_{(2)}^{\prime} \frac{\partial}{\partial k_{2 \mu_{4}}}+c_{(3)}^{\prime} \frac{\partial}{\partial k_{3 \mu_{4}}}\right) V^{\mu_{3}}\left(k_{1}, k_{2}, k_{3}\right) .
$$

The rest of the story proceeds exactly as in section 3, allowing us to write the kinematic numerators as

$$
n_{(r)}^{\prime}=g^{2}\left[\varepsilon_{4} \cdot k_{r}-i \varepsilon_{4 \alpha} k_{4 \beta} J_{r}^{\alpha \beta}-i \varepsilon_{4 \alpha} k_{4 \beta} k_{4 \gamma} S_{r}^{\alpha \beta} \frac{\partial}{\partial k_{r \gamma}}\right] V\left(k_{1}, k_{2}, k_{3}\right)
$$

where we have suppressed the polarization vector $\varepsilon_{3}$. Because two of the legs are scalars, we have $S_{1}^{\alpha \beta}=S_{2}^{\alpha \beta}=0$, whereas $S_{3}^{\alpha \beta}$ is given by eq. (3.9).

Now consider the sum of kinematic numerators

$$
\begin{aligned}
\sum_{r=1}^{3} n_{(r)}^{\prime}= & g^{2}\left(\sum_{r=1}^{3} \varepsilon_{4} \cdot k_{r}\right) V\left(k_{1}, k_{2}, k_{3}\right) \\
& -i g^{2} \varepsilon_{4 \alpha} k_{4 \beta}\left(\sum_{r=1}^{3} J_{r}^{\alpha \beta}\right) V\left(k_{1}, k_{2}, k_{3}\right) \\
& -i g^{2} \varepsilon_{4 \alpha} k_{4 \beta} k_{4 \gamma} S_{3}^{\alpha \beta} \frac{\partial}{\partial k_{3 \gamma}} V\left(k_{1}, k_{2}, k_{3}\right)
\end{aligned}
$$

where the scalar-scalar-gluon vertex is $V^{\mu_{3}}\left(k_{1}, k_{2}, k_{3}\right)=\left(k_{1}-k_{2}+\lambda\left[k_{1}+k_{2}+k_{3}\right]\right)^{\mu_{3}}$ with $\lambda$ arbitrary due to momentum conservation. The first sum on the right-hand side of this equation vanishes as usual by momentum conservation. The second sum, which may be written more explicitly as

$$
L_{1}^{\alpha \beta} V^{\mu_{3}}\left(k_{1}, k_{2}, k_{3}\right)+L_{2}^{\alpha \beta} V^{\mu_{3}}\left(k_{1}, k_{2}, k_{3}\right)+\left(J_{3}^{\alpha \beta}\right)_{\nu}^{\mu_{3}} V^{\nu}\left(k_{1}, k_{2}, k_{3}\right)
$$


is the first-order Lorentz transformation of $\bar{\psi} \psi g$ vertex. This vanishes, as may be seen by explicit computation, because $V^{\mu_{3}}\left(k_{1}, k_{2}, k_{3}\right)$ is a Lorentz tensor. The third term on the right-hand side of eq. (6.21) is

$$
\varepsilon_{4 \alpha} k_{4 \beta} k_{4 \gamma}\left(S_{3}^{\alpha \beta}\right)^{\mu_{3}} \frac{\partial}{\partial k_{3 \gamma}} V^{\nu}\left(k_{1}, k_{2}, k_{3}\right)=\lambda\left(k_{4}^{2} \varepsilon_{4}^{\mu_{3}}-\varepsilon_{4} \cdot k_{4} k_{4}^{\mu_{3}}\right)
$$

which automatically vanishes due to $k_{4}^{2}=0$ and $\varepsilon_{4} \cdot k_{4}=0$. Again, we emphasize that in proving the vanishing of eq. (6.22) and (6.23) we did not use that $k_{r}$ were on-shell, nor did we use $\varepsilon_{r} \cdot k_{r}=0$, for $r=1,2,3$. Thus these results remain valid for an off-shell vertex $V^{\mu_{3}}\left(k_{1}, k_{2}, k_{3}\right)$.

\subsection{Kinematic numerators for spin-one fundamentals}

Finally we consider massive spin-one fundamentals with $\bar{\psi} g \psi$ vertex of the form

$$
\frac{i g}{\sqrt{2}}\left(T^{\mathrm{a}_{3}}\right)^{\mathrm{i}_{1}}{ }_{\mathrm{i}_{2}} V^{\mu_{1} \mu_{2} \mu_{3}}\left(k_{1}, k_{2}, k_{3}\right)
$$

where $V^{\mu_{1} \mu_{2} \mu_{3}}\left(k_{1}, k_{2}, k_{3}\right)$ is given by eq. (3.5). We emphasize that although we refer to the vector particles as fundamentals, they could be in any representation, including the adjoint, in which case $\left(T^{\mathrm{a}_{3}}\right)_{\mathrm{a}_{2}}^{\mathrm{a}_{1}}=f_{\mathrm{a}_{1} \mathrm{a}_{3} \mathrm{a}_{2}}$ and eq. (6.24) is equal to eq. (3.4), except that now the vector boson is massive. The propagator for a massive spin-one particle is

$$
\frac{-i \delta_{\mathrm{j}}^{\mathrm{i}} P_{\mu \nu}(k)}{k^{2}-m^{2}}, \quad P_{\mu \nu}(k)=\eta_{\mu \nu}-\frac{k_{\mu} k_{\nu}}{m^{2}} .
$$

In the case in which the vector boson gets its mass from a spontaneously-broken symmetry, eq. (6.25) is the propagator in unitary gauge; this is most convenient for tree-level calculations as we need not compute contributions involving Goldstone bosons.

Attaching gluon 4 to leg 1 yields the expression

$$
\frac{i g^{2}}{2} \frac{c_{(1)}^{\prime}}{\left(k_{1}+k_{4}\right)^{2}-m_{1}^{2}} V^{\mu_{1} \mu_{4} \nu}\left(k_{1}, k_{4},-k_{1}-k_{4}\right) P_{\nu \lambda}\left(k_{1}+k_{4}\right) V^{\lambda \mu_{2} \mu_{3}}\left(k_{1}+k_{4}, k_{2}, k_{3}\right) .
$$

The contribution of this diagram to eq. (6.1) is obtained by contracting with $\prod_{a=1}^{4} \varepsilon_{a \mu_{a}}$ and dividing by $i$, giving

$$
\left.n_{(1)}^{\prime}\right|_{\text {leg }}=g^{2} \varepsilon_{1 \mu_{1}}\left[\varepsilon_{4} \cdot k_{1} \delta_{\nu}^{\mu_{1}}-i \varepsilon_{4 \alpha} k_{4 \beta}\left(S_{1}^{\alpha \beta}\right)^{\mu_{1}}\right] V^{\nu \mu_{2} \mu_{3}}\left(k_{1}+k_{4}, k_{2}, k_{3}\right) \varepsilon_{2 \mu_{2}} \varepsilon_{3 \mu_{3}}
$$

where we have used $0=k_{4}^{2}=\varepsilon_{4} \cdot k_{4}=\varepsilon_{1} \cdot k_{1}$ and $k_{1}^{2}=m_{1}^{2}$ but we did not use eq. (3.7), which is not valid in this case because $k_{2}^{2} \neq k_{3}^{2}$. Analogous expressions are obtained for $n_{(2)}^{\prime}$ and $n_{(3)}^{\prime}$. Attaching gluon 4 directly to the $\bar{\psi} \psi g$ vertex, we obtain the $\bar{\psi} \psi g g$ vertex

$$
-\frac{i g^{2}}{2}\left(c_{(1)}^{\prime} \frac{\partial}{\partial k_{1 \mu_{4}}}+c_{(2)}^{\prime} \frac{\partial}{\partial k_{2 \mu_{4}}}+c_{(3)}^{\prime} \frac{\partial}{\partial k_{3 \mu_{4}}}\right) V^{\mu_{1} \mu_{2} \mu_{3}}\left(k_{1}, k_{2}, k_{3}\right) .
$$

Again, the rest of the story proceeds exactly as in section 3, allowing us to write the kinematic numerators as

$$
n_{(r)}^{\prime}=g^{2}\left[\varepsilon_{4} \cdot k_{r}-i \varepsilon_{4 \alpha} k_{4 \beta} J_{r}^{\alpha \beta}-i \varepsilon_{4 \alpha} k_{4 \beta} k_{4 \gamma} S_{r}^{\alpha \beta} \frac{\partial}{\partial k_{r \gamma}}\right] V\left(k_{1}, k_{2}, k_{3}\right)
$$


where we have suppressed the polarization vectors $\varepsilon_{r}$ for $r=1,2,3$. Note that the kinematic numerators $n_{(r)}^{\prime}$ have exactly the same form as the kinematic numerators for the four-gluon amplitude, even though the masses for particles 1 through 3 can be nonzero. The proof of the vanishing of the sum of numerators proceeds exactly as in section 3 .

In this section, we have explicitly shown that the sum of kinematic numerators for four-point amplitudes $\mathcal{A}_{4}\left(\bar{\psi}_{1}, \psi_{2}, g_{3}, g_{4}\right)$ vanishes (where $\psi$ can have spin zero, one-half, or one) and thus have demonstrated the invariance of the four-point amplitude under the color-factor symmetry associated with gluon 4. As the results of the last subsection have shown, this result remains valid even when particles 1 through 3 are massive; only the gluon associated with the color-factor symmetry need be massless. The results we have derived will be used in the next section to prove a more general result.

\section{Proof of color-factor symmetry for more general amplitudes}

In this section, we use the radiation vertex expansion to demonstrate the invariance under the color-factor symmetry of tree-level gauge-theory amplitudes containing at least one gluon together with massless or massive particles in arbitrary representations of the gauge group (but referred to as fundamentals for convenience) and with arbitrary spin $\leq 1$. For concreteness, we focus on the $n$-point amplitude $\mathcal{A}_{n}\left(\bar{\psi}_{1}, g_{2}, g_{3}, \cdots, g_{n-1}, \psi_{n}\right)$ with $n-$ 2 gluons and a pair of fundamentals $\psi$, but it will be clear that the proof applies to more general amplitudes. The proof is very similar to that given in section 4 for $n$-gluon amplitudes, and so we only highlight the differences.

The radiation vertex expansion constructs the $n$-point amplitude $\mathcal{A}_{n}\left(\bar{\psi}_{1}, g_{2}, g_{3}, \cdots, g_{n-1}, \psi_{n}\right)$ by attaching gluon $a \in\{2, \cdots, n-1\}$ to all possible $(n-1)$-point diagrams $I^{\prime}$, with two fundamentals and $n-3$ gluons, in all possible ways, and reorganizing this as a sum over all the vertices of the $(n-1)$-point diagram. We have already shown in section 4 that the contributions of the three- and four-gluon vertices are invariant under the color-factor symmetry, so we only need to demonstrate the same for vertices involving two fundamentals. We do this separately for fundamentals with spin zero, one-half, and one.

\subsection{Vertices involving spin-one-half fundamentals}

To derive the contribution of the $\bar{\psi} \psi g$ vertices to the radiation vertex expansion for spinone-half fundamentals $\psi$, we examine the effect of attaching a gluon to a fermion leg, either external or internal.

First we single out (fermion) leg 1 , denoting the contribution of an $(n-1)$-point diagram $I^{\prime}$ to the amplitude as $\bar{u}\left(k_{1}\right) C^{\mathrm{i}_{1}}\left(k_{1}, \cdots\right)$ where $\cdots$ denotes momenta belonging to $\{2, \cdots, n\} \backslash\{a\}$. Attaching gluon $a$ to external fermion leg 1 , using $\bar{u}\left(k_{1}\right)\left(-\not k_{1}+m\right)=0$, and contracting with $\varepsilon_{a \mu_{a}}$, we obtain

$$
-\sqrt{2} g \frac{\left(T^{\mathrm{a}_{a}}\right)^{\mathrm{i}_{1}} \mathrm{j}}{2 k_{a} \cdot k_{1}} \bar{u}\left(k_{1}\right)\left[\varepsilon_{a} \cdot k_{1}-i \varepsilon_{a \alpha} k_{a \beta} \Sigma^{\alpha \beta}\right] C^{\mathrm{j}}\left(k_{1}+k_{a}, \cdots\right) .
$$

Next, we single out (fermion) leg $n$, denoting the contribution of the diagram $I^{\prime}$ to the amplitude as $B_{\mathrm{i}_{n}}\left(k_{n}, \cdots\right) u\left(-k_{n}\right)$, where $\cdots$ denotes momenta belonging to $\{1, \cdots, n-1\} \backslash$ 
$\{a\}$. Attach gluon $a$ to external fermion leg $n$, use $\left(\not k_{n}+m\right) u\left(-k_{n}\right)=0$, and contract with $\varepsilon_{a \mu_{a}}$ to obtain

$$
+\sqrt{2} g B_{\mathrm{j}}\left(k_{n}+k_{a}, \cdots\right) \frac{\left(T^{\mathrm{a}_{a}}\right)^{\mathrm{j}}{ }_{\mathrm{i}_{n}}}{2 k_{a} \cdot k_{n}}\left[\varepsilon_{4} \cdot k_{n}+i \varepsilon_{4 \alpha} k_{4 \beta} \Sigma^{\alpha \beta}\right] u\left(-k_{n}\right) .
$$

Now we single out one of the internal fermion lines of $I^{\prime}$, which divides the diagram into two subdiagrams $B_{\mathrm{j}}$ and $C^{\mathrm{j}}$, and splits the external legs $\{1, \cdots, n\} \backslash\{a\}$ into two complementary sets $S_{a, B}$ and $S_{a, C}$. The contribution of the diagram $I^{\prime}$ can thus be written as

$$
B_{\mathrm{j}}(-K, \cdots) \frac{i \delta_{\mathrm{k}}^{\mathrm{j}}}{\not K-m} C^{\mathrm{k}}(K, \cdots)
$$

where $K=\sum_{d \in S_{a, B}} k_{d}$ is the momentum running through the line, and the $\cdots$ in $B$ and $C$ denote momenta belonging to $S_{a, B}$ and $S_{a, C}$ respectively. Attaching gluon $a$ to the line connecting the two subgraphs and contracting with $\varepsilon_{a \mu_{a}}$, we have

$$
-\frac{i g}{\sqrt{2}} B_{\mathrm{j}}(-K, \cdots) \frac{1}{\not K-m}\left(T^{\mathrm{a}_{a}}\right)^{\mathrm{j}}{ }_{\mathrm{k}}{ }_{a} \frac{1}{\not K+\not k_{a}-m} C^{\mathrm{k}}\left(K+k_{a}, \cdots\right) .
$$

Now we use the identity [35]

$$
\frac{1}{\not K-m} \ddagger_{a} \frac{1}{\not K+\not k_{a}-m}=\frac{1}{\not K-m} \frac{\left(\varepsilon_{a} \cdot K+\frac{1}{4}\left[\phi_{a}, \not k_{a}\right]\right)}{k_{a} \cdot K}-\frac{\left(\varepsilon_{a} \cdot K+\frac{1}{4}\left[\phi_{a}, \not k_{a}\right]\right)}{k_{a} \cdot K} \frac{1}{\not K+\not k_{a}-m}
$$

to rewrite this as

$$
\begin{aligned}
& B_{\mathrm{j}}(-K, \cdots) \frac{i}{\not K-m}\left\{-\sqrt{2} g \frac{\left(T^{\mathrm{a}_{a}}\right)_{\mathrm{k}}^{\mathrm{j}}}{2 k_{a} \cdot K}\left[\varepsilon_{a} \cdot K-i \varepsilon_{a \alpha} k_{a \beta} \Sigma^{\alpha \beta}\right] C^{\mathrm{k}}\left(K+k_{a}, \cdots\right)\right\} \\
& +\left\{\sqrt{2} g \frac{\left(T^{\mathrm{a}_{a}}\right)^{\mathrm{j}}{ }_{\mathrm{k}}}{2 k_{a} \cdot K} B_{\mathrm{j}}(-K, \cdots)\left[\varepsilon_{a} \cdot K-i \varepsilon_{a \alpha} k_{a \beta} \Sigma^{\alpha \beta}\right]\right\} \frac{i}{\not K+\not k_{a}-m} C^{\mathrm{k}}\left(K+k_{a}, \cdots\right) .
\end{aligned}
$$

Each term can be associated with one of the two vertices to which the line is attached.

We now choose one of the $\bar{\psi} \psi g$ vertices $v$ of $I^{\prime}$. Such a vertex divides the external legs into three non-overlapping subsets $S_{\left(a, I^{\prime}, v, r\right)}, r=1,2,3$ such that $\bigcup_{r=1}^{3} S_{\left(a, I^{\prime}, v, r\right)}=$ $\{1, \cdots, n\} \backslash\{a\}$. The contribution of the diagram $I^{\prime}$ to the $(n-1)$-point amplitude can be expressed as

$$
\frac{i g}{\sqrt{2}} A_{\mathrm{c}_{3} \mu_{3}}^{(3)}\left(-K_{3}, \cdots\right) B_{\mathrm{j}_{1}}\left(-K_{1}, \cdots\right) \gamma^{\mu_{3}}\left(T^{\mathrm{c}_{3}}\right)^{\mathrm{j}_{1}}{ }_{\mathrm{j}_{2}} C^{\mathrm{j}_{2}}\left(-K_{2}, \cdots\right)
$$

where $K_{r}=\sum_{d \in S_{\left(a, I^{\prime}, v, r\right)}} k_{d}$ is the momentum flowing out of each leg of the vertex, and the $\cdots$ in $B, C$, and $A$ denote momenta belonging to $S_{\left(a, I^{\prime}, v, 1\right)}, S_{\left(a, I^{\prime}, v, 2\right)}$, and $S_{\left(a, I^{\prime}, v, 3\right)}$ respectively. If either fermion leg is external, then $B_{\mathrm{j}_{1}}=\bar{u}\left(k_{1}\right) \delta^{\mathrm{i}_{1}}{ }_{\mathrm{j}_{1}}$ or $C^{\mathrm{j}_{2}}=u\left(-k_{n}\right) \delta^{\mathrm{j}_{2}}{ }_{{ }_{i_{n}}}$. If the gluon leg is external, then $A_{\mathrm{c}_{3} \mu_{3}}^{(3)}$ is $\delta_{\mathrm{bc}_{3}} \varepsilon_{b \mu_{b}}$. 
We now attach gluon $a$ to each of the legs of this $\bar{\psi} \psi g$ vertex, either to an external leg or to an internal line. Using the expressions above as well as those in section 4, we obtain

$$
\begin{aligned}
& i g^{2} A_{\mathrm{c}_{3} \mu_{3}}^{(3)}\left(-K_{3}, \cdots\right) B_{\mathrm{j}_{1}}\left(-K_{1}, \cdots\right) \\
& \times\left(-\frac{\left(T^{\mathrm{a}_{a}} T^{\mathrm{c}_{3}}\right)^{\mathrm{j}_{1}} \mathrm{j}_{2}}{2 k_{a} \cdot K_{1}}\left[\varepsilon_{a} \cdot K_{1}-i \varepsilon_{a \alpha} k_{a \beta} \Sigma^{\alpha \beta}\right] \gamma^{\mu_{3}}+\frac{\left(T^{\mathrm{c}_{3}} T^{\mathrm{a}_{a}}\right)^{\mathrm{j}_{1}}{ }_{\mathrm{j}_{2}}}{2 k_{a} \cdot K_{2}} \gamma^{\mu_{3}}\left[\varepsilon_{a} \cdot K_{2}+i \varepsilon_{a \alpha} k_{a \beta} \Sigma^{\alpha \beta}\right]\right. \\
& \left.\quad+\frac{f_{\mathrm{a}_{a} \mathrm{c}_{3} \mathrm{~b}}\left(T^{\mathrm{b}}\right)^{\mathrm{j}_{1}} \mathrm{j}_{2}}{2 k_{a} \cdot K_{3}}\left[\eta^{\mu_{3} \nu} \varepsilon_{a} \cdot K_{3}-i \varepsilon_{a \alpha} k_{a \beta}\left(S_{3}^{\alpha \beta}\right)^{\mu_{3} \nu}\right] \gamma_{\nu}\right) C^{\mathrm{j}_{2}}\left(-K_{2}, \cdots\right)
\end{aligned}
$$

which is the contribution of the $\bar{\psi} \psi g$ vertex to the radiation vertex expansion.

We now wish to show that eq. (7.8) is invariant under the color-factor symmetry. As in section 4 , we first assume that the subdiagrams corresponding to $A, B$, and $C$ contain no four-gluon vertices. Designate by $c_{\left(a, I^{\prime}, v, r\right)}$ with $r=1,2,3$ the color factors associated with each of the three terms in eq. (7.8), including factors of $f_{\mathrm{abc}}$ and $\left(T^{\mathrm{a}}\right)_{\mathrm{k}}^{\mathrm{j}}$ in the subdiagrams. These color factors manifestly satisfy $\sum_{r=1}^{3} c_{\left(a, I^{\prime}, v, r\right)}=0$, and the variation of $c_{\left(a, I^{\prime}, v, r\right)}$ under the color-factor shift associated with gluon $a$ is

$$
\delta_{a} c_{\left(a, I^{\prime}, v, r\right)}=\alpha_{\left(a, I^{\prime}, v\right)} k_{a} \cdot K_{r}
$$

which preserves $\sum_{r=1}^{3} c_{\left(a, I^{\prime}, v, r\right)}=0$. The variation of eq. (7.8) under eq. (7.9) is then proportional to

$$
\left(\sum_{r=1}^{3} \varepsilon_{a} \cdot K_{r}\right) \gamma^{\mu_{3}}-i \varepsilon_{a \alpha} k_{a \beta}\left[\Sigma^{\alpha \beta} \gamma^{\mu_{3}}-\gamma^{\mu_{3}} \Sigma^{\alpha \beta}+\left(S_{3}^{\alpha \beta}\right)^{\mu_{3} \nu} \gamma_{\nu}\right]
$$

The first term vanishes by momentum conservation, and the second by the transformation properties of $\gamma_{\nu}$, as we saw in section 6 . If the subdiagrams $A, B$, and $C$ do contain fourgluon vertices, we can expand eq. (7.8) into individual pieces, each of which is invariant under the color-factor symmetry. Together with the result from section 4 that the contributions to the radiation vertex expansion from the three- and four-gluon vertices are also invariant, we have thus shown that the amplitude $\mathcal{A}_{n}\left(\bar{\psi}_{1}, g_{2}, g_{3}, \cdots, g_{n-1}, \psi_{n}\right)$ with spinone-half fundamentals is invariant under the color-factor symmetry. In fact, this proof applies to an amplitude with an arbitrary number of pairs of fundamentals, and will be used in the sequel [47] to prove the BCJ relations [42] for that class of amplitudes.

\subsection{Vertices involving spin-zero fundamentals}

To derive the contribution of the $\bar{\psi} \psi g$ and $\bar{\psi} \psi g g$ vertices to the radiation vertex expansion for spin-zero fundamentals $\psi$, we examine the effect of attaching a gluon to a scalar leg, either external or internal.

First we single out (scalar) leg 1, denoting the contribution of an $(n-1)$-point diagram $I^{\prime}$ to the amplitude as $C^{\mathrm{i}_{1}}\left(k_{1}, \cdots\right)$ where $\cdots$ denotes momenta belonging to $\{2, \cdots, n\} \backslash\{a\}$. 
Attaching gluon $a$ to external scalar leg 1 we obtain

$$
\begin{gathered}
-\frac{g}{\sqrt{2}} \frac{\left(T^{\mathrm{a}_{a}}\right)^{\mathrm{i}_{1}}{ }_{\mathrm{j}}}{\left(k_{a}+k_{1}\right)^{2}-m^{2}} V^{\mu_{a}}\left(k_{1},-k_{1}-k_{a}, k_{a}\right) C^{\mathrm{j}}\left(k_{1}+k_{a}, \cdots\right) \\
=-\frac{g}{\sqrt{2}} \frac{\left(T^{\mathrm{a}_{a}}\right)^{\mathrm{i}_{1}}{ }_{\mathrm{j}}}{2 k_{a} \cdot k_{1}}\left[k_{a}^{\mu_{a}}+2 k_{1}^{\mu_{a}}\right] C^{\mathrm{j}}\left(k_{1}+k_{a}, \cdots\right) .
\end{gathered}
$$

Contracting this with $\varepsilon_{a \mu_{a}}$ eliminates the first term in the square brackets, leaving

$$
-\sqrt{2} g \frac{\left(T^{\mathrm{a}_{a}}\right)^{\mathrm{i}_{1}} \mathrm{j}}{2 k_{a} \cdot k_{1}} \varepsilon_{a} \cdot k_{1} C^{\mathrm{j}}\left(k_{1}+k_{a}, \cdots\right) .
$$

Similarly, attaching gluon $a$ to external scalar leg $n$, we obtain

$$
+\sqrt{2} g \varepsilon_{a} \cdot k_{n} B_{\mathrm{j}}\left(k_{n}+k_{a}, \cdots\right) \frac{\left(T^{\mathrm{a}_{a}}\right)^{\mathrm{j}} \mathrm{i}_{n}}{2 k_{a} \cdot k_{n}} .
$$

Next we single out one of the internal scalar lines of $I^{\prime}$, which divides the diagram into two subdiagrams $B_{\mathrm{j}}$ and $C^{\mathrm{j}}$, and splits the external legs into two complementary sets $S_{a, B}$ and $S_{a, C}$. The contribution of the diagram $I^{\prime}$ can thus be written as

$$
B_{\mathrm{j}}(-K, \cdots) \frac{i \delta_{\mathrm{k}}^{\mathrm{j}}}{K^{2}-m^{2}} C^{\mathrm{k}}(K, \cdots)
$$

where $K=\sum_{d \in S_{a, B}} k_{d}$ is the momentum running through the line, and the $\cdots$ in $B$ and $C$ denote momenta belonging to $S_{a, B}$ and $S_{a, C}$ respectively. Attaching gluon $a$ to the line connecting the two subgraphs yields

$$
-\frac{i g}{\sqrt{2}} B_{\mathrm{j}}(-K, \cdots) \frac{\left(T^{\mathrm{a}_{a}}\right)^{\mathrm{j}}{ }_{\mathrm{k}} V^{\mu_{a}}\left(K,-K-k_{a}, k_{a}\right)}{\left[K^{2}-m^{2}\right]\left[\left(K+k_{a}\right)^{2}-m^{2}\right]} C^{\mathrm{k}}\left(K+k_{a}, \cdots\right) .
$$

Contracting with $\varepsilon_{a \mu_{a}}$ and using $\varepsilon_{a} \cdot k_{a}=0$, we have

$$
-\sqrt{2} i g B_{\mathrm{j}}(-K, \cdots) \frac{\left(T^{\mathrm{a}_{a}}\right)_{\mathrm{k}}^{\mathrm{j}} \varepsilon_{a} \cdot K}{\left[K^{2}-m^{2}\right]\left[\left(K+k_{a}\right)^{2}-m^{2}\right]} C^{\mathrm{k}}\left(K+k_{a}, \cdots\right) .
$$

Now we use the identity (5.3) to rewrite this as

$$
\begin{aligned}
& B_{\mathrm{j}}(-K, \cdots) \frac{i}{K^{2}-m^{2}}\left\{-\sqrt{2} g \frac{\left(T^{\mathrm{a}_{a}}\right)_{\mathrm{k}}^{\mathrm{j}}}{2 k_{a} \cdot K} \varepsilon_{a} \cdot K C^{\mathrm{k}}\left(K+k_{a}, \cdots\right)\right\} \\
& +\left\{\sqrt{2} g B_{\mathrm{j}}(-K, \cdots) \frac{\left(T^{\mathrm{a}_{a}}\right)^{\mathrm{j}}{ }_{\mathrm{k}}}{2 k_{a} \cdot K} \varepsilon_{a} \cdot K\right\} \frac{i}{\left(K+k_{a}\right)^{2}-m^{2}} C^{\mathrm{k}}\left(K+k_{a}, \cdots\right) .
\end{aligned}
$$

We associate each of the terms in this equation with one of the two vertices to which the line is attached.

Next we choose one of the scalar-scalar-gluon vertices $v$ of $I^{\prime}$ (if it has any). Such a vertex divides the external legs into three non-overlapping subsets $S_{\left(a, I^{\prime}, v, r\right)}, r=1,2,3$ 
such that $\bigcup_{r=1}^{3} S_{\left(a, I^{\prime}, v, r\right)}=\{1, \cdots, n\} \backslash\{a\}$. The contribution of the diagram $I^{\prime}$ can be expressed as

$$
\frac{i g}{\sqrt{2}} V^{\mu_{3}}\left(K_{1}, K_{2}, K_{3}\right) A_{\mathrm{c}_{3} \mu_{3}}^{(3)}\left(-K_{3}, \cdots\right) B_{\mathrm{j}_{1}}\left(-K_{1}, \cdots\right)\left(T^{\mathrm{c}_{3}}\right)^{\mathrm{j}_{1}}{ }_{\mathrm{j}_{2}} C^{\mathrm{j}_{2}}\left(-K_{2}, \cdots\right)
$$

where $K_{r}=\sum_{d \in S_{\left(a, I^{\prime}, v, r\right)}} k_{d}$ is the momentum flowing out of each leg of the vertex, and the $\cdots$ in $B, C$, and $A$ denote momenta belonging to $S_{\left(a, I^{\prime}, v, 1\right)}, S_{\left(a, I^{\prime}, v, 2\right)}$, and $S_{\left(a, I^{\prime}, v, 3\right)}$ respectively. If either scalar leg is external, then $B_{\mathrm{j}_{1}}=\delta^{\mathrm{i}_{1}}{ }_{\mathrm{j}_{1}}$ or $C^{\mathrm{j}_{2}}=\delta^{\mathrm{j}_{2}}{ }_{{ }_{\mathrm{i}}}$. If the gluon leg is external, then $A_{\mathrm{c}_{3} \mu_{3}}^{(3)}$ is $\delta_{\mathrm{bc}_{3}} \varepsilon_{b \mu_{b}}$.

We now attach gluon $a$ to each of the legs of this scalar-scalar-gluon vertex, either to an external leg or to an internal line. This yields

$$
\begin{aligned}
& i g^{2} A_{\mathrm{c}_{3} \mu_{3}}^{(3)}\left(-K_{3}, \cdots\right) B_{\mathrm{j}_{1}}\left(-K_{1}, \cdots\right) \\
& \times\left(-\frac{\left(T^{\mathrm{a}_{a}} T^{\mathrm{c}_{3}}\right)^{\mathrm{j}_{1}} \mathrm{j}_{2}}{2 k_{a} \cdot K_{1}}\left[\varepsilon_{a} \cdot K_{1}\right] V^{\mu_{3}}\left(K_{1}+k_{a}, K_{2}, K_{3}\right)+\frac{\left(T^{\mathrm{c}_{3}} T^{\mathrm{a}_{a}}\right)^{\mathrm{j}_{1}} \mathrm{j}_{2}}{2 k_{a} \cdot K_{2}}\left[\varepsilon_{a} \cdot K_{2}\right] V^{\mu_{3}}\left(K_{1}, K_{2}+k_{a}, K_{3}\right)\right. \\
& \left.\quad+\frac{f_{\mathrm{a}_{a} \mathrm{c}_{3} \mathrm{~b}}\left(T^{\mathrm{b}}\right)^{\mathrm{j}_{1}} \mathrm{j}_{2}}{2 k_{a} \cdot K_{3}}\left[\eta^{\mu_{3} \nu} \varepsilon_{a} \cdot K_{3}+\left(\varepsilon_{a}^{\mu_{3}} k_{a}^{\nu}-\varepsilon_{a}^{\nu} k_{a}^{\mu_{3}}\right)\right] V_{\nu}\left(K_{1}, K_{2}, K_{3}+k_{a}\right)\right) C^{\mathrm{j}_{2}}\left(-K_{2}, \cdots\right) .
\end{aligned}
$$

We can also attach gluon $a$ directly to the scalar-scalar-gluon vertex itself. Using eq. (6.19), this yields

$$
\begin{aligned}
& -\frac{i g^{2}}{2} A_{\mathrm{c}_{3} \mu_{3}}^{(3)}\left(-K_{3}, \cdots\right) B_{\mathrm{j}_{1}}\left(-K_{1}, \cdots\right) \\
& \quad \times\left(-\left(T^{\mathrm{a}_{a}} T^{\mathrm{c}_{3}}\right)^{\mathrm{j}_{1}}{ }_{\mathrm{j}_{2}} \varepsilon_{a \mu_{a}} \frac{\partial}{\partial K_{1 \mu_{a}}} V^{\mu_{3}}\left(K_{1}, K_{2}, K_{3}\right)+\left(T^{\mathrm{c}_{3}} T^{\mathrm{a}_{a}}\right)^{\mathrm{j}_{1}}{ }_{\mathrm{j}_{2}} \varepsilon_{a \mu_{a}} \frac{\partial}{\partial K_{2 \mu_{a}}} V^{\mu_{3}}\left(K_{1}, K_{2}, K_{3}\right)\right. \\
& \left.\quad+f_{\mathrm{a}_{a} \mathrm{c}_{3} \mathrm{~b}}\left(T^{\mathrm{b}}\right)^{\mathrm{j}_{1}}{ }_{\mathrm{j}_{2}} \varepsilon_{a \mu_{a}} \frac{\partial}{\partial K_{3 \mu_{a}}} V^{\mu_{3}}\left(K_{1}, K_{2}, K_{3}\right)\right) C^{\mathrm{j}_{2}}\left(-K_{2}, \cdots\right) .
\end{aligned}
$$

We now use eq. (3.16) in eq. (7.19), and combine eq. (7.19) and (7.20) as we did in section 6. Leaving the $\mu_{3}$ index implicit, we obtain the contribution of the scalar-scalar-gluon vertex to the radiation vertex expansion

$$
\begin{aligned}
& i g^{2} A_{\mathrm{c}_{3}}^{(3)}\left(-K_{3}, \cdots\right) B_{\mathrm{j}_{1}}\left(-K_{1}, \cdots\right) \\
& \times\left(-\frac{\left(T^{\mathrm{a}_{a}} T^{\mathrm{c}_{3}}\right)^{\mathrm{j}_{1}} \mathrm{j}_{2}}{2 k_{a} \cdot K_{1}}\left[\varepsilon_{a} \cdot K_{1}-i \varepsilon_{a \alpha} k_{a \beta} L_{1}^{\alpha \beta}\right] V\left(K_{1}, K_{2}, K_{3}\right)\right. \\
& \quad+\frac{\left(T^{\mathrm{c}_{3}} T^{\mathrm{a}_{a}}\right)^{\mathrm{j}_{1}} \mathrm{j}_{2}}{2 k_{a} \cdot K_{2}}\left[\varepsilon_{a} \cdot K_{2}-i \varepsilon_{a \alpha} k_{a \beta} L_{2}^{\alpha \beta}\right] V\left(K_{1}, K_{2}, K_{3}\right) \\
& \left.\quad+\frac{f_{\mathrm{a}_{a} \mathrm{c}_{3} \mathrm{~b}}\left(T^{\mathrm{b}}\right)^{\mathrm{j}_{1}} \mathrm{j}_{2}}{2 k_{a} \cdot K_{3}}\left[\varepsilon_{a} \cdot K_{3}-i \varepsilon_{a \alpha} k_{a \beta} J_{3}^{\alpha \beta}-i \varepsilon_{a \alpha} k_{a \beta} k_{a \gamma} S_{3}^{\alpha \beta} \frac{\partial}{\partial K_{3 \gamma}}\right] V\left(K_{1}, K_{2}, K_{3}\right)\right) C^{\mathrm{j}_{2}}\left(-K_{2}, \cdots\right)
\end{aligned}
$$

where $J_{3}$ and $S_{3}$ act on the $\mu_{3}$ index of $V\left(K_{1}, K_{2}, K_{3}\right)$.

At the end of the last subsection, we discussed the color-factor symmetry acting on the $\bar{\psi} \psi g$ vertex contribution to the radiation vertex expansion. Under eq. (7.9), the variation of eq. (7.21) is proportional to

$$
\left[\left(\sum_{r=1}^{3} \varepsilon_{a} \cdot K_{r}\right)-i \varepsilon_{a \alpha} k_{a \beta}\left(\sum_{r=1}^{3} J_{r}^{\alpha \beta}\right)-i \varepsilon_{a \alpha} k_{a \beta} k_{a \gamma} S_{3}^{\alpha \beta} \frac{\partial}{\partial K_{3 \gamma}}\right] V\left(K_{1}, K_{2}, K_{3}\right) .
$$


In section 6 , we demonstrated that each of the three terms in eq. (7.22) vanishes. Therefore the contributions of the $\bar{\psi} \psi g$ vertices (7.21) to the radiation vertex expansion are invariant under the color-factor shift associated with gluon $a$.

Finally we choose one of the scalar-scalar-gluon-gluon vertices $v$ of $I^{\prime}$ (if it has any). Such a vertex divides the external legs into four non-overlapping subsets $S_{\left(a, I^{\prime}, v, r\right)}, r=$ $1, \cdots, 4$ such that $\bigcup_{r=1}^{4} S_{\left(a, I^{\prime}, v, r\right)}=\{1, \cdots, n\} \backslash\{a\}$. Using eq. (6.18), the contribution of diagram $I^{\prime}$ can be expressed as

$$
\frac{i g^{2}}{2} \eta^{\mu_{3} \mu_{4}} A_{\mathrm{c}_{3} \mu_{3}}^{(3)}\left(-K_{3}, \cdots\right) A_{\mathrm{c}_{4} \mu_{4}}^{(4)}\left(-K_{4}, \cdots\right) B_{\mathrm{j}_{1}}\left(-K_{1}, \cdots\right)\left(\left\{T^{\mathrm{c}_{3}}, T^{\mathrm{c}_{4}}\right\}\right)^{\mathrm{j}_{1}}{ }_{\mathrm{j}_{2}} C^{\mathrm{j}_{2}}\left(-K_{2}, \cdots\right) .
$$

We now attach gluon $a$ to each of the legs of this scalar-scalar-gluon-gluon vertex, either to an external leg or to an internal line. This yields

$$
\begin{aligned}
& \frac{i g^{3}}{\sqrt{2}} A_{\mathrm{c}_{3} \mu_{3}}^{(3)}\left(-K_{3}, \cdots\right) A_{\mathrm{c}_{4} \mu_{4}}^{(4)}\left(-K_{4}, \cdots\right) B_{\mathrm{j}_{1}}\left(-K_{1}, \cdots\right) \\
& \times\left(-\frac{\left(T^{\mathrm{a}_{a}}\left\{T^{\mathrm{c}_{3}}, T^{\mathrm{c}_{4}}\right\}\right)^{\mathrm{j}_{1}} \mathrm{j}_{2}}{2 k_{a} \cdot K_{1}}\left[\varepsilon_{a} \cdot K_{1}\right] \eta^{\mu_{3} \mu_{4}}+\frac{\left(\left\{T^{\mathrm{c}_{3}}, T^{\mathrm{c}_{4}}\right\} T^{\mathrm{a}_{a}}\right)^{\mathrm{j}_{1}} \mathrm{j}_{2}}{2 k_{a} \cdot K_{2}}\left[\varepsilon_{a} \cdot K_{2}\right] \eta^{\mu_{3} \mu_{4}}\right. \\
& \quad+\frac{f_{\mathrm{a}_{a} \mathrm{c}_{3} \mathrm{~b}}\left(\left\{T^{\mathrm{b}}, T^{\mathrm{c}_{4}}\right\}\right)^{\mathrm{j}_{1}} \mathrm{j}_{2}}{2 k_{a} \cdot K_{3}}\left[\delta^{\mu_{3}} \varepsilon_{a} \cdot K_{3}-i \varepsilon_{a \alpha} k_{a \beta}\left(S_{3}^{\alpha \beta}\right)^{\mu_{3}}{ }_{\nu}\right] \eta^{\nu \mu_{4}} \\
& \left.\quad+\frac{f_{\mathrm{a}_{a} \mathrm{c}_{4} \mathrm{~b}}\left(\left\{T^{\mathrm{c}_{3}}, T^{\mathrm{b}}\right\}\right)^{\mathrm{j}_{1}} \mathrm{j}_{2}}{2 k_{a} \cdot K_{4}}\left[\delta_{\nu}^{\mu_{4}} \varepsilon_{a} \cdot K_{4}-i \varepsilon_{a \alpha} k_{a \beta}\left(S_{4}^{\alpha \beta}\right)^{\mu_{4}}\right] \eta^{\mu_{3} \nu}\right) C^{\mathrm{j}_{2}}\left(-K_{2}, \cdots\right) .
\end{aligned}
$$

We now need to consider the variation under the color-factor symmetry of this contribution to the radiation vertex expansion. Designate by $c_{\left(a, I^{\prime}, v, r\right)}$ with $r=1, \cdots 4$ the color factor associated with each of the terms in eq. (7.24), including the factors of $f_{\mathrm{abc}}$ and $\left(T^{\mathrm{a}}\right)_{\mathrm{k}}^{\mathrm{j}}$ in the subdiagrams. These color factors satisfy $\sum_{r=1}^{4} c_{\left(a, I^{\prime}, v, r\right)}=0$ by virtue of

$$
-\left(T^{\mathrm{a}_{a}}\left\{T^{\mathrm{c}_{3}}, T^{\mathrm{c}_{4}}\right\}\right)^{\mathrm{j}_{1}} \mathrm{j}_{2}+\left(\left\{T^{\mathrm{c}_{3}}, T^{\mathrm{c}_{4}}\right\} T^{\mathrm{a}_{a}}\right)^{\mathrm{j}_{1}}{ }_{\mathrm{j}_{2}}+f_{\mathrm{a}_{a} \mathrm{c}_{3} \mathrm{~b}}\left(\left\{T^{\mathrm{b}}, T^{\mathrm{c}_{4}}\right\}\right)^{\mathrm{j}_{1}}{ }_{\mathrm{j}_{2}}+f_{\mathrm{a}_{a} \mathrm{c}_{4} \mathrm{~b}}\left(\left\{T^{\mathrm{c}_{3}}, T^{\mathrm{b}}\right\}\right)^{\mathrm{j}_{1}} \mathrm{j}_{2}=0 .
$$

The variation of $c_{\left(a, I^{\prime}, v, r\right)}$ under the color-factor shift associated with gluon $a$ is

$$
\delta_{a} c_{\left(a, I^{\prime}, v, r\right)}=\alpha_{\left(a, I^{\prime}, v\right)} k_{a} \cdot K_{r} .
$$

The variation of eq. (7.24) under eq. (7.26) is therefore proportional to

$$
\left(\sum_{r=1}^{4} \varepsilon_{a} \cdot K_{r}\right) \eta^{\mu_{3} \mu_{4}}-i \varepsilon_{a \alpha} k_{a \beta}\left[\left(S_{3}^{\alpha \beta}\right)_{\nu}^{\mu_{3}} \eta^{\nu \mu_{4}}+\left(S_{4}^{\alpha \beta}\right)^{\mu_{4}} \eta^{\mu_{3} \nu}\right] .
$$

The first term vanishes by momentum conservation, $k_{a}+\sum_{r=1}^{4} K_{r}=0$, and $\varepsilon_{a} \cdot k_{a}=0$. The second term is the first-order Lorentz transformation of the tensor $\eta^{\mu_{3} \mu_{4}}$, which vanishes.

Thus each vertex involving scalars that contributes to the radiation vertex expansion is invariant under the color-factor shift associated with gluon $a$. Together with the result from section 4 that the contributions from the three- and four-gluon vertices to the radiation vertex expansion are also invariant, we have shown that the full amplitude 
$\mathcal{A}_{n}\left(\bar{\psi}_{1}, g_{2}, g_{3}, \cdots, g_{n-1}, \psi_{n}\right)$ with spin-zero fundamentals is invariant under the color-factor shift. In fact, any amplitude built with $\bar{\psi} \psi g$ and $\bar{\psi} \psi g g$ vertices for scalar $\psi$ will have the color-factor symmetry.

\subsection{Vertices involving spin-one fundamentals}

To derive the contribution of the $\bar{\psi} \psi g$ and $\bar{\psi} \psi g g$ vertices to the radiation vertex expansion for spin-one fundamentals $\psi$, we examine the effect of attaching a gluon to a massive vector leg, either external or internal.

First we single out (vector) leg 1 , denoting the contribution of an $(n-1)$-point diagram $I^{\prime}$ to the amplitude as $\varepsilon_{1 \mu_{1}} C^{\mathrm{i}_{1} \mu_{1}}\left(k_{1}, \cdots\right)$ where $\cdots$ denotes momenta belonging to $\{2, \cdots, n\} \backslash\{a\}$. Attaching gluon $a$ to external vector leg 1 we obtain

$$
-\frac{g}{\sqrt{2}} \frac{\left(T^{\mathrm{a}_{a}}\right)^{\mathrm{i}_{1}}{ }_{\mathrm{j}}}{\left(k_{a}+k_{1}\right)^{2}-m_{1}^{2}} V^{\mu_{1} \nu \mu_{a}}\left(k_{1},-k_{1}-k_{a}, k_{a}\right) P_{\nu \lambda}\left(k_{1}+k_{a}\right) C^{\mathrm{j} \lambda}\left(k_{1}+k_{a}, \cdots\right) .
$$

Contracting with $\varepsilon_{1 \mu_{1}} \varepsilon_{a \mu_{a}}$, we obtain

$$
-\sqrt{2} g \frac{\left(T^{\mathrm{a}_{a}}\right)^{\mathrm{i}_{1}} \mathrm{j}}{2 k_{a} \cdot k_{1}} \varepsilon_{1 \mu_{1}}\left[\varepsilon_{a} \cdot k_{1} \eta^{\mu_{1} \nu}+\left(\varepsilon_{a}^{\mu_{1}} k_{a}^{\nu}-\varepsilon_{a}^{\nu} k_{a}^{\mu_{1}}\right)\right] C_{\nu}^{\mathrm{j}}\left(k_{1}+k_{a}, \cdots\right)
$$

where we used $k_{4}^{2}=\varepsilon_{4} \cdot k_{4}=\varepsilon_{1} \cdot k_{1}=0$ and $k_{1}^{2}=m_{1}^{2}$. We did not use the vanishing of eq. (4.3). Similar expressions result from attaching gluon $a$ to the other legs. By comparing eq. (4.4) and (7.29), we observe that the expression is the same for a massless or a massive vector particle.

Next we single out one of the internal lines of $I^{\prime}$, which divides the diagram into two subdiagrams $B$ and $C$, and splits the external legs $\{1, \cdots, n\} \backslash\{a\}$ into two complementary sets $S_{a, B}$ and $S_{a, C}$. The contribution of the diagram can thus be written as

$$
B_{\mathrm{j}}^{\mu}(-K, \cdots) \frac{\left(-i \delta_{\mathrm{k}}^{\mathrm{j}}\right) P_{\mu \nu}(K)}{K^{2}-m^{2}} C^{\mathrm{k} \nu}(K, \cdots)
$$

where $K=\sum_{d \in S_{a, B}} k_{d}$ is the momentum running through the line, and the $\cdots$ in $B$ and $C$ denote momenta belonging to $S_{a, B}$ and $S_{a, C}$ respectively. Attaching gluon $a$ to the line connecting the two subgraphs and contracting with $\varepsilon_{a \mu_{a}}$, we obtain

$-\frac{i g}{\sqrt{2}} B_{\mathrm{j}}^{\mu}(-K, \cdots) \frac{\left(T^{\mathrm{a}_{a}}\right)_{\mathrm{k}}^{\mathrm{j}} P_{\mu \lambda}(K) \varepsilon_{a \mu_{a}} V^{\lambda \kappa \mu_{a}}\left(K,-K-k_{a}, k_{a}\right) P_{\kappa \nu}\left(K+k_{a}\right)}{\left[K^{2}-m^{2}\right]\left[\left(K+k_{a}\right)^{2}-m^{2}\right]} C^{\mathrm{k} \nu}\left(K+k_{a}, \cdots\right)$.

Now we use the identity [35]

$$
\begin{aligned}
& \frac{P_{\mu \lambda}(K) \varepsilon_{a \mu_{a}} V^{\lambda \kappa \mu_{a}}\left(K,-K-k_{a}, k_{a}\right) P_{\kappa \nu}\left(K+k_{a}\right)}{\left[K^{2}-m^{2}\right]\left[\left(K+k_{a}\right)^{2}-m^{2}\right]} \\
& =\frac{1}{k_{a} \cdot K}\left\{\frac{-P_{\mu \lambda}(K)\left[\delta_{\nu}^{\lambda} \varepsilon_{a} \cdot K+\varepsilon_{a}^{\lambda} k_{a \nu}-k_{a}^{\lambda} \varepsilon_{a \nu}\right]}{K^{2}-m^{2}}+\frac{\left[\delta_{\mu}{ }^{\kappa} \varepsilon_{a} \cdot K+\varepsilon_{a \mu} k_{a}^{\kappa}-k_{a \mu} \varepsilon_{a}^{\kappa}\right] P_{\kappa \nu}\left(K+k_{a}\right)}{\left(K+k_{a}\right)^{2}-m^{2}}\right\}
\end{aligned}
$$

to rewrite this as 


$$
\begin{aligned}
& B_{\mathrm{j}}^{\mu}(-K, \cdots) \frac{-i P_{\mu \lambda}(K)}{K^{2}-m^{2}}\left\{-\sqrt{2} g \frac{\left(T^{\mathrm{a}_{a}}\right)_{\mathrm{k}}^{\mathrm{j}}}{2 k_{a} \cdot K}\left[\eta^{\lambda \nu} \varepsilon_{a} \cdot K+\left(\varepsilon_{a}^{\lambda} k_{a}^{\nu}-\varepsilon_{a}^{\nu} k_{a}^{\lambda}\right)\right] C_{\nu}^{\mathrm{k}}\left(K+k_{a}, \cdots\right)\right\} \\
& +\left\{\sqrt{2} g B_{\mathrm{j} \mu}(\cdots,-K) \frac{\left(T^{\mathrm{a} a}\right)^{\mathrm{j}} \mathrm{k}}{2 k_{a} \cdot K}\left[\eta^{\mu \kappa} \varepsilon_{a} \cdot K+\left(\varepsilon_{a}^{\mu} k_{a}^{\kappa}-\varepsilon_{a}^{\kappa} k_{a}^{\mu}\right)\right]\right\} \frac{-i P_{\kappa \nu}\left(K+k_{a}\right)}{\left(K+k_{a}\right)^{2}-m^{2}} C^{\mathrm{k} \nu}\left(K+k_{a}, \cdots\right) .
\end{aligned}
$$

We did not use the vanishing of eq. (4.8). We associate each of the terms in curly brackets with one of the two vertices to which the line is attached.

For the rest of the discussion, we can be brief. The expressions for the contributions to the radiation vertex expansion from $\bar{\psi} \psi g$ and $\bar{\psi} \psi g g$ vertices are similar to those for spin-zero fundamentals, except that we must include $J_{r}$ and $S_{r}$ terms for $r=1$ and 2 . The proof that these vertex contributions are invariant under the color-factor symmetry relies on some of the results from section 4 .

Thus we have shown that the full amplitude $\mathcal{A}_{n}\left(\bar{\psi}_{1}, g_{2}, g_{3}, \cdots, g_{n-1}, \psi_{n}\right)$ with massive spin-one fundamentals is invariant under the color-factor shift. In fact, any amplitude built with $\bar{\psi} \psi g$ and $\bar{\psi} \psi g g$ vertices with a massive vector particle $\psi$ will have the colorfactor symmetry.

We can go even further and state that any amplitude built from $g g g$ and $\bar{\psi} g \psi$ vertices (with $\psi$ having arbitrary spin $\leq 1$ ) where not only $\psi$ but also some of the gluons are massive (i.e., through spontaneous symmetry breaking) will be invariant under the color-factor shift. The only particle in the amplitude that must be massless is the gluon associated with the color-factor symmetry.

\section{Null eigenvectors of the propagator matrix}

The symmetry that we have introduced in this paper is possessed not only by gauge-theory amplitudes but also by the amplitudes of the much simpler theory [48] of massless scalars $\phi^{\text {aa' }}$ transforming in the adjoint of the color group $\mathrm{U}(N) \times \mathrm{U}(\tilde{N})$. These bi-adjoint scalars have only cubic interactions of the form

$$
f_{\mathrm{abc}} \tilde{f}_{\mathrm{a}^{\prime} \mathrm{b}^{\prime} \mathrm{c}^{\prime}} \phi^{\mathrm{aa}^{\prime}} \phi^{\mathrm{bb}^{\prime}} \phi^{\mathrm{cc} \mathrm{c}^{\prime}}
$$

where $f_{\mathrm{abc}}$ and $\tilde{f}_{\mathrm{a}^{\prime} \mathrm{b}^{\prime} \mathrm{c}^{\prime}}$ are the structure constants of $\mathrm{U}(N)$ and $\mathrm{U}(\tilde{N})$. The tree-level $n$-point amplitude is given by the sum over cubic diagrams

$$
\mathcal{A}_{n}^{\text {scalar }}=\sum_{i} \frac{c_{i} \tilde{c}_{i}}{d_{i}}
$$

Using eq. (2.10), the bi-adjoint scalar tree amplitude (8.2) can be written as

$$
\mathcal{A}_{n}^{\text {scalar }}=\sum_{\gamma \in S_{n-2}} \sum_{\delta \in S_{n-2}} \mathbf{c}_{1 \gamma n} m(1 \gamma n \mid 1 \delta n) \tilde{\mathbf{c}}_{1 \delta n}
$$

where

$$
m(1 \gamma n \mid 1 \delta n)=\sum_{i} \frac{M_{i, 1 \gamma n} M_{i, 1 \delta n}}{d_{i}}
$$


are double-partial amplitudes of the bi-adjoint scalar theory [48]. The $m(1 \gamma n \mid 1 \delta n)$ are also the entries of $(n-2) ! \times(n-2)$ ! propagator matrix defined in ref. [7]. Vaman and Yao argued that the propagator matrix has rank $(n-3)$ ! by virtue of momentum conservation, using explicit low $n$ examples. Cachazo, He, and Yuan confirmed this for general $n$ by expressing the double-partial amplitudes as a sum over the $(n-3)$ ! solutions of the scattering equations [48].

The cubic vertex expansion of the $n$-point bi-adjoint scalar amplitude with respect to external scalar $a$ (see section 2) is given by

$$
\mathcal{A}_{n}^{\text {scalar }}=\sum_{I} \sum_{v \in V_{(a, I)}} \frac{1}{\prod_{s=1}^{3} d_{(a, I, v, s)}} \sum_{r=1}^{3} \frac{c_{(a, I, v, r)} \tilde{c}_{(a, I, v, r)}}{2 k_{a} \cdot K_{(a, I, v, r)}}
$$

where

$$
\sum_{r=1}^{3} c_{(a, I, v, r)}=0, \quad \quad \sum_{r=1}^{3} \tilde{c}_{(a, I, v, r)}=0 .
$$

The color-factor shift with respect to massless scalar $a$ acts on the color factors appearing in eq. (8.5) as

$$
\delta_{a} c_{(a, I, v, r)}=\alpha_{(a, I, v)} k_{a} \cdot K_{(a, I, v, r)} .
$$

The variation of eq. (8.5) under this shift

$$
\delta_{a} \mathcal{A}_{n}^{\text {scalar }}=\frac{1}{2} \sum_{I} \sum_{v \in V_{(a, I)}} \frac{\alpha_{(a, I, v)}}{\prod_{s=1}^{3} d_{(a, I, v, s)}} \sum_{r=1}^{3} \tilde{c}_{(a, I, v, r)}
$$

vanishes by virtue of eq. (8.6), thus establishing that the amplitudes of the bi-adjoint scalar theory possess the color-factor symmetry.

Now we consider the variation of the amplitude (8.3) under the shift (2.14) associated with $a=2$,

$$
\begin{aligned}
& \delta_{2} \mathcal{A}_{n}^{\text {scalar }} \\
& \left.=\sum_{\sigma \in S_{n-3}} \alpha_{2, \sigma} \sum_{b=3}^{n}\left(k_{1} \cdot k_{2}+\sum_{c=3}^{b-1} k_{2} \cdot k_{\sigma(c)}\right) m(1, \sigma(3), \cdots, \sigma(b-1), 2, \sigma(b), \cdots, \sigma(n-1), n \mid 1 \delta n)\right) \tilde{\mathbf{c}}_{1 \delta n} .
\end{aligned}
$$

Using the invariance of $\mathcal{A}_{n}^{\text {scalar }}$, together with independence of $\alpha_{2, \sigma}$ and $\tilde{\mathbf{c}}_{1 \delta n}$, we obtain

$$
\sum_{b=3}^{n}\left(k_{1} \cdot k_{2}+\sum_{c=3}^{b-1} k_{2} \cdot k_{\sigma(c)}\right) m(1, \sigma(3), \cdots, \sigma(b-1), 2, \sigma(b), \cdots, \sigma(n-1), n \mid 1 \delta n)=0
$$

i.e., we have derived a set of $(n-3)$ ! null eigenvectors of the propagator matrix. Other sets of null eigenvectors are obtained from the color-factor shifts associated the other massless scalars in the amplitude.

Since the $(n-2) ! \times(n-2)$ ! propagator matrix is known [48] to have rank $(n-3)$ !, at most $(n-3)(n-3)$ ! of these null eigenvectors can be independent. Thus, the color-factor symmetry associated with $n-3$ of the massless scalars suffices to guarantee the reduced 
rank of the propagator matrix. This nicely explains a result found by one of the present authors in ref. [68], viz., that the propagator matrix persists in having rank $(n-3)$ ! even when up to three of the external particles in the amplitude are massive, but if more than three particles are massive, the rank of the propagator matrix is greater. We now see that for $m \geq 3$ massive and $n-m$ massless particles, the number of null eigenvectors generated by the color-factor symmetry will be $(n-m)(n-3)$ ! , and therefore the rank of the propagator matrix will be $(m-2)(n-3)$ ! for $m=3$ through $m=n$.

Returning now to Yang-Mills theory, if we assume that the numerators of the $n$-gluon amplitude obey color-kinematic duality, i.e. they obey the same Jacobi relations as $c_{i}$, then they can similarly be expressed in terms of $(n-2)$ ! half-ladder numerators $\mathbf{n}_{1 \gamma n}$ via

$$
n_{i}=\sum_{\gamma \in S_{n-2}} M_{i, 1 \gamma n} \mathbf{n}_{1 \gamma n} .
$$

Using eq. (2.17) and (8.11), the color-ordered amplitudes in the Kleiss-Kuijf basis can be written in terms of the propagator matrix as

$$
A(1, \gamma(2), \cdots, \gamma(n-1), n)=\sum_{\delta \in S_{n-2}} m(1 \gamma n \mid 1 \delta n) \mathbf{n}_{1 \delta n} .
$$

Thus the null eigenvectors of the propagator matrix (8.10) imply that the color-ordered $n$-gluon amplitudes obey the BCJ relations (2.19).

As we saw in section 2, however, it is not necessary to require color-kinematic duality in order to prove the BCJ relations. The BCJ relations follow from the weaker constraint (2.26), and both eq. (2.26) and the BCJ relations are a consequence of the color-factor symmetry of the amplitude, which is established through the radiation vertex expansion.

\section{Loop-level amplitudes}

Given the connection established in this paper between the color-factor symmetry of treelevel gauge-theory amplitudes and color-kinematic duality/BCJ relations, it is naturally of great interest to see whether these ideas can be extended to loop level. In this section, we generalize the cubic vertex expansion introduced in section 2 to loop-level amplitudes. We then define a set of shifts of one-loop color factors that depend on the loop momentum as well as the momenta of external particles, and ask whether the cubic vertex expansion of the one-loop amplitude is invariant under these shifts.

It is obvious from the variation of the cubic vertex expansion (2.25) that the treelevel amplitude will be invariant under a color-factor shift if the numerators satisfy colorkinematic duality. The reader may have noticed, however, that up until now we have taken great pains not to invoke color-kinematic duality to prove color-factor symmetry. We have chosen rather to show that it follows directly from a Lagrangian approach. At loop level, we no longer have that luxury, at least at the current stage of development. Instead we will explicitly invoke loop-level color-kinematic duality (for the theories in which it has been shown to hold $[1,2,10-20])$ in order to demonstrate the color-factor symmetry of the 


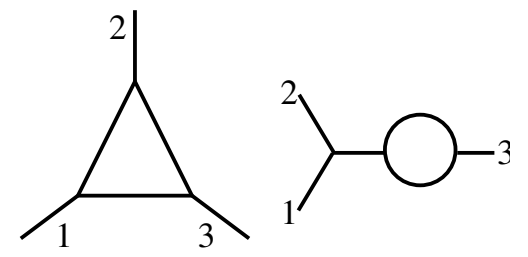

Figure 4. Some of the diagrams to which a gluon is attached to obtain the one-loop four-point cubic decomposition.
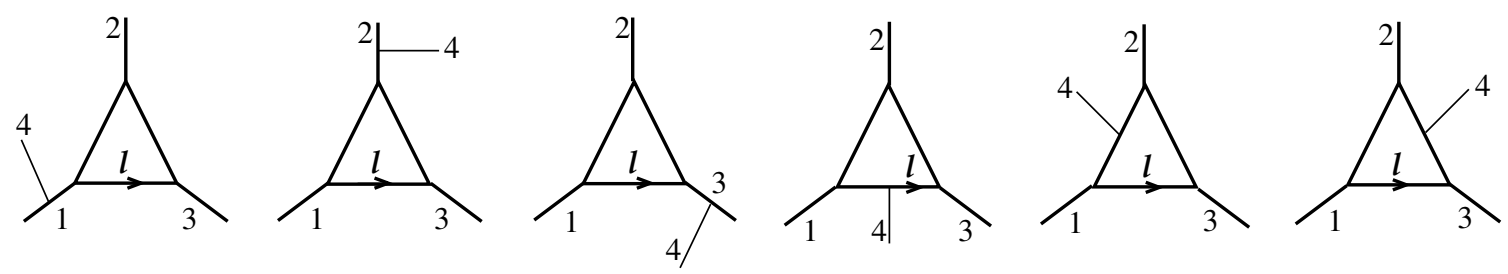

Figure 5. Diagrams [14]23, 1[24]3, 12[34], 1234, 1423, and 1243.

one-loop amplitude. We then show that the invariance of the amplitude under color-factor shifts implies a set of relations among the integrands of its color-ordered amplitudes.

\subsection{Cubic vertex expansion for loop-level amplitudes}

To construct the cubic vertex expansion of an $L$-loop $n$-gluon amplitude with respect to gluon $a$, we begin with the set of $L$-loop $(n-1)$-point cubic diagrams $I$ with external legs $\{1, \cdots, n\} \backslash\{a\}$. For example, for the one-loop four-gluon amplitude, two of the three-point diagrams are shown in figure 4 ; the rest are obtained from relabelings of the external legs. Then we attach gluon $a$ in all possible ways, either to the external legs or to the internal lines of $I$. For example, attaching gluon 4 to the triangle diagram in figure 4 in all possible ways, we obtain the diagrams in figure 5 . The diagrams in figure 5 correspond to the following terms in the cubic decomposition of the one-loop four-point amplitude $[1,2]$

$$
\begin{aligned}
\mathcal{A}_{4, \mathrm{tri}}^{(1)}=\int \frac{d^{D} \ell}{(2 \pi)^{D}} & {\left[\frac{c_{[14] 23} n_{[14] 23}}{d_{[14] 23}}+\frac{c_{1[24] 3} n_{1[24] 3}}{d_{1[24] 3}}+\frac{c_{12[34]} n_{12[34]}}{d_{12[34]}}\right.} \\
& \left.+\frac{c_{1234} n_{1234}}{d_{1234}}+\frac{c_{1423} n_{1423}}{d_{1423}}+\frac{c_{1243} n_{1243}}{d_{1243}}\right]
\end{aligned}
$$

where the denominators in eq. (9.1) are the products of inverse propagators associated with the diagrams. (There could be different sets of denominators depending on the mass of the particle circulating in the loop.) Explicit definitions of the color factors are given below in eq. (9.6). The terms obtained by attaching the gluon to an internal line - in this case, the last three terms of eq. (9.1) - are split into two by applying the identity (2.20) or, in the case of massive internal lines, eq. (5.3). The terms are then reorganized into a sum over the vertices of $I$. For example, the terms in eq. (9.1) are reorganized into 


$$
\begin{aligned}
\mathcal{A}_{4, \mathrm{tri}}^{(1)}= & \int \frac{d^{D} \ell}{(2 \pi)^{D}}\left\{\frac{1}{\ell^{2}\left(\ell-k_{2}-k_{3}\right)^{2}\left(\ell-k_{3}\right)^{2}}\left[\frac{c_{[14] 23} n_{[14] 23}}{2 k_{4} \cdot k_{1}}+\frac{c_{1234} n_{1234}}{2 k_{4} \cdot \ell}-\frac{c_{1423} n_{1423}}{2 k_{4} \cdot\left(\ell+k_{1}\right)}\right]\right. \\
& +\frac{1}{\ell^{2}\left(\ell+k_{1}\right)^{2}\left(\ell-k_{3}\right)^{2}}\left[\frac{c_{1[24] 3} n_{1[24] 3}}{2 k_{4} \cdot k_{2}}+\frac{c_{1423} n_{1423}}{2 k_{4} \cdot\left(\ell+k_{1}\right)}-\frac{c_{1243} n_{1243}}{2 k_{4} \cdot\left(\ell+k_{1}+k_{2}\right)}\right] \\
& \left.+\frac{1}{\ell^{2}\left(\ell+k_{1}\right)^{2}\left(\ell+k_{1}+k_{2}\right)^{2}}\left[\frac{c_{12[34]} n_{12[34]}}{2 k_{4} \cdot k_{3}}+\frac{c_{1243} n_{1243}}{2 k_{4} \cdot\left(\ell+k_{1}+k_{2}\right)}-\frac{c_{1234} n_{1234}}{2 k_{4} \cdot\left(\ell+k_{1}+k_{2}+k_{3}\right)}\right]\right\}
\end{aligned}
$$

where $\ell$ in the last term of the last line of eq. (9.2) differs from the label in figure 5 by a shift. ${ }^{14}$ If the particle circulating in the loop has mass $m$, the expressions $(\ell+\cdots)^{2}$ in eq. (9.2) (i.e., those outside the square brackets) are all replaced by $(\ell+\cdots)^{2}-m^{2}$. We hasten to remind the reader that $\mathcal{A}_{4, \text { tri }}^{(1)}$ is only one part of the one-loop four-gluon amplitude; similar expressions are obtained by attaching gluon 4 to the other one-loop three-point diagrams $I$.

Observe that if we apply a shift to the numerators

$$
\begin{aligned}
& \delta_{4} n_{[14] 23}=\beta k_{4} \cdot k_{1}, \quad \delta_{4} n_{1[24] 3}=\beta k_{4} \cdot k_{2}, \quad \delta_{4} n_{12[34]}=\beta k_{4} \cdot k_{3}, \\
& \delta_{4} n_{1234}=\beta k_{4} \cdot \ell, \quad \delta_{4} n_{1423}=\beta k_{4} \cdot\left(\ell+k_{1}\right), \quad \delta_{4} n_{1243}=\beta k_{4} \cdot\left(\ell+k_{1}+k_{2}\right),
\end{aligned}
$$

the expression (9.2) remains unchanged as a result of the Jacobi identities

$$
0=c_{[14] 23}+c_{1234}-c_{1423}=c_{1[24] 3}+c_{1423}-c_{1243}=c_{12[34]}+c_{1243}-c_{1234}
$$

which means that eq. (9.3) corresponds to a generalized gauge transformation.

The $n$-point generalization of eq. (9.2), obtained by attaching gluon $n$ to the $(n-1)$-gon diagram, is given by

$$
\begin{aligned}
\mathcal{A}_{n,(n-1) \operatorname{gon}}^{(1)} & =\frac{d^{D} \ell}{(2 \pi)^{D}} \sum_{b=1}^{n-1}\left\{\frac{1}{\prod_{c=1}^{b}\left(\ell+\sum_{a=1}^{c-1} k_{a}\right)^{2} \prod_{d=b+1}^{n-1}\left(\ell-\sum_{a=d}^{n-1} k_{a}\right)^{2}}\right. \\
& \left.\times\left[\frac{c_{\ldots[b n] \cdots n \ldots[b n] \cdots}}{2 k_{n} \cdot k_{b}}+\frac{c_{\cdots b-1, n, b \cdots n \ldots b-1, n, b \cdots}}{2 k_{n} \cdot\left(\ell+\sum_{a=1}^{b-1} k_{a}\right)}-\frac{c_{\cdots b, n, b+1 \ldots n \ldots b, n, b+1 \cdots}}{2 k_{n} \cdot\left(\ell+\sum_{a=1}^{b} k_{a}\right)}\right]\right\}
\end{aligned}
$$

where

$$
\begin{aligned}
c_{12 \cdots n} & \equiv \sum_{\mathrm{b}_{1}, \ldots, \mathrm{b}_{n}} f_{\mathrm{b}_{1} \mathrm{a}_{1} \mathrm{~b}_{2}} f_{\mathrm{b}_{2} \mathrm{a}_{2} \mathrm{~b}_{3}} \cdots f_{\mathrm{b}_{n} \mathrm{a}_{n} \mathrm{~b}_{1}}, \\
c_{[12] 3 \cdots n} & \equiv \sum_{\mathrm{b}_{1}, \ldots, \mathrm{b}_{n}} f_{\mathrm{a}_{1} \mathrm{a}_{2} \mathrm{~b}_{2}} f_{\mathrm{b}_{1} \mathrm{~b}_{2} \mathrm{~b}_{3}} f_{\mathrm{b}_{3} \mathrm{a}_{3} \mathrm{~b}_{3}} \cdots f_{\mathrm{b}_{n} \mathrm{a}_{n} \mathrm{~b}_{1}} .
\end{aligned}
$$

\footnotetext{
${ }^{14}$ This points up an inherent ambiguity in defining a common loop momentum when adding together different loop-level diagrams, as in eq. (9.1) and (9.2). This requires further study. For now, we simply note that there exists a choice of loop momentum for each diagram such that (part of) the one-loop amplitude has the form (9.2). With this choice, the numerator shifts defined in eq. (9.3) correspond to a generalized gauge transformation.
} 

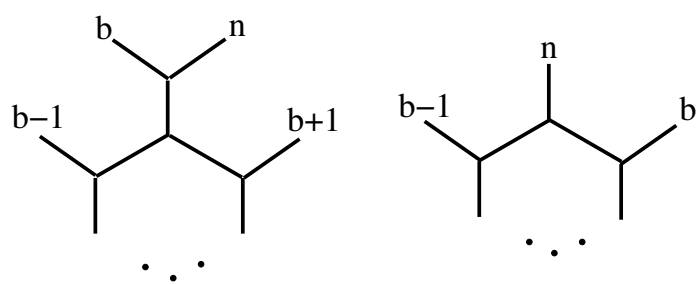

Figure 6. Diagrams with color factors $c_{\ldots[b n] \cdots}$ and $c_{\ldots b-1, n, b \cdots}$

The "ring" diagrams $c_{12 \cdots n}$ are cyclically symmetric and reflection symmetric

$$
c_{12 \ldots n}=(-1)^{n} c_{n \cdots 21} .
$$

The color factors appearing in eq. (9.5) are shown in figure 6 and satisfy the Jacobi relations

$$
0=c_{\ldots[b n] \cdots}+c_{\ldots b-1, n, b \cdots}-c_{\ldots b, n, b+1 \cdots}
$$

Again, if a particle of mass $m$ is circulating in the loop, we replace terms of the form $\left(\ell+\sum k\right)^{2}$ with $\left(\ell+\sum k\right)^{2}-m^{2}$. Similar expressions are obtained by attaching gluon $n$ to the other one-loop $(n-1)$-point diagrams $I$, including $(n-1)$-gon diagrams with other permutations of the external legs $\{1, \cdots, n-1\}$,

Recently, partial fraction identities similar to eq. (2.20) have been employed to recast one-loop amplitudes into a new form whose denominators contain factors linear in the loop momentum [69-74], somewhat analogous to eq. (9.5). These new expressions are those that naturally emerge from a scattering-equation approach to loop-level amplitudes.

\subsection{Color-factor symmetry at one-loop level}

Next we consider the behavior of one-loop amplitudes under momentum-dependent shifts of its color factors. First we must define a set of shifts consistent with the requirements elucidated earlier in the paper. For the one-loop four-gluon amplitude, the color-factor shift associated with gluon 4 must satisfy

$$
\delta_{4} c_{[14] 23}=\alpha k_{4} \cdot k_{1}, \quad \delta_{4} c_{1[24] 3}=\alpha k_{4} \cdot k_{2}, \quad \delta_{4} c_{12[34]}=\alpha k_{4} \cdot k_{3}
$$

since these diagrams have gluon 4 attached to an external leg. Requiring the color-factor shift to respect the Jacobi relations (9.4) implies that

$$
\delta_{4} c_{1423}=\delta_{4} c_{1234}+\alpha k_{4} \cdot k_{1} \quad \delta_{4} c_{1243}=\delta_{4} c_{1234}+\alpha k_{4} \cdot\left(k_{1}+k_{2}\right) .
$$

Unlike the tree-level case, however, these requirements alone are not sufficient to fix the values of all the shifts; one of them, $\delta_{4} c_{1234}$, remains arbitrary. In analogy with eq. (9.3), we define the remaining shift to be

$$
\delta_{4} c_{1234}=\alpha k_{4} \cdot \ell
$$


where $\ell$ is the loop momentum. ${ }^{15}$ The effect of this shift on eq. (9.2) is

$$
\begin{gathered}
\delta_{4} \mathcal{A}_{4, \text { tri }}^{(1)}=\frac{\alpha}{2} \int \frac{d^{D} \ell}{(2 \pi)^{D}}\left[\frac{n_{[14] 23}+n_{1234}-n_{1423}}{\ell^{2}\left(\ell-k_{2}-k_{3}\right)^{2}\left(\ell-k_{3}\right)^{2}}+\frac{n_{1[24] 3}+n_{1423}-n_{1243}}{\ell^{2}\left(\ell+k_{1}\right)^{2}\left(\ell-k_{3}\right)^{2}}\right. \\
\left.+\frac{n_{12[34]}+n_{1243}-n_{1234}}{\ell^{2}\left(\ell+k_{1}\right)^{2}\left(\ell+k_{1}+k_{2}\right)^{2}}\right] .
\end{gathered}
$$

Similar expressions are obtained for the contributions to the cubic vertex expansion from the other three-point diagrams.

A goal consistent with the development in this paper would be to prove by some alternative means (such as the radiation vertex expansion) that $\delta_{4} \mathcal{A}_{4}^{(1)}$ vanishes under the one-loop color-factor shift. That would imply the vanishing of the r. h. s. of eq. (9.12) plus the expressions obtained from the other three-point diagrams, imposing a generalizedgauge-invariant constraint on the one-loop kinematic numerators (namely, that the terms in the square brackets in eq. (9.12) add up to something that integrates to zero). But at this point in the development of the subject, we have no such proof, and therefore we will turn the argument around, and use the knowledge that there exist kinematic numerators for the one-loop four-gluon amplitude that obey color-kinematic duality

$$
0=n_{[14] 23}+n_{1234}-n_{1423}=n_{1[24] 3}+n_{1423}-n_{1243}=n_{12[34]}+n_{1243}-n_{1234}
$$

for pure Yang-Mills theory (with only gluons circulating in the loop) [19] as well as for theories with other particles circulating in the loop [1,20]. In these cases, the kinematic Jacobi identities (9.13) imply that eq. (9.12) vanishes, as do the other contributions to the cubic vertex expansion. Thus, the one-loop four-gluon amplitude in these theories is invariant under the color-factor shift specified by eqs. (9.9), (9.10), and (9.11).

The cubic vertex expansion of the one-loop four-point amplitude of the bi-adjoint scalar theory may be obtained by replacing the kinematic numerators $n_{i}$ with a second copy of the color factors $\tilde{c}_{i}$. Since the latter obey the one-loop color Jacobi identities (9.4), the biadjoint scalar one-loop four-point amplitude is also invariant under the color-factor shift.

It is known that an independent basis of one-loop color factors are those associated with ring diagrams modulo cyclic permutations and reflections [52]. Therefore the one-loop $n$-gluon amplitude can be written

$$
\mathcal{A}_{n}^{(1)}=\int \frac{d^{D} \ell}{(2 \pi)^{D}} \sum_{\gamma \in S_{n-1} / \mathbb{Z}_{2}} c_{1 \gamma} I(1, \gamma(2), \cdots, \gamma(n))
$$

where $\gamma$ is a permutation of $\{2, \cdots, n\}, \mathbb{Z}_{2}$ denotes the reflection symmetry $c_{123 \ldots n} \rightarrow$ $c_{1 n \cdots 32}$, and $I(1, \gamma(2), \cdots, \gamma(n))$ are the integrands of the one-loop color-ordered amplitudes. Eq. (9.14) may be regarded as the result of a generalized gauge transformation in which the kinematic numerators associated with the non-ring color factors are set to zero [11]. Specializing to the one-loop four-gluon amplitude, eq. (9.14) gives

$$
\mathcal{A}_{4}^{(1)}=\int \frac{d^{D} \ell}{(2 \pi)^{D}}\left[c_{1234} I(1,2,3,4)+c_{1423} I(1,4,2,3)+c_{1243} I(1,2,4,3)\right] .
$$

\footnotetext{
${ }^{15}$ See footnote 14 .
} 
For theories whose numerators respect one-loop color-kinematic duality, the invariance of the one-loop amplitude under the color-factor shift implies the following condition on the integrands

$$
0=\int \frac{d^{D} \ell}{(2 \pi)^{D}}\left[k_{4} \cdot \ell I(1,2,3,4)+k_{4} \cdot\left(\ell+k_{1}\right) I(1,4,2,3)+k_{4} \cdot\left(\ell+k_{1}+k_{2}\right) I(1,2,4,3)\right] .
$$

Relations of this form were first uncovered in refs. [49-51] from the perspective of on-shell recursion relations, and revisited recently using monodromy relations in string theory [75]. (See also ref. [76] for BCJ-type relations among loop-level integrands.) Conversely, if the integrands of the one-loop amplitude of a theory can be shown to satisfy eq. (9.16), then it follows that the one-loop amplitude is invariant under the color-factor shift, avoiding the need to invoke color-kinematic duality.

It is straightforward to generalize these considerations to one-loop $n$-gluon amplitudes. We may define a color-factor shift associated with any external gluon $a$, but for simplicity of presentation we will focus on gluon $n$. Let $\sigma$ denote a permutation of $\{2, \cdots, n-1\}$. The color factor $c_{\ldots[}[(b) n] \ldots$ shown in figure 6 undergoes a shift

$$
\delta_{n} c_{\ldots[\sigma(b) n] \ldots} \propto k_{n} \cdot k_{\sigma(b)}
$$

because gluon $n$ is attached to gluon leg $\sigma(b)$. Requiring the shifts to respect the Jacobi relation (9.8) implies

$$
\delta_{n} c_{\ldots \sigma(b) n \sigma(b+1) \cdots}=\delta_{n} c_{\ldots \sigma(b-1) n \sigma(b) \cdots}+\delta_{n} c_{\ldots[\sigma(b) n] \cdots}
$$

We must additionally define the shifts

$$
\delta_{n} c_{1 \sigma(2) \cdots \sigma(n-1) n}=\alpha_{n, \sigma} k_{n} \cdot \ell
$$

for a set of half ${ }^{16}$ of the permutations $\sigma$, where $\alpha_{n, \sigma}$ are a set of $(n-2) ! / 2$ independent arbitrary constants. Together these conditions imply that the shifts of the ring color factors are given by

$$
\delta_{n} c_{1 \sigma(2) \cdots \sigma(b-1) n \sigma(b) \cdots \sigma(n)}=\alpha_{n, \sigma}\left(k_{n} \cdot \ell+k_{n} \cdot k_{1}+\sum_{c=2}^{b-1} k_{n} \cdot k_{\sigma(c)}\right), \quad b \in\{2, \cdots, n-1\}
$$

and the shifts of all other one-loop color factors are fixed by requiring that they respect the Jacobi relations. Thus there is an $(n-2) ! / 2$-dimensional family of one-loop color-factor shifts associated with gluon $n$.

Applying the shift (9.20) to eq. (9.5), we obtain

$$
\delta_{n} \mathcal{A}_{n,(n-1) \operatorname{gon}}^{(1)} \propto \int \frac{d^{D} \ell}{(2 \pi)^{D}} \sum_{b=1}^{n-1} \frac{\left(n_{\ldots[b n] \ldots}+n_{\ldots b-1, n, b \cdots}-n_{\cdots b, n, b+1 \cdots}\right)}{\prod_{c=1}^{b}\left(\ell+\sum_{a=1}^{c-1} k_{a}\right)^{2} \prod_{d=b+1}^{n-1}\left(\ell-\sum_{a=d}^{n-1} k_{a}\right)^{2}} .
$$

\footnotetext{
${ }^{16}$ The shifts of the other half are then determined by the reflection symmetry (9.7). For example for the four-point case $\delta_{4} c_{1234}=\alpha_{4,23} k_{4} \cdot \ell$ but $\delta_{4} c_{1324}=\delta_{4} c_{1423}=\alpha_{4,23} k_{4} \cdot\left(\ell+k_{1}\right)$, which is not of the form (9.19).
} 
For $\mathcal{N}=4$ supersymmetric Yang-Mills theory, numerators for the one-loop five-gluon [10] and higher-point $[13,16]$ amplitudes have been constructed which satisfy color-kinematic duality

$$
0=n_{\ldots[b n] \cdots}+n_{\cdots b-1, n, b \cdots}-n_{\cdots b, n, b+1 \cdots}
$$

and which therefore imply that eq. (9.21) vanishes. The shifts of the terms in the cubic vertex expansion obtained from other $(n-1)$-point diagrams $I$ similarly vanish. Thus, we have established that these amplitudes possess one-loop color-factor symmetry. The oneloop $n$-point amplitudes of the bi-adjoint scalar theory also possess this symmetry because the second copy of the color factors $\tilde{c}_{i}$ obey Jacobi identities (9.8).

As we did above for the four-gluon amplitude, we can use this invariance to derive constraints on the integrands of color-ordered amplitudes. Eq. (9.14) can be rewritten as

$\mathcal{A}_{n}^{(1)}=\sum_{\sigma \in S_{n-2} / \mathbb{Z}_{2}} \int \frac{d^{D} \ell}{(2 \pi)^{D}} \sum_{b=2}^{n} c_{1 \sigma(2) \cdots \sigma(b-1) n \sigma(b) \cdots \sigma(n-1)} I(1, \sigma(2), \cdots, \sigma(b-1), n, \sigma(b), \cdots, \sigma(n-1))$

Invariance of this expression under eq. (9.20) together the independence of the parameters $\alpha_{n, \sigma}$ yields

$$
0=\int \frac{d^{D} \ell}{(2 \pi)^{D}} \sum_{b=2}^{n} k_{n} \cdot\left(\ell+k_{1}+\sum_{c=2}^{b-1} k_{\sigma(c)}\right) I(1, \sigma(2), \cdots, \sigma(b-1), n, \sigma(b), \cdots, \sigma(n-1))
$$

the relations uncovered in refs. [49-51, 75]. Conversely, if we were to establish that the integrands of the color-ordered amplitudes of a given theory satisfy eq. (9.24), we would have proven that the one-loop amplitude is invariant under the color-factor shift, independently of the assumption of color-kinematic duality. Further study of this alternate path is merited.

\section{Discussion and conclusions}

In this paper, we have introduced a new set of symmetries of gauge-theory amplitudes, which act as momentum-dependent shifts on the color factors appearing in the cubic decomposition of the amplitude. These symmetries are intimately linked to the presence of massless gauge bosons in the amplitude (or massless adjoint scalars in the case of the bi-adjoint scalar theory) and can be considered generalizations of the radiation symmetry of ref. [36]. We demonstrated that a wide class of tree-level gauge-theory amplitudes are invariant under these shifts, using a representation of the amplitude known as the radiation vertex expansion [35]. We also introduced a related but distinct cubic vertex expansion of the amplitude, and used this to derive a set of generalized-gauge-invariant constraints on the kinematic numerators appearing in the cubic decomposition of the amplitude. All known BCJ relations for tree-level gauge-theory amplitudes [1, 4, 5, 41, 42] follow as a direct consequence of the color-factor symmetry (this paper and ref. [47]). Finally, we generalized the cubic vertex expansion and color-factor symmetry to loop level. We showed 
that one-loop amplitudes that satisfy color-kinematic duality are invariant under the oneloop color-factor symmetry, and derived a set of relations among the integrands of one-loop color-ordered amplitudes.

Let us take a look at the connection between the color-factor symmetry and more fundamental symmetries of the Lagrangian, gauge and Poincaré invariance [35]. The color-factor symmetry follows as a result of the vanishing of certain expressions, namely eqs. (4.19), (7.10), and (7.22), associated with the cubic vertices of a gauge-theory amplitude, and eqs. (4.23), (4.24), and (7.27), associated with the quartic vertices. It is illustrative to examine the various contributions in a soft expansion in the gluon momentum $k_{a}$, even though the color-factor symmetry is exact in $k_{a}$.

The leading term in the soft expansion corresponds to the $\mathcal{O}\left(k_{a}^{0}\right)$ term in each of eqs. (4.19), (4.23), (7.10), (7.22), and (7.27). These are all proportional to $\sum_{r} \varepsilon_{a} \cdot K_{r}$ where $K_{r}$ are the momenta flowing out of each leg of the vertex. This vanishes by $\varepsilon_{a} \cdot k_{a}=0$ together with momentum conservation $k_{a}+\sum_{r} K_{r}=0$ - a result of symmetry under spacetime translations.

The subleading term in the soft expansion corresponds to the $\mathcal{O}\left(k_{a}^{1}\right)$ term in each of eqs. (4.19), (7.10), (7.22), and (7.27), and to eq. (4.24). These expressions are all given by a sum of angular momentum generators $J_{r}^{\alpha \beta}$, which act as a first-order Lorentz transformation on the relevant vertex factors. They vanish by Lorentz invariance.

Thus the first two terms in the soft expansion vanish as a result of Poincaré invariance. It is a little more difficult to pin down the underlying symmetry responsible for the vanishing of the sub-subleading terms in eq. (4.19) and (7.22), together with an analogous expression for spin-one particles. The $\mathcal{O}\left(k_{a}^{2}\right)$ term in eq. (4.19) is proportional to

$$
\begin{aligned}
& \left(S_{1}^{\alpha \beta}\right)_{\nu}^{\mu_{1}} \frac{\partial}{\partial K_{1 \gamma}} V^{\nu \mu_{2} \mu_{3}}\left(K_{1}, K_{2}, K_{3}\right)+\left(S_{2}^{\alpha \beta}\right)^{\mu_{2}} \frac{\partial}{\partial K_{2 \gamma}} V^{\mu_{1} \nu \mu_{3}}\left(K_{1}, K_{2}, K_{3}\right)+\left(S_{3}^{\alpha \beta}\right)^{\mu_{3}} \frac{\partial}{\partial K_{3 \gamma}} V^{\mu_{1} \mu_{2} \nu}\left(K_{1}, K_{2}, K_{3}\right) \\
& =2 i\left(-\eta^{\alpha \mu_{1}} \eta^{\beta \mu_{2}} \eta^{\gamma \mu_{3}}+\eta^{\alpha \mu_{1}} \eta^{\gamma \mu_{2}} \eta^{\beta \mu_{3}}\right)+(\text { cyclic permutations of 123) }
\end{aligned}
$$

and the $\mathcal{O}\left(k_{a}^{2}\right)$ term in eq. (7.22) is proportional to

$$
\left(S_{3}^{\alpha \beta}\right)^{\mu_{3}} \frac{\partial}{\partial K_{3 \gamma}} V^{\nu}\left(K_{1}, K_{2}, K_{3}\right)=\lambda\left(\eta^{\alpha \mu_{3}} \eta^{\beta \gamma}-\eta^{\beta \mu_{3}} \eta^{\alpha \gamma}\right) .
$$

Neither expression vanishes by itself but both do when contracted with $\varepsilon_{a \alpha} k_{a \beta} k_{a \gamma}$ for gluon $a$. These identities, which go beyond the first-order Poincaré cancellation and are connected to Yang-Mills gauge symmetry, are key ingredients contributing to the colorfactor symmetry.

Returning to our discussion in the introduction of the connection of the color-factor symmetry with the photon radiation symmetry and radiation zeros (for a collection of early references, see refs. [23, 24, 35-38, 77-81]), we have uncovered some additional analogs. For example, we can write a factorized form for the four-gluon amplitude (2.1)

$$
\mathcal{A}_{4}=s\left(\frac{c_{s}}{s}-\frac{c_{t}}{t}\right)\left(\frac{n_{s}}{s}-\frac{n_{u}}{u}\right)
$$

which vanishes at $c_{s} / s=c_{t} / t=c_{u} / u=$ const. This is a non-abelian version of the original radiation zero studied almost forty years ago in $q \bar{q} W \gamma$ and $e \nu W \gamma$ reactions, with a zero at 
$Q_{c} / k_{c} \cdot k_{a}=$ constant. This original radiation factorization and its zero led to the prediction of a measurable experimental dip, which has now been confirmed $[82,83]$. The analogous zero in the four-gluon amplitude is washed out, however, by the color averaging that must be performed in observable quark-gluon processes.

In the generalization to tree-level $n$-point amplitudes, the abelian radiation symmetry and existence of zeros for $Q_{c} / k_{c} \cdot k_{a}=$ constant (for photon momentum $k_{a}$ ) rest on having gauge-theory couplings, as noted earlier. The non-abelian color-factor symmetry uncovered in this paper can also lead to zeros in $n$-point amplitudes, but with an important complication. The invariance under $c_{i} \rightarrow c_{i}+\alpha_{i} \sum_{c \in S_{a, i}} k_{c} \cdot k_{a}$ for the attachment of a gluon with momentum $k_{a}$ cannot be used to systematically cancel out the complete $n$-gluon amplitude using an overall common value for $\alpha_{i}$. Because of the Jacobi relations, that overall common value must vanish. There are in principle zeros, however, for separate islands of $\alpha_{i}$ values. Although they are again washed out by color-averaging, the generalized factorization coming from the color-factor symmetry remains useful for theoretical analysis of tree amplitudes. In a different direction, note that the BCJ form of the gluon amplitudes has been utilized in the planar zeros studied recently in refs. [84, 85].

The analogs described above are a bridge to a final overall remark. It has been helpful to think of gluon emission or absorption as effecting a (first-order) transformation in both color and kinematic space simultaneously on the graph to which it is attached. In particular, the attachments lead to transformations of the various legs and vertices of the "parent" diagram in either momentum or space-time representations. All the parent wave functions end up transformed, and identities derived from eq. (2.20) for the different spins, e.g., eq. (7.5) and (7.32), yield exactly the two terms expected from the propagator with its bilinearity in the wave functions. The cancellations highlighted throughout this paper arise precisely because we consider those theories whose amplitudes transform covariantly under color and kinematic transformations. Adding all possible massless gluon attachments to a complete set of parent graphs leads to a sum of corresponding color shifts that vanishes because of invariance. Such a picture should help in finding directions in the diagrammatic analyses of a variety of extensions of the gauge theories considered in this paper, supersymmetric and otherwise.

\section{Acknowledgments}

This material is based upon work supported by the National Science Foundation under Grants Nos. PHY14-16123 and PFI:BIC 1318206. RWB is also supported by funds made available through a CWRU Institute Professorship Chair. SGN gratefully acknowledges sabbatical support from the Simons Foundation (Grant No. 342554 to Stephen Naculich). He would also like to thank the Michigan Center for Theoretical Physics and the Physics Department of the University of Michigan for generous hospitality and for providing a welcoming and stimulating sabbatical environment. 


\section{A Five-gluon amplitudes}

In this appendix, we use the five-gluon amplitude to provide an explicit example of the cubic vertex expansion (2.21) introduced in section 2 , and the relations among kinematic numerators (2.26) resulting from the color-factor symmetry.

The cubic decomposition (2.8) of the five-gluon amplitude is given by

$$
\begin{aligned}
\mathcal{A}_{5}= & \frac{\mathbf{c}_{12345} n_{12345}}{s_{12} s_{45}}+\frac{\mathbf{c}_{32145} n_{32145}}{s_{23} s_{45}}+\frac{\mathbf{c}_{13245} n_{13245}}{s_{13} s_{45}}+\frac{\mathbf{c}_{13425} n_{13425}}{s_{13} s_{25}}+\frac{\mathbf{c}_{13524} n_{13524}}{s_{13} s_{24}} \\
& +\frac{\mathbf{c}_{12435} n_{12435}}{s_{12} s_{35}}+\frac{\mathbf{c}_{42135} n_{42135}}{s_{24} s_{35}}+\frac{\mathbf{c}_{14235} n_{14235}}{s_{14} s_{35}}+\frac{\mathbf{c}_{14325} n_{14325}}{s_{14} s_{25}}+\frac{\mathbf{c}_{14523} n_{14523}}{s_{14} s_{23}} \\
& +\frac{\mathbf{c}_{42315} n_{42315}}{s_{24} s_{15}}+\frac{\mathbf{c}_{32415} n_{32415}}{s_{23} s_{15}}+\frac{\mathbf{c}_{34215} n_{34215}}{s_{34} s_{15}}+\frac{\mathbf{c}_{34125} n_{34125}}{s_{34} s_{25}}+\frac{\mathbf{c}_{34512} n_{34512}}{s_{34} s_{12}}
\end{aligned}
$$

where $\mathbf{c}_{\alpha}$ are half-ladder color factors defined in eq. (2.9). Let us recast this amplitude in a cubic vertex expansion with respect to gluon 2 . We have already arranged the terms in eq. (A.1) so that each line corresponds to one of the four-gluon diagrams $I$ obtained by omitting gluon 2. We rewrite the denominator of the third term of the first line as

$$
\frac{1}{s_{13} s_{45}}=\frac{1}{s_{45}\left(-s_{12}-s_{23}\right)}+\frac{1}{s_{13}\left(-s_{24}-s_{25}\right)}
$$

and similarly the denominators of the third terms of the other two lines to obtain

$$
\begin{aligned}
\mathcal{A}_{5}= & \frac{1}{s_{45}}\left(\frac{\mathbf{c}_{12345} n_{12345}}{s_{12}}+\frac{\mathbf{c}_{32145} n_{32145}}{s_{23}}-\frac{\mathbf{c}_{13245} n_{13245}}{s_{12}+s_{23}}\right) \\
& +\frac{1}{s_{13}}\left(-\frac{\mathbf{c}_{13245} n_{13245}}{s_{24}+s_{25}}+\frac{\mathbf{c}_{13425} n_{13425}}{s_{25}}+\frac{\mathbf{c}_{13524} n_{13524}}{s_{24}}\right) \\
& +\frac{1}{s_{35}}\left(\frac{\mathbf{c}_{12435} n_{12435}}{s_{12}}+\frac{\mathbf{c}_{42135} n_{42135}}{s_{24}}-\frac{\mathbf{c}_{14235} n_{14235}}{s_{12}+s_{24}}\right) \\
& +\frac{1}{s_{14}}\left(-\frac{\mathbf{c}_{14235} n_{14235}}{s_{23}+s_{25}}+\frac{\mathbf{c}_{14325} n_{14325}}{s_{25}}+\frac{\mathbf{c}_{14523} n_{14523}}{s_{23}}\right) \\
& +\frac{1}{s_{15}}\left(\frac{\mathbf{c}_{42315} n_{42315}}{s_{24}}+\frac{\mathbf{c}_{32415} n_{32415}}{s_{23}}-\frac{\mathbf{c}_{34215} n_{34215}}{s_{23}+s_{24}}\right) \\
& +\frac{1}{s_{34}}\left(-\frac{\mathbf{c}_{34215} n_{34215}}{s_{12}+s_{25}}+\frac{\mathbf{c}_{34125} n_{34125}}{s_{25}}+\frac{\mathbf{c}_{34512} n_{34512}}{s_{12}}\right)
\end{aligned}
$$

which is precisely of the form of the cubic vertex expansion (2.21). To make this connection more explicit, note that the first two lines of eq. (A.3) correspond to adding gluon 2 to the four-gluon diagram shown in figure 7 which we label as $I=1$. The color factors $c_{(a, I, v, r)}$ (see figure 1) associated with the left- and right-hand vertices of this diagram are

$$
\begin{array}{lll}
c_{(2,1, L, 1)}=\mathbf{c}_{12345} & c_{(2,1, L, 2)}=-\mathbf{c}_{32145} & c_{(2,1, L, 3)}=-\mathbf{c}_{13245} \\
c_{(2,1, R, 1)}=\mathbf{c}_{13524} & c_{(2,1, R, 2)}=-\mathbf{c}_{13425} & c_{(2,1, R, 3)}=\mathbf{c}_{13245}
\end{array}
$$

and obey $\sum_{r=1}^{3} c_{(a, I, v, r)}=0$. The relative signs result from flipping legs across lines. Because $\mathbf{c}_{13245}$ is associated with both left- and right-hand vertices, we have $c_{(2,1, L, 3)}=$ 


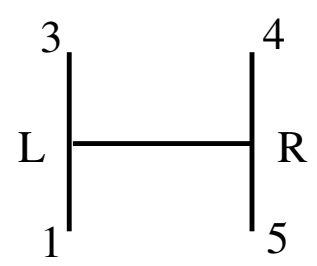

Figure 7. One of the four-gluon diagrams to which gluon 2 is added in all possible ways.

$-c_{(2,1, R, 3)}$. Since $K_{(2,1, L, 3)}=-K_{(2,1, R, 3)}$, this implies that $\alpha_{(2, I, L)}=\alpha_{(2, I, R)}$ as discussed in subsection 2.4.

The six independent five-gluon half-ladder color factors (in the notation of ref. [1]) are

$$
\begin{array}{lll}
c_{1}=\mathbf{c}_{12345} & c_{15}=\mathbf{c}_{13245} & c_{9}=\mathbf{c}_{13425} \\
c_{12}=\mathbf{c}_{12435} & c_{14}=\mathbf{c}_{14235} & c_{6}=\mathbf{c}_{14325} .
\end{array}
$$

According to eq. (2.14), the color-factor shifts associated with gluon 2 act as

$$
\begin{array}{lll}
\delta_{2} c_{1}=\alpha s_{12} & \delta_{2} c_{15}=\alpha\left(s_{12}+s_{23}\right) & \delta_{2} c_{9}=-\alpha s_{25} \\
\delta_{2} c_{12}=\alpha^{\prime} s_{12} & \delta_{2} c_{14}=\alpha^{\prime}\left(s_{12}+s_{24}\right) & \delta_{2} c_{6}=-\alpha^{\prime} s_{25}
\end{array}
$$

where $\alpha=\alpha_{2,34}$ and $\alpha^{\prime}=\alpha_{2,43}$ are arbitrary constants. The nine remaining five-gluon color factors, and the action of the color-factor shifts thereon, are determined by the Jacobi relations to be

$$
\begin{array}{rlrl}
c_{2} & =\mathbf{c}_{23451}=c_{1}+c_{6}-c_{14}-c_{15}, & & \delta_{2} c_{2}=\left(\alpha^{\prime}-\alpha\right) s_{23} \\
c_{3} & =\mathbf{c}_{34512}=c_{1}-c_{12}, & \delta_{2} c_{3}=\left(\alpha-\alpha^{\prime}\right) s_{12} \\
c_{4} & =\mathbf{c}_{45123}=c_{1}-c_{15}, & \delta_{2} c_{4}=-\alpha s_{23} \\
c_{5} & =\mathbf{c}_{51234}=c_{1}+c_{6}-c_{9}-c_{12}, & & \delta_{2} c_{5}=\left(\alpha-\alpha^{\prime}\right)\left(s_{12}+s_{25}\right) \\
c_{7} & =\mathbf{c}_{32514}=c_{6}-c_{14}, & \delta_{2} c_{7}=\alpha^{\prime} s_{23} \\
c_{8} & =\mathbf{c}_{25143}=c_{6}-c_{9}, & \delta_{2} c_{8}=\left(\alpha-\alpha^{\prime}\right) s_{25} \\
c_{10} & =\mathbf{c}_{42513}=c_{9}-c_{15}, & \delta_{2} c_{10}=\alpha s_{24} \\
c_{11} & =\mathbf{c}_{51342}=c_{9}+c_{12}-c_{14}-c_{15}, & \delta_{2} c_{11}=\left(\alpha-\alpha^{\prime}\right) s_{24} \\
c_{13} & =\mathbf{c}_{35124}=c_{12}-c_{14}, & \delta_{2} c_{13}=-\alpha^{\prime} s_{24} .
\end{array}
$$

Applying this shift to eq. (A.3), we obtain

$$
\begin{aligned}
\delta_{2} \mathcal{A}_{5}= & \alpha_{2,34}\left(\frac{n_{1}-n_{4}-n_{15}}{s_{45}}+\frac{n_{15}-n_{9}+n_{10}}{s_{13}}+\frac{n_{11}-n_{2}+n_{5}}{s_{15}}+\frac{-n_{5}+n_{8}+n_{3}}{s_{34}}\right) \\
& +\alpha_{2,43}\left(\frac{n_{12}-n_{13}-n_{14}}{s_{35}}+\frac{n_{14}-n_{6}+n_{7}}{s_{14}}+\frac{-n_{11}+n_{2}-n_{5}}{s_{15}}+\frac{n_{5}-n_{8}-n_{3}}{s_{34}}\right)=0
\end{aligned}
$$

which is precisely of the form of eq. (2.26). The color-factor shift with respect to gluon 3 instead yields 


$$
\begin{aligned}
\delta_{3} \mathcal{A}_{5}= & \alpha_{3,24}\left(\frac{n_{1}-n_{4}-n_{15}}{s_{45}}+\frac{n_{3}-n_{1}+n_{12}}{s_{12}}+\frac{n_{11}-n_{2}+n_{5}}{s_{15}}+\frac{n_{10}-n_{11}+n_{13}}{s_{24}}\right) \\
& +\alpha_{3,42}\left(\frac{n_{6}-n_{8}-n_{9}}{s_{25}}+\frac{n_{14}-n_{6}+n_{7}}{s_{14}}+\frac{-n_{11}+n_{2}-n_{5}}{s_{15}}+\frac{-n_{10}+n_{11}-n_{13}}{s_{24}}\right)=0 .
\end{aligned}
$$

Since $\alpha_{2,34}, \alpha_{2,43}, \alpha_{3,24}$, and $\alpha_{3,42}$ are independent arbitrary constants, each expression in parentheses vanishes, yielding four independent constraint equations on the kinematic numerators of the five-gluon amplitude. No additional independent constraints are obtained from the color-factor symmetries associated with the other three gluons. These "generalized Jacobi relations" for five-gluon amplitudes were previously derived in refs. [39, 40] by using the properties of string theory amplitudes.

Open Access. This article is distributed under the terms of the Creative Commons Attribution License (CC-BY 4.0), which permits any use, distribution and reproduction in any medium, provided the original author(s) and source are credited.

\section{References}

[1] Z. Bern, J.J.M. Carrasco and H. Johansson, New relations for gauge-theory amplitudes, Phys. Rev. D 78 (2008) 085011 [arXiv:0805.3993] [INSPIRE].

[2] Z. Bern, J.J.M. Carrasco and H. Johansson, Perturbative quantum gravity as a double copy of gauge theory, Phys. Rev. Lett. 105 (2010) 061602 [arXiv: 1004.0476] [INSPIRE].

[3] Z. Bern, T. Dennen, Y.-T. Huang and M. Kiermaier, Gravity as the square of gauge theory, Phys. Rev. D 82 (2010) 065003 [arXiv: 1004.0693] [INSPIRE].

[4] N.E.J. Bjerrum-Bohr, P.H. Damgaard and P. Vanhove, Minimal basis for gauge theory amplitudes, Phys. Rev. Lett. 103 (2009) 161602 [arXiv:0907.1425] [INSPIRE].

[5] B. Feng, R. Huang and Y. Jia, Gauge amplitude identities by on-shell recursion relation in S-matrix program, Phys. Lett. B 695 (2011) 350 [arXiv: 1004.3417] [InSPIRE].

[6] T. Sondergaard, Perturbative gravity and gauge theory relations: a review, Adv. High Energy Phys. 2012 (2012) 726030 [arXiv:1106.0033] [INSPIRE].

[7] D. Vaman and Y.-P. Yao, Constraints and generalized gauge transformations on tree-level gluon and graviton amplitudes, JHEP 11 (2010) 028 [arXiv:1007.3475] [INSPIRE].

[8] S. Stieberger, Open \& closed vs. pure open string disk amplitudes, arXiv:0907.2211 [INSPIRE].

[9] Y.-X. Chen, Y.-J. Du and B. Feng, A proof of the explicit minimal-basis expansion of tree amplitudes in gauge field theory, JHEP 02 (2011) 112 [arXiv:1101.0009] [INSPIRE].

[10] J.J. Carrasco and H. Johansson, Five-point amplitudes in $N=4$ super-Yang-Mills theory and $N=8$ supergravity, Phys. Rev. D 85 (2012) 025006 [arXiv:1106.4711] [InSPIRE].

[11] Z. Bern, C. Boucher-Veronneau and H. Johansson, $N \geq 4$ supergravity amplitudes from gauge theory at one loop, Phys. Rev. D 84 (2011) 105035 [arXiv:1107.1935] [INSPIRE].

[12] Z. Bern, J.J.M. Carrasco, L.J. Dixon, H. Johansson and R. Roiban, Simplifying multiloop integrands and ultraviolet divergences of gauge theory and gravity amplitudes, Phys. Rev. D 85 (2012) 105014 [arXiv: 1201.5366] [INSPIRE]. 
[13] E.Y. Yuan, Virtual color-kinematics duality: 6-pt 1-loop MHV amplitudes, JHEP 05 (2013) 070 [arXiv: 1210.1816] [INSPIRE].

[14] R.H. Boels, B.A. Kniehl, O.V. Tarasov and G. Yang, Color-kinematic duality for form factors, JHEP 02 (2013) 063 [arXiv:1211.7028] [INSPIRE].

[15] J.J.M. Carrasco, M. Chiodaroli, M. Günaydin and R. Roiban, One-loop four-point amplitudes in pure and matter-coupled $N \leq 4$ supergravity, JHEP 03 (2013) 056 [arXiv: 1212.1146] [INSPIRE].

[16] N.E.J. Bjerrum-Bohr, T. Dennen, R. Monteiro and D. O'Connell, Integrand oxidation and one-loop colour-dual numerators in $N=4$ gauge theory, JHEP 07 (2013) 092 [arXiv: 1303.2913] [INSPIRE].

[17] Z. Bern, S. Davies, T. Dennen, A.V. Smirnov and V.A. Smirnov, Ultraviolet properties of $N=4$ supergravity at four loops, Phys. Rev. Lett. 111 (2013) 231302 [arXiv:1309.2498] [INSPIRE].

[18] Z. Bern, S. Davies and T. Dennen, Enhanced ultraviolet cancellations in $N=5$ supergravity at four loops, Phys. Rev. D 90 (2014) 105011 [arXiv: 1409.3089] [InSPIRE].

[19] Z. Bern, S. Davies, T. Dennen, Y.-T. Huang and J. Nohle, Color-kinematics duality for pure Yang-Mills and gravity at one and two loops, Phys. Rev. D 92 (2015) 045041 [arXiv: 1303.6605] [INSPIRE].

[20] J. Nohle, Color-kinematics duality in one-loop four-gluon amplitudes with matter, Phys. Rev. D 90 (2014) 025020 [arXiv:1309.7416] [INSPIRE].

[21] J.J.M. Carrasco, Gauge and gravity amplitude relations, arXiv:1506.00974 [INSPIRE].

[22] C.R. Mafra, O. Schlotterer and S. Stieberger, Explicit BCJ numerators from pure spinors, JHEP 07 (2011) 092 [arXiv: 1104.5224] [INSPIRE].

[23] D.-P. Zhu, Zeros in scattering amplitudes and the structure of non-Abelian gauge theories, Phys. Rev. D 22 (1980) 2266 [INSPIRE].

[24] C.J. Goebel, F. Halzen and J.P. Leveille, Angular zeros of Brown, Mikaelian, Sahdev and Samuel and the factorization of tree amplitudes in gauge theories, Phys. Rev. D 23 (1981) 2682 [INSPIRE].

[25] R. Monteiro and D. O'Connell, The kinematic algebra from the self-dual sector, JHEP 07 (2011) 007 [arXiv: 1105.2565] [INSPIRE].

[26] N.E.J. Bjerrum-Bohr, P.H. Damgaard, R. Monteiro and D. O'Connell, Algebras for amplitudes, JHEP 06 (2012) 061 [arXiv: 1203.0944] [INSPIRE].

[27] C.-H. Fu, Y.-J. Du and B. Feng, An algebraic approach to BCJ numerators, JHEP 03 (2013) 050 [arXiv: 1212.6168] [INSPIRE].

[28] R.H. Boels, R.S. Isermann, R. Monteiro and D. O'Connell, Colour-kinematics duality for one-loop rational amplitudes, JHEP 04 (2013) 107 [arXiv: 1301.4165] [INSPIRE].

[29] M. Tolotti and S. Weinzierl, Construction of an effective Yang-Mills Lagrangian with manifest BCJ duality, JHEP 07 (2013) 111 [arXiv:1306.2975] [INSPIRE].

[30] D. Vaman and Y.-P. Yao, Color kinematic symmetric (BCJ) numerators in a light-like gauge, JHEP 12 (2014) 036 [arXiv:1408.2818] [INSPIRE].

[31] P. Mastrolia, A. Primo, U. Schubert and W.J. Torres Bobadilla, Off-shell currents and color-kinematics duality, Phys. Lett. B 753 (2016) 242 [arXiv: 1507.07532] [INSPIRE]. 
[32] S. Lee, C.R. Mafra and O. Schlotterer, Non-linear gauge transformations in D $=10 S Y M$ theory and the BCJ duality, JHEP 03 (2016) 090 [arXiv: 1510.08843] [INSPIRE].

[33] C.R. Mafra and O. Schlotterer, Berends-Giele recursions and the BCJ duality in superspace and components, JHEP 03 (2016) 097 [arXiv: 1510.08846] [INSPIRE].

[34] C.-H. Fu and K. Krasnov, Colour-kinematics duality and the Drinfeld double of the Lie algebra of diffeomorphisms, arXiv:1603.02033 [INSPIRE].

[35] R.W. Brown, K.L. Kowalski and S.J. Brodsky, Classical radiation zeros in gauge theory amplitudes, Phys. Rev. D 28 (1983) 624 [inSPIRE].

[36] R.W. Brown, Classical radiation symmetry in gauge theories, in Europhysics Study Conference: Electroweak Effects at High Energies, Erice Italy February 1-15 1983 [INSPIRE].

[37] S.J. Brodsky and R.W. Brown, Zeros in amplitudes: gauge theory and radiation interference, Phys. Rev. Lett. 49 (1982) 966 [inSPIRE].

[38] R.W. Brown and K.L. Kowalski, Classical radiation zeros addendum: spin dependent null zone, Phys. Rev. D 29 (1984) 2100 [InSPIRE].

[39] N.E.J. Bjerrum-Bohr, P.H. Damgaard, T. Sondergaard and P. Vanhove, Monodromy and Jacobi-like relations for color-ordered amplitudes, JHEP 06 (2010) 003 [arXiv:1003.2403] [INSPIRE].

[40] S.H. Henry Tye and Y. Zhang, Dual identities inside the gluon and the graviton scattering amplitudes, JHEP 06 (2010) 071 [Erratum ibid. 04 (2011) 114] [arXiv:1003.1732] [INSPIRE].

[41] S.G. Naculich, Scattering equations and BCJ relations for gauge and gravitational amplitudes with massive scalar particles, JHEP 09 (2014) 029 [arXiv:1407.7836] [INSPIRE].

[42] H. Johansson and A. Ochirov, Color-kinematics duality for QCD amplitudes, JHEP 01 (2016) 170 [arXiv: 1507.00332] [INSPIRE].

[43] T. Melia, Dyck words and multiquark primitive amplitudes, Phys. Rev. D 88 (2013) 014020 [arXiv: 1304.7809] [INSPIRE].

[44] T. Melia, Getting more flavor out of one-flavor QCD, Phys. Rev. D 89 (2014) 074012 [arXiv: 1312.0599] [INSPIRE].

[45] T. Melia, Proof of a new colour decomposition for QCD amplitudes, JHEP 12 (2015) 107 [arXiv: 1509.03297] [INSPIRE].

[46] L. de la Cruz, A. Kniss and S. Weinzierl, Proof of the fundamental BCJ relations for $Q C D$ amplitudes, JHEP 09 (2015) 197 [arXiv: 1508.01432] [INSPIRE].

[47] R.W. Brown and S.G. Naculich, Color-factor symmetry and BCJ relations for QCD amplitudes, arXiv: 1608.05291 [INSPIRE].

[48] F. Cachazo, S. He and E.Y. Yuan, Scattering of massless particles: scalars, gluons and gravitons, JHEP 07 (2014) 033 [arXiv: 1309.0885] [INSPIRE].

[49] R.H. Boels and R.S. Isermann, New relations for scattering amplitudes in Yang-Mills theory at loop level, Phys. Rev. D 85 (2012) 021701 [arXiv:1109.5888] [InSPIRE].

[50] R.H. Boels and R.S. Isermann, Yang-Mills amplitude relations at loop level from non-adjacent BCFW shifts, JHEP 03 (2012) 051 [arXiv:1110.4462] [INSPIRE]. 
[51] Y.-J. Du and H. Lüo, On general BCJ relation at one-loop level in Yang-Mills theory, JHEP 01 (2013) 129 [arXiv: 1207.4549] [INSPIRE].

[52] V. Del Duca, L.J. Dixon and F. Maltoni, New color decompositions for gauge amplitudes at tree and loop level, Nucl. Phys. B 571 (2000) 51 [hep-ph/9910563] [inSPIRE].

[53] S.G. Naculich, Scattering equations and virtuous kinematic numerators and dual-trace functions, JHEP 07 (2014) 143 [arXiv: 1404.7141] [INSPIRE].

[54] V. Del Duca, A. Frizzo and F. Maltoni, Factorization of tree QCD amplitudes in the high-energy limit and in the collinear limit, Nucl. Phys. B 568 (2000) 211 [hep-ph/9909464] [INSPIRE].

[55] R. Kleiss and H. Kuijf, Multi-gluon cross-sections and five jet production at hadron colliders, Nucl. Phys. B 312 (1989) 616 [inSPIRE].

[56] T.H. Burnett and N.M. Kroll, Extension of the low soft photon theorem, Phys. Rev. Lett. 20 (1968) 86 [INSPIRE].

[57] F.E. Low, Bremsstrahlung of very low-energy quanta in elementary particle collisions, Phys. Rev. 110 (1958) 974 [INSPIRE].

[58] V. Del Duca, High-energy bremsstrahlung theorems for soft photons, Nucl. Phys. B 345 (1990) 369 [INSPIRE].

[59] E. Laenen, G. Stavenga and C.D. White, Path integral approach to eikonal and next-to-eikonal exponentiation, JHEP 03 (2009) 054 [arXiv:0811.2067] [INSPIRE].

[60] E. Laenen, L. Magnea, G. Stavenga and C.D. White, Next-to-eikonal corrections to soft gluon radiation: a diagrammatic approach, JHEP 01 (2011) 141 [arXiv:1010.1860] [INSPIRE].

[61] E. Casali, Soft sub-leading divergences in Yang-Mills amplitudes, JHEP 08 (2014) 077 [arXiv: 1404.5551] [INSPIRE].

[62] C.D. White, Diagrammatic insights into next-to-soft corrections, Phys. Lett. B 737 (2014) 216 [arXiv: 1406.7184] [INSPIRE].

[63] Z. Bern, S. Davies, P. Di Vecchia and J. Nohle, Low-energy behavior of gluons and gravitons from gauge invariance, Phys. Rev. D 90 (2014) 084035 [arXiv: 1406.6987] [INSPIRE].

[64] J. Broedel, M. de Leeuw, J. Plefka and M. Rosso, Constraining subleading soft gluon and graviton theorems, Phys. Rev. D 90 (2014) 065024 [arXiv:1406.6574] [INSPIRE].

[65] H. Lüo, P. Mastrolia and W.J. Torres Bobadilla, Subleading soft behavior of QCD amplitudes, Phys. Rev. D 91 (2015) 065018 [arXiv:1411.1669] [INSPIRE].

[66] D. Kosower, B.-H. Lee and V.P. Nair, Multi gluon scattering: a string based calculation, Phys. Lett. B 201 (1988) 85 [INSPIRE].

[67] M.L. Mangano, The color structure of gluon emission, Nucl. Phys. B 309 (1988) 461 [INSPIRE].

[68] S.G. Naculich, CHY representations for gauge theory and gravity amplitudes with up to three massive particles, JHEP 05 (2015) 050 [arXiv: 1501.03500] [INSPIRE].

[69] Y. Geyer, L. Mason, R. Monteiro and P. Tourkine, Loop integrands for scattering amplitudes from the Riemann sphere, Phys. Rev. Lett. 115 (2015) 121603 [arXiv:1507.00321] [INSPIRE]. 
[70] C. Baadsgaard, N.E.J. Bjerrum-Bohr, J.L. Bourjaily, P.H. Damgaard and B. Feng, Integration rules for loop scattering equations, JHEP 11 (2015) 080 [arXiv:1508.03627] [INSPIRE].

[71] S. He and E.Y. Yuan, One-loop scattering equations and amplitudes from forward limit, Phys. Rev. D 92 (2015) 105004 [arXiv:1508.06027] [InSPIRE].

[72] C. Baadsgaard, N.E.J. Bjerrum-Bohr, J.L. Bourjaily, S. Caron-Huot, P.H. Damgaard and B. Feng, New representations of the perturbative S-matrix, Phys. Rev. Lett. 116 (2016) 061601 [arXiv: 1509.02169] [INSPIRE].

[73] Y. Geyer, L. Mason, R. Monteiro and P. Tourkine, One-loop amplitudes on the Riemann sphere, JHEP 03 (2016) 114 [arXiv:1511.06315] [INSPIRE].

[74] F. Cachazo, S. He and E.Y. Yuan, One-loop corrections from higher dimensional tree amplitudes, JHEP 08 (2016) 008 [arXiv:1512.05001] [INSPIRE].

[75] P. Tourkine and P. Vanhove, Higher-loop amplitude monodromy relations in string and gauge theory, arXiv:1608.01665 [INSPIRE].

[76] D. Chester, Bern-Carrasco-Johansson relations for one-loop QCD integral coefficients, Phys. Rev. D 93 (2016) 065047 [arXiv: 1601.00235] [INSPIRE].

[77] K.O. Mikaelian, Photoproduction of charged intermediate vector bosons, Phys. Rev. D 17 (1978) 750 [INSPIRE].

[78] R.W. Brown, D. Sahdev and K.O. Mikaelian, $W^{ \pm} Z^{0}$ and $W^{ \pm} \gamma$ pair production in $\nu e, p p$ and $\bar{p} p$ collisions, Phys. Rev. D 20 (1979) 1164 [InSPIRE].

[79] K.O. Mikaelian, M.A. Samuel and D. Sahdev, The magnetic moment of weak bosons

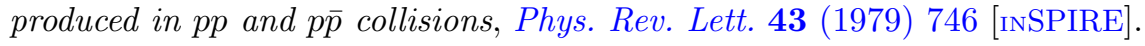

[80] M.A. Samuel, Amplitude zeros, Phys. Rev. D 27 (1983) 2724 [InSPIRE].

[81] S.G. Naculich, Physical null zones, Phys. Rev. D 28 (1983) 2297 [InSPIRE].

[82] D0 collaboration, V.M. Abazov et al., First study of the radiation-amplitude zero in $W \gamma$ production and limits on anomalous $W W \gamma$ couplings at $\sqrt{s}=1.96$ TeV, Phys. Rev. Lett. 100 (2008) 241805 [arXiv:0803.0030] [INSPIRE].

[83] CMS collaboration, Measurement of $W \gamma$ and $Z \gamma$ production in pp collisions at $\sqrt{s}=7 \mathrm{TeV}$, Phys. Lett. B 701 (2011) 535 [arXiv:1105.2758] [InSPIRE].

[84] L.A. Harland-Lang, Planar radiation zeros in five-parton QCD amplitudes, JHEP 05 (2015) 146 [arXiv: 1503.06798] [INSPIRE].

[85] D. Medrano Jimenez, A. Sabio Vera and M.A. Vazquez-Mozo, Planar zeros in gauge theories and gravity, JHEP 09 (2016) 006 [arXiv:1607.04605] [INSPIRE]. 